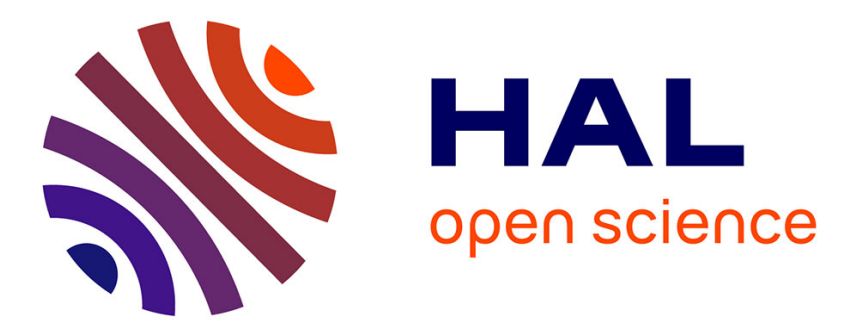

\title{
Gevrey Smoothing for Weak Solutions of the Fully Nonlinear Homogeneous Boltzmann and Kac Equations Without Cutoff for Maxwellian Molecules
}

Jean-Marie Barbaroux, Dirk Hundertmark, Tobias Ried, Semjon Vugalter

\section{- To cite this version:}

Jean-Marie Barbaroux, Dirk Hundertmark, Tobias Ried, Semjon Vugalter. Gevrey Smoothing for Weak Solutions of the Fully Nonlinear Homogeneous Boltzmann and Kac Equations Without Cutoff for Maxwellian Molecules. Archive for Rational Mechanics and Analysis, 2017, 225 (2), pp. 601-661. 10.1007/s00205-017-1101-8 . hal-01253130

\author{
HAL Id: hal-01253130 \\ https://hal.science/hal-01253130
}

Submitted on 8 Jan 2016

HAL is a multi-disciplinary open access archive for the deposit and dissemination of scientific research documents, whether they are published or not. The documents may come from teaching and research institutions in France or abroad, or from public or private research centers.
L'archive ouverte pluridisciplinaire HAL, est destinée au dépôt et à la diffusion de documents scientifiques de niveau recherche, publiés ou non, émanant des établissements d'enseignement et de recherche français ou étrangers, des laboratoires publics ou privés. 


\title{
GEVREY SMOOTHING FOR WEAK SOLUTIONS OF THE FULLY NONLINEAR HOMOGENEOUS BOLTZMANN AND KAC EQUATIONS WITHOUT CUTOFF FOR MAXWELLIAN MOLECULES
}

\author{
JEAN-MARIE BARBAROUX, DIRK HUNDERTMARK, TOBIAS RIED, AND SEMJON VUGALTER
}

\begin{abstract}
AвSTRACT. It has long been suspected that the non-cutoff Boltzmann operator has similar coercivity properties as a fractional Laplacian. This has led to the hope that the homogenous Boltzmann equation enjoys similar regularity properties as the heat equation with a fractional Laplacian. In particular, the weak solution of the fully nonlinear non-cutoff homogenous Boltzmann equation with initial datum in $L_{2}^{1}\left(\mathbb{R}^{d}\right) \cap L \log L\left(\mathbb{R}^{d}\right)$, i.e., finite mass, energy and entropy, should immediately become Gevrey regular for strictly positive times. We prove this conjecture for Maxwellian molecules.
\end{abstract}

2010 Mathematics Subject Classification: 35D10 (Primary); 35B65, 35Q20, 82B40 (Secondary) Keywords: Gevrey regularity, Non-cutoff homogeneous Boltzmann equation, Non-cutoff homogeneous Kac equation, Maxwellian molecules

\section{Contents}

1. Introduction 1

1.1. The non-cutoff Boltzmann and Kac models 2

1.2. Higher regularity of weak solutions 5

2. Gevrey regularity and (ultra-)analyticity of weak solutions with $L^{2}$ initial data 9

2.1. $L^{2}$-Reformulation of the homogeneous Boltzmann equation for weak solutions and coercivity 9

2.2. Bound on the commutation error 11

2.3. Extracting pointwise information from local $L^{2}$ bounds 16

2.4. Gevrey smoothing of weak solutions for $L^{2}$ initial data: Part I 19

2.5. Gevrey smoothing of weak solutions for $L^{2}$ initial data: Part II 23

2.6. Gevrey smoothing of weak solutions for $L^{2}$ initial data: Part III 27

3. Removing the $L^{2}$ constraint: Gevrey regularity and (ultra-)analyticity of weak solutions 32

$\begin{array}{lll}\text { Appendix A. } & L^{2} \text { type reformulation of the Boltzmann and Kac equations } & 33\end{array}$

Appendix B. $\quad H^{\infty}$ smoothing of the Boltzmann an Kac equations 36

$\begin{array}{lll}\text { Appendix C. The Kolmogorov-Landau inequality } & 38\end{array}$

Appendix D. Proof of Lemma 1.1 40

References $\quad 41$

\section{INTRODUCTION}

It has long been suspected that the non-cutoff Boltzmann operator with a singular cross section kernel has similar coercivity properties as a fractional Laplacian $(-\Delta)^{v}$, for suitable $0<v<1$. This has been made precise by Alexandre, Desvillettes, Villani, and Wennberg [3], see also the reviews by Alexandre [2] and by Villani [40] for its history, and has led to the hope that the fully nonlinear homogenous Boltzmann equation enjoys similar regularity properties as the heat equation

Date: 21 st September 2015.

(C) 2015 by the authors. Faithful reproduction of this article, in its entirety, by any means is permitted for noncommercial purposes. 
with a fractional Laplacian given by

$$
\left\{\begin{aligned}
\partial_{t} u+(-\Delta)^{v} u & =0 \\
\left.u\right|_{t=0} & =u_{0} \in L^{1}\left(\mathbb{R}^{d}\right) .
\end{aligned}\right.
$$

Using the Fourier transform one immediately sees that

$$
\widehat{u}(t, \xi)=\mathrm{e}^{-t(2 \pi|\xi|)^{2 v}} \widehat{u_{0}}(\xi) \quad \text { with } \quad \widehat{u_{0}} \in L^{\infty}\left(\mathbb{R}^{d}\right)
$$

so

$$
\sup _{t>0} \sup _{\xi \in \mathbb{R}^{d}} \mathrm{e}^{|\xi|^{2 v}}|\widehat{u}(t, \xi)| \leq\left\|u_{0}\right\|_{L^{1}\left(\mathbb{R}^{d}\right)}<\infty,
$$

that is, the Fourier transform of the solution is extremely fast decaying for strictly positive times.

Introducing the Gevrey spaces as in Definition 1.5, it is natural to expect, see, for example, Desvillettes and WennBerg [16]:

Conjecture (Gevrey smoothing). Any weak solution of the non-cutoff homogenous Boltzmann equation with a singular cross section kernel of order $v$ and with initial datum in $L_{2}^{1}\left(\mathbb{R}^{d}\right) \cap L \log L\left(\mathbb{R}^{d}\right)$, i.e., finite mass, energy and entropy, belongs to the Gevrey class $G^{\frac{1}{2 v}}\left(\mathbb{R}^{d}\right)$ for strictly positive times.

The central results of our work is a proof of this conjecture for Maxwellian molecules. In particular, we prove

Theorem. Assume that the non-cutoff Boltzman cross section has a singularity $1+2 v$ with $0<v<1$ and obeys some further technical conditions, which are true in all physically relevant cases, for details see (3) and (16). Then, for initial conditions $f_{0} \in L \log L \cap L_{m}^{1}$ with an integer

$$
m \geq \max \left(2, \frac{2^{v}-1}{2\left(2-2^{v}\right)}\right)
$$

any weak solution of the fully non-linear homogenous Boltzmann equation for Maxwellian molecules belongs to the Gevrey class $G^{\frac{1}{2 v}}$ for strictly positive times.

In particular, for $v \leq \log (9 / 5) / \log (2) \simeq 0,847996$ we have $m=2$ and the theorem does not require anything except the physically reasonable assumptions of finite mass, energy, and entropy. If $\log (9 / 5) / \log (2)<v<1$ and we assume only that $f_{0} \in L \log L \cap L_{2}^{1}$, then we prove that the solution is in $G^{\frac{\log 2}{2 \log (9 / 5)}}$, in particular, it is ultra-analytic.

(1) For a more precise formulation of our results, see Theorems 1.6, 1.8, and 1.9 for the case $m=2$ and Theorems 3.1, 3.2, and 3.3 below.

(2) We would like to stress that our results cover both the weak and strong singularity regimes, where $0<v<1 / 2$, respectively $1 / 2 \leq v<1$.

(3) The theorem above applies to all dimensions $d \geq 1$. The physical case for Maxwellian molecules in dimension $d=3$ is $v=1 / 4$.

The main problem for establishing Gevrey regularity is that, in order to use the coercivity results of Alexandre, Desvillettes, Villani and Wennberg [3], one has to bound a non-linear and nonlocal commutator of the Boltzmann kernel with certain sub-Gaussian Fourier multipliers. The main ingredient in our proof is a new way of estimating this non-local and nonlinear commutator.

1.1. The non-cutoff Boltzmann and Kac models. We study the regularity of weak solutions of the Cauchy problem

$$
\left\{\begin{array}{l}
\partial_{t} f=Q(f, f) \\
\left.f\right|_{t=0}=f_{0}
\end{array}\right.
$$

for the fully nonlinear homogeneous Boltzmann and Kac equation in $d \geq 1$ dimensions [10, 21].

For $d \geq 2$ the bilinear operator $Q$ is given by

$$
Q(g, f)=\int_{\mathbb{R}^{d}} \int_{\mathbb{S}^{d-1}} b(\cos \theta)\left(g\left(v_{*}^{\prime}\right) f\left(v^{\prime}\right)-g\left(v_{*}\right) f(v)\right) \mathrm{d} \sigma \mathrm{d} v_{*},
$$


that is, the Boltzmann collision operator for Maxwellian molecules with angular collision kernel $b$ depending only on the deviation angle $\cos \theta=\sigma \cdot \frac{v-v_{*}}{\left|v-v_{*}\right|}$ for $\sigma \in \mathbb{S}^{d-1}$. Here we use the $\sigma$ representation of the collision process, in which

$$
v^{\prime}=\frac{v+v_{*}}{2}+\frac{\left|v-v_{*}\right|}{2} \sigma, \quad v_{*}^{\prime}=\frac{v+v_{*}}{2}-\frac{\left|v-v_{*}\right|}{2} \sigma, \quad \text { for } \sigma \in \mathbb{S}^{d-1} .
$$

By symmetry properties of the Boltzmann collision operator $Q(f, f)$, the function $b$ can be assumed to be supported on angles $\theta \in\left[0, \frac{\pi}{2}\right]$, for otherwise, see [40], it can be replaced by

$$
\widetilde{b}(\cos \theta)=\left(b(\cos \theta)+b(\cos (\pi-\theta)) \mathbb{1}_{\left\{0 \leq \theta \leq \frac{\pi}{2}\right\}} .\right.
$$

We will assume that the angular collision kernel $b$ has the non-integrable singularity

$$
\sin ^{d-2} \theta b(\cos \theta) \sim \frac{\kappa}{\theta^{1+2 v}}, \quad \text { as } \theta \rightarrow 0^{+}
$$

for some $\kappa>0$ and $0<v<1$, and satisfies

$$
\int_{0}^{\pi / 2} \sin ^{d} \theta b(\cos \theta) \mathrm{d} \theta<\infty .
$$

For inverse $s$-power forces (in three spatial dimensions), described by the potential $U(r)=r^{1-s}$, $s>2$, the collision kernel is of the more general form

$$
B\left(\left|v-v_{*}\right|, \cos \theta\right)=b(\cos \theta)\left|v-v_{*}\right|^{\gamma}, \quad \gamma=\frac{s-5}{s-1},
$$

where the angular collision kernel $b$ is locally smooth with a non-integrable singularity

$$
\sin \theta b(\cos \theta) \sim K \theta^{-1-2 v}, \quad v=\frac{1}{s-1} .
$$

The case of (physical) Maxwellian molecules corresponds to the values $\gamma=0, s=5, v=\frac{1}{4}$.

For $d=1$ we set

$$
Q(g, f)=K(g, f)=\int_{\mathbb{R}} \int_{-\frac{\pi}{2}}^{\frac{\pi}{2}} b_{1}(\theta)\left(f\left(w_{*}^{\prime}\right) g\left(w^{\prime}\right)-f\left(w_{*}\right) g(w)\right) \mathrm{d} \theta \mathrm{d} w_{*},
$$

which is the Kac operator for Maxwellian molecules, and angular collision kernel $b_{1} \geq 0$. The preand post-collisional velocities are related by

$$
\left(\begin{array}{c}
w^{\prime} \\
w_{*}^{\prime}
\end{array}\right)=\left(\begin{array}{cc}
\cos \theta & -\sin \theta \\
\sin \theta & \cos \theta
\end{array}\right)\left(\begin{array}{c}
w \\
w_{*}
\end{array}\right), \quad \text { for } \theta \in\left[-\frac{\pi}{2}, \frac{\pi}{2}\right] .
$$

In the original Kac model $b_{1}$ was chosen to be constant, whereas we will assume, as in [14], that $b_{1}$ is an even function and has the non-integrable singularity

$$
b_{1}(\theta) \sim \frac{\kappa}{|\theta|^{1+2 v}}, \quad \text { for } \theta \rightarrow 0,
$$

with $0<v<1$ and some $\kappa>0$, and further satisfies

$$
\int_{-\frac{\pi}{2}}^{\frac{\pi}{2}} b_{1}(\theta) \sin ^{2} \theta \mathrm{d} \theta<\infty
$$

Making use of symmetry properties of the collision operator $K(f, f)$, we can assume $b_{1}$ to be supported on angles $\theta \in\left[-\frac{\pi}{4}, \frac{\pi}{4}\right]$, for otherwise it can be replaced by its symmetrised version

$$
\widetilde{b_{1}}(\theta)=\left(b_{1}(\theta)+b_{1}\left(\frac{\pi}{2}-\theta\right)\right) \mathbb{1}_{\left\{0 \leq \theta \leq \frac{\pi}{4}\right\}}+\left(b_{1}(\theta)+b_{1}\left(-\frac{\pi}{2}-\theta\right)\right) \mathbb{1}_{\left\{-\frac{\pi}{4} \leq \theta \leq 0\right\}} .
$$

This simple observation will be very convenient for our analysis.

We will mainly work with the weighted $L^{p}$ spaces, defined as

$$
L_{\alpha}^{p}\left(\mathbb{R}^{d}\right):=\left\{f \in L^{p}\left(\mathbb{R}^{d}\right):\langle\cdot\rangle^{\alpha} f \in L^{p}\left(\mathbb{R}^{d}\right)\right\},
$$


$p \geq 1, \alpha \in \mathbb{R}$, with norm

$$
\|f\|_{L_{\alpha}^{p}\left(\mathbb{R}^{d}\right)}=\left(\int_{\mathbb{R}^{d}}|f(v)|^{p}\langle v\rangle^{\alpha p} \mathrm{~d} v\right)^{1 / p}, \quad\langle v\rangle:=\left(1+|v|^{2}\right)^{1 / 2} .
$$

We will also use the weighted ( $L^{2}$ based) Sobolev spaces

$$
H_{\ell}^{k}\left(\mathbb{R}^{d}\right)=\left\{f \in \mathcal{S}^{\prime}\left(\mathbb{R}^{d}\right):\langle\cdot\rangle^{\ell} f \in H^{k}\left(\mathbb{R}^{d}\right)\right\}, \quad k, \ell \in \mathbb{R},
$$

where $H^{k}\left(\mathbb{R}^{d}\right)$ are the usual Sobolev spaces given by $H^{k}\left(\mathbb{R}^{d}\right)=\left\{f \in \mathcal{S}^{\prime}\left(\mathbb{R}^{d}\right):\langle\cdot\rangle^{k} \hat{f} \in L^{2}\left(\mathbb{R}^{d}\right)\right\}$, for $k \in \mathbb{R}$. The inner product on $L^{2}\left(\mathbb{R}^{d}\right)$ is given by $\langle f, g\rangle=\int_{\mathbb{R}^{d}} \overline{f(v)} g(v) \mathrm{d} v$.

It will be assumed that the initial datum $f_{0} \not \equiv 0$ is a non-negative density with finite mass, energy and entropy, which is equivalent to

$$
f_{0} \geq 0, \quad f_{0} \in L_{2}^{1}\left(\mathbb{R}^{d}\right) \cap L \log L\left(\mathbb{R}^{d}\right),
$$

where

$$
L \log L\left(\mathbb{R}^{d}\right)=\left\{f: \mathbb{R}^{d} \rightarrow \mathbb{R} \text { measurable }:\|f\|_{L \log L}=\int_{\mathbb{R}^{d}}|f(v)| \log (1+|f(v)|) \mathrm{d} v<\infty\right\},
$$

and the negative of the entropy is given by $H(f):=\int_{\mathbb{R}^{d}} f \log f \mathrm{~d} v$.

The space $L_{2}^{1}\left(\mathbb{R}^{d}\right) \cap L \log L\left(\mathbb{R}^{d}\right)$ is very natural, since

Lemma 1.1. Let $f \geq 0$. Then

$$
f \in L_{2}^{1}\left(\mathbb{R}^{d}\right) \cap L \log L\left(\mathbb{R}^{d}\right) \quad \Leftrightarrow \quad f \in L_{2}^{1}\left(\mathbb{R}^{d}\right) \text { and } H(f) \text { is finite. }
$$

We suspect that this lemma is well-known, at least to the experts, but we could not find a reference in the literature. For the reader's convenience we will give the proof in appendix D. Following is the precise definition of weak solutions which we use.

Definition 1.2 (Weak Solutions of the Cauchy Problem (1) [8, 39, 11]). Assume that the initial datum $f_{0}$ is in $L_{2}^{1}\left(\mathbb{R}^{d}\right) \cap L \log L\left(\mathbb{R}^{d}\right) . f: \mathbb{R}_{+} \times \mathbb{R}^{d} \rightarrow \mathbb{R}$ is called a weak solution to the Cauchy problem (1), if it satisfies the following conditions ${ }^{1}$ :

(i) $f \geq 0, \quad f \in \mathscr{C}\left(\mathbb{R}_{+} ; \mathscr{D}^{\prime}\left(\mathbb{R}^{d}\right)\right) \cap L^{\infty}\left(\mathbb{R}_{+} ; L_{2}^{1}\left(\mathbb{R}^{d}\right) \cap L \log L\left(\mathbb{R}^{d}\right)\right)$

(ii) $f(0, \cdot)=f_{0}$

(iii) For all $t \geq 0$, mass is conserved, $\int_{\mathbb{R}^{d}} f(t, v) \mathrm{d} v=\int_{\mathbb{R}^{d}} f_{0}(v) \mathrm{d} v$, kinetic energy is decreasing, $\int_{\mathbb{R}^{d}} f(t, v) v^{2} \mathrm{~d} v \leq \int_{\mathbb{R}^{d}} f_{0}(v) v^{2} \mathrm{~d} v$, and the entropy is increasing, $H(f(t, \cdot)) \leq H\left(f_{0}\right)$.

(iv) For all $\varphi \in \mathscr{C}^{1}\left(\mathbb{R}_{+} ; \mathscr{C}_{0}^{\infty}\left(\mathbb{R}^{d}\right)\right)$ one has

$$
\begin{gathered}
\langle f(t, \cdot), \varphi(t, v)\rangle-\left\langle f_{0}, \varphi(0, \cdot)\right\rangle-\int_{0}^{t}\left\langle f(\tau, \cdot) \partial_{\tau} \varphi(\tau, \cdot)\right\rangle \mathrm{d} \tau \\
=\int_{0}^{t}\langle Q(f, f)(\tau, \cdot), \varphi(\tau, \cdot)\rangle \mathrm{d} \tau, \quad \text { for all } t \geq 0,
\end{gathered}
$$

where the latter expression involving $Q$ is defined by

$$
\begin{aligned}
& \langle Q(f, f), \varphi\rangle \\
& \quad=\frac{1}{2} \int_{\mathbb{R}^{2 d}} \int_{\mathbb{S}^{d-1}} b\left(\frac{v-v_{*}}{\left|v-v_{*}\right|} \cdot \sigma\right) f\left(v_{*}\right) f(v)\left(\varphi\left(v^{\prime}\right)+\varphi\left(v_{*}^{\prime}\right)-\varphi(v)-\varphi\left(v_{*}\right)\right) \mathrm{d} \sigma \mathrm{d} v \mathrm{~d} v_{*},
\end{aligned}
$$

for test functions $\varphi \in W^{2, \infty}\left(\mathbb{R}^{d}\right)$ in dimension $d \geq 2$, and in one dimension

$$
\langle Q(f, f), \varphi\rangle=\langle K(f, f), \varphi\rangle=\int_{\mathbb{R}^{2}} \int_{-\frac{\pi}{4}}^{\frac{\pi}{4}} b_{1}(\theta) g\left(w_{*}\right) g(w)\left(\phi\left(w^{\prime}\right)-\phi(w)\right) \mathrm{d} \theta \mathrm{d} w \mathrm{~d} w_{*}
$$

\footnotetext{
${ }^{1}$ Throughout the text, whenever not explicitly mentioned, we will drop the dependence on $t$ of a function, i.e. $f(v):=$ $f(t, v)$ etc
} 
for test functions $\varphi \in W^{2, \infty}(\mathbb{R})$, making use of symmetry properties of the Boltzmann and Kac collision operators and cancellation effects.

Collecting results from the literature, the following is known regarding the existence, uniqueness and further properties of weak solutions.

Theorem 1.3 (Arkeryd, Desvillettes, Mischler, Goudon, Villani, Wennberg). There exists a weak solution of the Cauchy problem (1) in the sense of Definition 1.2. For $d \geq 2$ momentum and energy are conserved,

$$
\int_{\mathbb{R}^{d}} f(t, v) v \mathrm{~d} v=\int_{\mathbb{R}^{d}} f_{0}(v) v \mathrm{~d} v, \quad \int_{\mathbb{R}^{d}} f(t, v) v^{2} \mathrm{~d} v=\int_{\mathbb{R}^{d}} f_{0}(v) v^{2} \mathrm{~d} v .
$$

In the one dimensional case (Kac equation), momentum is not conserved and energy can only decrease and is conserved under the additional moment assumption $f_{0} \in L_{2 p}^{1}$ for some $p \geq 2$.

Remark 1.4. $d \geq 2$ : The existence of weak solutions of the Cauchy problem (1) with initial conditions satisfying (8) for the homogeneous Boltzmann equation was first proved by ARKERYD [7, 8] (see also the articles by Goudon [20], Villani [39], and Desvillettes [13, 14]). Uniqueness in this case was shown by Toscani and VitLani [36], see also the review articles by MischleR and WenNBerg [28] (for the cut-off case) and Desvillettes [13].

$d=1$ : For the homogeneous non-cutoff Kac equation for Maxwellian molecules existence of weak solutions was established by DesviLlettes [11].

1.2. Higher regularity of weak solutions. It has been pointed out by several authors $[2,16,40]$ that, for singular cross-sections, the Boltzmann operator essentially behaves like a singular integral operator with a leading term similar to a fractional Laplace operator $(-\Delta)^{v}$. In terms of compactness properties this has been noticed for the linearised Boltzmann kernel as early as in [33] and for the nonlinear Boltzmann kernel in [27]. Since the solutions of the heat equation with a fractional Laplacian gain a high amount of regularity for arbitrary positive times, it is natural to believe, as conjectured in [16], that weak solutions to the non-cutoff Boltzmann equation gain a certain amount of smoothness, and even analyticity, for any $t>0$. This is in sharp contrast to the fact that in the Grad's cutoff case there cannot be any smoothing effect. Instead, regularity and singularities of the initial datum get propagated in this case, see, for example, [32].

The discussion about solution of the heat equation with a fractional Laplacian motivates the following definition of Gevrey spaces, which give a convenient framework to describe this smoothing by interpolating between smooth and (ultra-)analytic functions.

Definition 1.5. Let $s>0$. A function $f$ belongs to the Gevrey class $G^{s}\left(\mathbb{R}^{d}\right)$, if there exists an $\epsilon_{0}>0$ such that

$$
e^{\epsilon_{0}\left\langle D_{v}\right\rangle^{1 / s}} f \in L^{2}\left(\mathbb{R}^{d}\right), \quad \text { where }\left\langle D_{v}\right\rangle=\left(1+\left|D_{v}\right|^{2}\right)^{1 / 2} .
$$

and we use the notation $D_{v}=-\frac{\mathrm{i}}{2 \pi} \nabla_{v}$. Thus, $G^{1}\left(\mathbb{R}^{d}\right)$ is the space of real analytic functions, and $G^{s}\left(\mathbb{R}^{d}\right)$ for $s \in(0,1)$ the space of ultra-analytic functions.

Equivalently ${ }^{2}, f \in G^{s}\left(\mathbb{R}^{d}\right)$ if $f \in \mathscr{C}^{\infty}\left(\mathbb{R}^{d}\right)$ and there exists a constant $C>0$ such that for all $k \in \mathbb{N}_{0}$ one has

$$
\left\|D^{k} f\right\|_{L^{2}\left(\mathbb{R}^{d}\right)} \leq C^{k+1}(k !)^{s},
$$

where $\left\|D^{k} f\right\|_{L^{2}}^{2}=\sup _{|\beta|=k}\left\|\partial^{\beta} f\right\|_{L^{2}}^{2}$.

The first regularisation results in this direction were due to Desvillettes for the spatially homogeneous non-cutoff Kac equation [11] and the homogeneous non-cutoff Boltzmann equation for Maxwellian molecules in two dimensions [12], where $\mathscr{C}^{\infty}$ regularisation is proved. Later, DesvILLETTES and WeNNBERg [16] proved, under rather general assumptions on the collision cross-section (excluding Maxwellian molecules, though), regularity in Schwartz space of weak solutions to the

\footnotetext{
${ }^{2}$ see, for example, Theorem 4 in [25].
} 
non-cutoff homogeneous Boltzmann equation. By quite different methods, using Littlewood-Paley decompositions, Alexandre and El Safadi [4] showed that the assumptions on the cross-section (3)(4) imply that the solutions are in $H^{\infty}$ for any positive time $t>0$. By moment propagation results for Maxwellian molecules (see TruesDell [37]) this cannot be improved to regularity in Schwartz space.

For collision cross-sections corresponding to Debye-Yukawa-type interaction potentials,

$$
\sin \theta b(\cos \theta) \sim K \theta^{-1}\left(\log \theta^{-1}\right)^{\ell} \quad \text { for } \theta \rightarrow 0 \quad \text { (with some } K>0, \ell>0 \text { ), }
$$

Morimoto, UкAI, $\mathrm{Xu}$ and Yang [30] proved the same $H^{\infty}$ regularising effect using suitable test functions in the weak formulation of the problem.

The question of the local existence of solutions in Gevrey spaces for Gevrey regular initial data with additional strong decay at infinity was first addressed in 1984 by UKAI [38], both in the spatially homogeneous and inhomogeneous setting.

We are interested in the Gevrey smoothing effect, namely that under the (physical) assumptions of finite mass, energy and entropy of the initial data, weak solutions of the homogeneous Boltzmann equation without cutoff are Gevrey functions for any strictly positive time. This question was treated in the case of the linearised Boltzmann equation in the homogeneous setting by Мовімото et al. [30], where they proved that, given $0<v<1$, weak solutions of the linearized Boltzmann equation belong to the space $G^{\frac{1}{v}}\left(\mathbb{R}^{3}\right)$ for any positive times. Still in a linearised setting, Lerner, Morimoto, Pravda-Starov and Xu [24] proved a Gelfand-Shilov smoothing effect, which includes Gevrey regularity, on radially symmetric solutions of the homogeneous non-cutoff Boltzmann equation for Maxwellian molecules. For the non-Maxwellian Boltzmann operator, Gevrey regularity was proved under very strong unphysical decay assumptions on the initial datum in [26].

For radially symmetric solutions, the homogeneous non-cutoff Boltzmann equation for Maxwellian molecules is related to the homogeneous non-cutoff Kac equation. The non-cutoff Kac equation was introduced by DesviLLETTEs in [11], where first regularity results were established, see also Desvillettes' review [14]. For this equation, the best available results so far are due to LeKRINE and $\mathrm{XU}$ [23] and Glangetas and NaJeme [19]: Lekrine and $\mathrm{XU}_{\mathrm{U}}$ [23] proved Gevrey regularisation of order $\frac{1}{2 \alpha}$ for mild singularities $0<v<\frac{1}{2}$ and all $0<\alpha<v$. Strong singularities $\frac{1}{2} \leq v<1$ were treated by Glangetas and NaJeme [19], where they prove that for $v=\frac{1}{2}$ the solution becomes Gevrey regular of order $\frac{1}{2 \alpha}$ for any $0<\alpha<\frac{1}{2}$ and Gevrey regular of order 1 , that is, analytic, when $\frac{1}{2}<v<1$. Thus, in the critical case $v=\frac{1}{2}$, the result of [19] misses analyticity of weak solutions and they do not prove ultra-analyticity in the range $0<v<1$. Moreover, both results are obtained under the additional moment assumption $f_{0} \in L_{2+2 v}^{1}(\mathbb{R})$.

Ultra-analyticity results have previously been obtained by Мовімото and $\mathrm{XU}_{\mathrm{U}}$ [31] for the homogeneous Landau equation in the Maxwellian molecules case and related simplified models in kinetic theory. The analysis of smoothing properties of Landau equation is quite different from the Boltzmann and Kac equations. The Landau equation explicitly contains a second order elliptic term, which yields coercivity, and, more importantly, certain commutators with weights in Fourier space are identically zero, which simplifies the analysis tremendously, see Proposition 2.2 in [31].

For the nonlinear non-cutoff homogeneous Boltzmann equation some partial results regarding Gevrey regularisation were obtained by Мовімото and UкаI [29] including the non-Maxwellian molecules case, but under the strong additional assumptions of Maxwellian decay and smoothness of the solution. Still with these strong decay assumptions, YIN and Zhang [42, 41] extended this result to a larger class of kinetic cross-sections.

We stress that for the main result of our paper the initial datum is only assumed to obey the natural assumptions coming from physics, i.e., finiteness of mass, energy and entropy.

Given $\beta>0$ and $\alpha \in(0,1)$ we define the Gevrey multiplier $G: \mathbb{R}_{+} \times \mathbb{R}^{d} \rightarrow \mathbb{R}$ by

$$
G(t, \eta):=e^{\beta t\langle\eta\rangle^{2 \alpha}}
$$


and for $\Lambda>0$ the cut-off Gevrey multiplier $G_{\Lambda}: \mathbb{R}_{+} \times \mathbb{R}^{d} \rightarrow \mathbb{R}$ by

$$
G_{\Lambda}(t, \eta):=G(t, \eta) \mathbb{1}_{\Lambda}(|\eta|)
$$

where $\mathbb{1}_{\Lambda}$ is the characteristic function of the interval $[0, \Lambda]$. The associated Fourier multiplication operator is denoted by $G_{\Lambda}\left(t, D_{v}\right)$,

$$
\left(G_{\Lambda}\left(t, D_{v}\right) f\right)(t, v):=\int_{\mathbb{R}^{d}} G_{\Lambda}(t, \eta) \hat{f}(t, \eta) e^{2 \pi i \eta \cdot v} \mathrm{~d} \eta=\mathscr{F}^{-1}\left[G_{\Lambda}(t, \cdot) \hat{f}(t, \cdot)\right] .
$$

We use the following convention regarding the Fourier transform of a function $f$ in this article,

$$
(\mathscr{F} f)(\eta)=\hat{f}(\eta)=\int_{\mathbb{R}^{d}} f(v) e^{-2 \pi \mathrm{i} v \cdot \eta} \mathrm{d} v .
$$

The Fourier transform of the Boltzmann operator for Maxwellian molecules has the form (Bobylev identity, [9])

$$
\widehat{Q(g, f)}(\eta)=\int_{\mathbb{S}^{d-1}} b\left(\frac{\eta}{|\eta|} \cdot \sigma\right)\left[\hat{g}\left(\eta^{-}\right) \hat{f}\left(\eta^{+}\right)-\hat{g}(0) \hat{f}(\eta)\right] \mathrm{d} \sigma, \quad \eta^{ \pm}=\frac{\eta \pm|\eta| \sigma}{2},
$$

for $d \geq 2$. There is a similar Bobylev identity for the Kac operator [11],

$$
\widehat{K(g, f})(\eta)=\int_{-\frac{\pi}{4}}^{\frac{\pi}{4}} b_{1}(\theta)\left[\hat{g}\left(\eta^{-}\right) \hat{f}\left(\eta^{+}\right)-\hat{g}(0) \hat{f}(\eta)\right] \mathrm{d} \theta, \quad \eta^{+}=\eta \cos \theta, \eta^{-}=\eta \sin \theta .
$$

A simple, but in a sense important, consequence of Bobylev's identity is that, for all $d \geq 1$,

$$
P_{\Lambda} Q(g, f)=P_{\Lambda} Q\left(P_{\Lambda} g, P_{\Lambda} f\right)
$$

where, for convenience, we put $P_{\Lambda}:=\mathbb{1}_{\Lambda}\left(D_{v}\right)$ for the orthogonal projection onto Fourier 'modes' $|\eta| \leq \Lambda$.

Note also that, since $G_{\Lambda}(t, \cdot)$ has compact support in $\mathbb{R}_{\eta}^{d}$ for any $t>0$, one has

$$
G_{\Lambda} f, G_{\Lambda}^{2} f \in L^{\infty}\left(\left[0, T_{0}\right] ; H^{\infty}\left(\mathbb{R}^{d}\right)\right)
$$

for any finite $T_{0}>0$ and $\Lambda>0$, if $f \in L^{\infty}\left(\left[0, T_{0}\right] ; L^{1}\left(\mathbb{R}^{d}\right)\right)$. This holds, since

$$
\left\|G_{\Lambda} f\right\|_{H^{s}\left(\mathbb{R}_{v}^{d}\right)}^{2} \leq\|\hat{f}\|_{L^{\infty}\left(\mathbb{R}_{\eta}^{d}\right)}^{2}\left\|\langle\cdot\rangle^{s} G_{\Lambda}(t, \cdot)\right\|_{L^{2}\left(\mathbb{R}_{\eta}^{d}\right)}^{2} \leq\|f\|_{L^{1}\left(\mathbb{R}_{v}^{d}\right)}^{2}\left\|\langle\cdot\rangle^{s} G_{\Lambda}\left(T_{0}, \cdot\right)\right\|_{L^{2}\left(\mathbb{R}_{\eta}^{d}\right)}^{2}, \quad \text { for all } s \geq 0 .
$$

These functions, due to the cut-off in Fourier space, are even analytic in a strip containing $\mathbb{R}_{v}^{d}$.

Theorem 1.6 (Gevrey smoothing I). Assume that the cross-section b satisfies the singularity condition (3) and the integrability condition (4) for $d \geq 2$, and for $d=1, b_{1}$ satisfies the singularity condition (6) and the integrability condition (7) for some $0<v<1$. Let $f$ be a weak solution of the Cauchy problem (1) with initial datum satisfying conditions (8). Then, for all $0<\alpha \leq \min \left\{\alpha_{2, d}, \nu\right\}$,

$$
f(t, \cdot) \in G^{\frac{1}{2 \alpha}}\left(\mathbb{R}^{d}\right)
$$

for all $t>0$, where $\alpha_{2, d}=\frac{\log [(8+d) /(4+d)]}{\log 2}$.

Remarks 1.7. (i) In numbers, $\alpha_{2,1} \simeq 0.847997, \alpha_{2,2} \simeq 0.736966$, and $\alpha_{2,3} \simeq 0.652077$. This means, that under only physically reasonable assumptions of finite mass, energy, and entropy, weak solutions are analytic for $v \geq \frac{1}{2}$ and even ultra-analytic if $v>\frac{1}{2}$. It is easy to see that $\alpha_{2, d}$ is decreasing in $d$ and for $d=6, \alpha_{2,6} \simeq 0.485427$, hence, for $d \geq 6$, analyticity (respectively ultra-analyticity) does not follow from this theorem.

(ii) For the proof of Theorem 1.6 (and also 1.8 and 1.9 below) it is important that the energy of $f$ is bounded, which enters in the technical Lemma 2.14 and its Corollary 2.15. A considerably simpler proof could be given using only that $f \in L_{1}^{1}\left(\mathbb{R}^{d}\right)$. In this case, $\alpha_{2, d}$ is replaced by $\alpha_{1, d}=\frac{\log [(4+d) /(2+d)]}{\log 2}$ (see also Remark 1.10 below). However, $\alpha_{1,3}<0.4855$ in three dimensions, thus we would not be able to conclude (ultra-)analytic smoothing of weak solutions for strong singularities $\frac{1}{2} \leq v<1$. 
(iii) As our theorem above shows, weak solutions of the homogenous Kac equation become Gevrey regular for strictly positive times for moderately singular collision kernels with singularity $v \in\left(0, \frac{1}{2}\right)$, see $(6)$ for the precise description of the singularity, for $v=\frac{1}{2}$ they become analytic, which improves the result of Glangetas and NAJEME [19] in this critical case, and even ultra-anaytic for $v \in\left(\frac{1}{2}, 1\right)$.

(iv) Rotationally symmetric solutions $f$ corresponding to rotationally symmetric initial conditions $f_{0}$ are Gevrey regular for strictly positive times under the same conditions as in the one-dimensional case $d=1$. The proof is exactly as the proof of Theorem 3.1 with some small changes in the proof of Lemma 2.27 where the independence of the solution $f$ on the angular coordinates can be explicitly used with the $n=1$ version of Corollary 2.15.

As already remarked, the result of Theorem 1.6 deteriorate in the dimension. Under the same assumptions, but using quite a bit more structure of the Boltzmann operator, we can prove a dimension independent version. Its proof is considerably more involved than the proof of Theorem 1.6 .

Theorem 1.8 (Gevrey smoothing II). Let $d \geq 2$. Assume that the cross-section $b$ satisfies the conditions of Theorem 1.6. Let $f$ be a weak solution of the Cauchy problem (1) with initial datum satisfying conditions (8). Then, for all $0<\alpha \leq \min \left\{\alpha_{2,2}, \nu\right\}$,

$$
f(t, \cdot) \in G^{\frac{1}{2 \alpha}}\left(\mathbb{R}^{d}\right)
$$

for all $t>0$, where $\alpha_{2,2}=\frac{\log (5 / 3)}{\log 2} \simeq 0.736966$. In particular, in contrast to Theorem 1.6, the weak solution is real analytic if $v=\frac{1}{2}$ and ultra-analytic if $v>\frac{1}{2}$ in any dimension.

If the integrability conditions (4) is replaced by the slightly stronger condition that $b(\cos \theta)$ is bounded away from $\theta=0$, that is,

for any $0<\theta_{0}<\frac{\pi}{2}$ there exists $C_{\theta_{0}}<\infty$ such that $0 \leq b(\cos \theta) \leq C_{\theta_{0}}$ for all $\theta_{0} \leq \theta \leq \frac{\pi}{2}$,

which is true in all physically relevant cases, we can prove an even stronger result.

Theorem 1.9 (Gevrey smoothing III). Let $d \geq 2$. Assume that the cross-section $b$ satisfies the conditions of Theorem 1.6 and the condition (16), that is, it is bounded away from the singularity. Let $f$ be a weak solution of the Cauchy problem (1) with initial datum satisfying conditions (8). Then, for all $0<\alpha \leq \min \left\{\alpha_{2,1}, \nu\right\}$,

$$
f(t, \cdot) \in G^{\frac{1}{2 \alpha}}\left(\mathbb{R}^{d}\right)
$$

for all $t>0$, where $\alpha_{2,1}=\frac{\log (9 / 5)}{\log 2} \simeq 0.847997$.

Remark 1.10. (i) Since we do not rely on interpolation inequalities between Sobolev spaces, our results also include the limiting case $\alpha=v$, at least if $v \leq \alpha_{2, n}(n=d, 2,1)$. This is in contrast to all previous results on smoothing properties of the Boltzmann and Kac equations.

(ii) If higher moments of the initial datum are bounded (and thus stay bounded eternally due to moment propagation results, see, for instance, VILLANI's review [40]), the results in Theorem 1.8 and Theorem 1.9 can be improved in the high singularity case, where $v$ is close to one. Namely, let $f_{0} \in L \log L \cap L_{m}^{1}\left(\mathbb{R}^{d}\right)$ for some integer $m>2$, then the constants $\alpha_{2, d}, \alpha_{2,2}$, respectively $\alpha_{2,1}$ are replaced by $\alpha_{m, n}=\frac{\log [(4 m+n) /(2 m+n)]}{\log 2}(n=d, 2,1)$, which are strictly increasing towards the limit $\alpha_{\infty, n}=1$ as $m$ becomes large. See Theorems 3.1, 3.2 and 3.3 below.

Moreover, we prove that for very strong singularities $v$, we can prescribe precise conditions on the initial datum such that we have $f \in G^{\frac{1}{2 v}}\left(\mathbb{R}^{d}\right)$. 
Theorem 1.11. Given $0<v<1$, there is $m(v)$ such that, if $m \in \mathbb{N}$ and $m \geq m(v)$ and $f_{0} \in$ $L \log L \cap L_{m}^{1}$, the weak solution is in $G^{\frac{1}{2 v}}\left(\mathbb{R}^{d}\right)$ for all $t>0$.

More precisely, under the conditions of Theorem 1.6 having $m \geq \max \left(2, \frac{2^{v}-1}{2-2^{v}}\right)$ yields Gevrey smoothing of order $\frac{1}{2 v}$ and under the slightly stronger conditions of Theorem 1.9 having $m \geq$ $\max \left(2, \frac{2^{v}-1}{2\left(2-2^{v}\right)}\right)$ is enough.

Remark 1.12. The proof of this Theorem follows directly from the results of Theorems 3.1, 3.2, and 3.3 in Section 3, which extend Theorems 1.6, 1.8, and 1.9 to the case of finite moments $m \geq 2$.

The strategy of the proofs of our main results Theorems 1.6, 1.8, and 1.9 is as follows: We start with the additional assumption $f_{0} \in L^{2}$ on the initial datum. We use the known $H^{\infty}$ smoothing of the non-cutoff Boltzmann and Kac equation to allow this. This yields an $L^{2}$ reformulation of the weak formulation of the Boltzmann and Kac equations which includes suitable growing Fourier multipliers.

The inclusion of sub-Gaussian Fourier multipliers leads to a nonlocal and nonlinear commutator of the Boltzmann and Kac kernels, which turns out to be a three-linear expression in the weighted solution $\hat{f}$ on the Fourier side. In order to bound this expression with $L^{2}$ norms, one of the three terms has to be controlled pointwise, including a sub-Gaussian growing factor, see Proposition 2.9. The problem is that one has to control the pointwise bound with an $L^{2}$ norm, which is in general impossible. To overcome this obstacle there are several important technical steps:

(1) When working on a ball of radius $\Lambda$, we need this uniform control only on a a ball of radius $\Lambda / \sqrt{2}$, which enables an inductive procedure.

(2) Using the additional a priori information that the kinetic energy is finite, or, depending on the initial condition, even higher moments are finite, we transform weighted $L^{2}$ bounds into pointwise bounds on slightly smaller balls with an additional loss of power in the weights in Fourier space. Here we rely on Kolmogorov-Landau type inequalities, see Lemma 2.18 and appendix $\mathrm{C}$.

(3) Use of strict concavity of the Fourier multipliers, see Lemma 2.6, in order to compensate for this loss of power.

(4) Averaging over a codimension 2 sphere, in the proof of Theorem 1.8, which allows us to get, in any dimension, the same results as for the two dimensional Boltzmann equation.

(5) Averaging over a codimension 1 set constructed from a codimension 2 sphere and the collision angles $\theta$ away from the singularity, and using the fact that near the singularity, one of the three Fourier weights is not big due to Lemma 2.6, enables us to get, in any dimension, the same results as for the one-dimensional Kac equation under the conditions of Theorems 1.9 and 3.3 .

\section{GeVRey REGULARITY AND (ULTRA-)ANALYTICITY OF WEAK SOLUTIONS WITH $L^{2}$ INITIAL DATA}

In this section, we will prove the Gevrey smoothing of weak solutions with initial datum $f_{0}$ satisfying (8) and, additionally, $f_{0} \in L^{2}\left(\mathbb{R}^{d}\right)$.

2.1. $L^{2}$-Reformulation of the homogeneous Boltzmann equation for weak solutions and coercivity. The following is our starting point for the proof of the regularizing properties of the homogenous Boltzmann equation.

Proposition 2.1. Let $f$ be a weak solution of the Cauchy problem (1) with initial datum $f_{0}$ satisfying (8), and let $T_{0}>0$. Then for all $t \in\left(0, T_{0}\right], \beta>0, \alpha \in(0,1)$, and $\Lambda>0$ we have $G_{\Lambda} f \in$ $\mathscr{C}\left(\left[0, T_{0}\right] ; L^{2}\left(\mathbb{R}^{d}\right)\right)$ and

$$
\begin{aligned}
& \frac{1}{2}\left\|G_{\Lambda}\left(t, D_{v}\right) f(t, \cdot)\right\|_{L^{2}}^{2}-\frac{1}{2} \int_{0}^{t}\left\langle f(\tau, \cdot),\left(\partial_{\tau} G_{\Lambda}^{2}\left(\tau, D_{v}\right)\right) f(\tau, \cdot)\right\rangle \mathrm{d} \tau \\
& =\frac{1}{2}\left\|\mathbb{1}_{\Lambda}\left(D_{v}\right) f_{0}\right\|_{L^{2}}^{2}+\int_{0}^{t}\left\langle Q(f, f)(\tau, \cdot), G_{\Lambda}^{2}\left(\tau, D_{v}\right) f(\tau, \cdot)\right\rangle \mathrm{d} \tau .
\end{aligned}
$$


Informally, equation (18) follows from using $\varphi(t, \cdot):=G_{\Lambda}^{2}\left(t, D_{v}\right) f(t, \cdot)$ in the weak formulation of the homogenous Boltzmann equation. Recall that $G_{\Lambda}^{2} f \in L^{\infty}\left(\left[0, T_{0}\right] ; H^{\infty}\left(\mathbb{R}^{d}\right)\right)$ for any finite $T_{0}>0$, so it misses the required regularity in time needed to be used as a test function. The proof of Proposition 2.1 is analogous to Мовімото et al. [30], for the sake of completeness and the convenience of the reader, we prove it in appendix A.

The coercive properties of the non-cutoff Boltzmann bilinear operator which play the crucial role in the smoothing of solutions are made precise in the following sub-elliptic estimate by AlEXANDRE, Desvillettes, Villani and Wennberg [3]. We remark that, while the proof there is given for the Boltzmann equation, it equally applies to the Kac equation.

Lemma 2.2 (Sub-elliptic Estimate, [3]). Let $g \in L_{2}^{1}\left(\mathbb{R}^{d}\right) \cap L \log L\left(\mathbb{R}^{d}\right), g \geq 0(g \not \equiv 0)$. Assume that the collision cross-section $b$ satisfies (3)-(4) or (6)-(7) respectively, with $0<v<1$. Then there exists a constant $C_{g}>0$ (depending only on the dimension $d$, the collision kernel $b,\|g\|_{L_{2}^{1}}$ and $\|g\|_{L} \log L$ ) and a constant $C>0$ (depending only on $d$ and $b$ ), such that for any $f \in H^{1}\left(\mathbb{R}^{d}\right)$ one has

$$
-\langle Q(g, f), f\rangle \geq C_{g}\|f\|_{H^{v}}^{2}-C\|g\|_{L_{2}^{1}}\|f\|_{L^{2}}^{2} \text {. }
$$

Remark 2.3. As explained for instance in [6], the constant $C_{g}$ is an increasing function of $\|g\|_{L^{1}}$, $\|g\|_{L_{2}^{1}}^{-1}$ and $\|g\|_{L \log L}^{-1}$. In particular, if $g$ is a weak solution of the Cauchy problem (1) with initial datum $g_{0} \in L_{2}^{1}\left(\mathbb{R}^{d}\right) \cap L \log L\left(\mathbb{R}^{d}\right)$, we have $\|g\|_{L^{1}}=\left\|g_{0}\right\|_{L^{1}},\|g\|_{L_{2}^{1}} \leq\left\|g_{0}\right\|_{L_{2}^{1}}$ and $\|g\|_{L \log L} \leq \log 2\left\|g_{0}\right\|_{L^{1}}+$ $H\left(g_{0}\right)+C_{\delta, d}\left\|g_{0}\right\|_{L_{2}^{1}}^{1-\delta}$, for small enough $\delta>0$ (see (86)). This implies $C_{g} \geq C_{g_{0}}$ and thus

$$
-\langle Q(g, f), f\rangle \geq C_{g}\|f\|_{H^{v}}^{2}-C\|g\|_{L_{2}^{1}}\|f\|_{L^{2}}^{2} \geq C_{g_{0}}\|f\|_{H^{v}}^{2}-C\left\|g_{0}\right\|_{L_{2}^{1}}\|f\|_{L^{2}}^{2} .
$$

uniformly in $t \geq 0$.

Together with Proposition 2.1 the coercivity estimate Lemma 2.2 implies

Corollary 2.4 (A priori bound for weak solutions). Let $f$ be a weak solution of the Cauchy problem (1) with initial datum $f_{0}$ satisfying (8), and let $T_{0}>0$. Then there exist constants $\widetilde{C}_{f_{0}}, C_{f_{0}}>0$ (depending only on the dimension $d$, the collision kernel $b,\left\|f_{0}\right\|_{L_{2}^{1}}$ and $\left\|f_{0}\right\|_{L \log L}$ ) such that for all $t \in\left(0, T_{0}\right], \beta>0, \alpha \in(0,1)$, and $\Lambda>0$ we have

$$
\begin{aligned}
\left\|G_{\Lambda} f\right\|_{L^{2}}^{2} \leq\left\|\mathbb{1}_{\Lambda}\left(D_{v}\right) f_{0}\right\|_{L^{2}}^{2} & +\int_{0}^{t} 2\left(-\widetilde{C}_{f_{0}}\left\|G_{\Lambda} f\right\|_{H^{v}}^{2}+C_{f_{0}}\left\|G_{\Lambda} f\right\|_{L^{2}}^{2}\right) \mathrm{d} \tau \\
& +\int_{0}^{t} 2\left|\left\langle Q\left(f, G_{\Lambda} f\right)-G_{\Lambda} Q(f, f), G_{\Lambda} f\right\rangle\right| \mathrm{d} \tau \\
& +\int_{0}^{t} 2 \beta\left\|G_{\Lambda} f\right\|_{H^{\alpha}}^{2} \mathrm{~d} \tau .
\end{aligned}
$$

Proof. We want to apply the coercivity result from Lemma 2.2 to the second integral on the right hand side of Proposition 2.1. Therefore, we write

$$
\begin{aligned}
\left\langle Q(f, f), G_{\Lambda}^{2} f\right\rangle & =\left\langle G_{\Lambda} Q(f, f), G_{\Lambda} f\right\rangle=\left\langle Q\left(f, G_{\Lambda} f\right), G_{\Lambda} f\right\rangle+\left\langle G_{\Lambda} Q(f, f)-Q\left(f, G_{\Lambda} f\right), G_{\Lambda} f\right\rangle \\
& \leq-\widetilde{C}_{f_{0}}\left\|G_{\Lambda} f\right\|_{H^{v}}^{2}+\underbrace{C\left\|f_{0}\right\|_{L_{2}^{1}}}_{=: C_{f_{0}}}\left\|G_{\Lambda} f\right\|_{L^{2}}^{2}+\left\langle G_{\Lambda} Q(f, f)-Q\left(f, G_{\Lambda} f\right), G_{\Lambda} f\right\rangle .
\end{aligned}
$$

Moreover,

$$
\partial_{\tau} G_{\Lambda}^{2}(\tau, \eta)=2 \beta\langle\eta\rangle^{2 \alpha} G_{\Lambda}(t, \eta)
$$

Inserting those two results into (18), we obtain

$$
\begin{aligned}
\left\|G_{\Lambda} f\right\|_{L^{2}}^{2} \leq\left\|\mathbb{1}_{\Lambda}\left(D_{v}\right) f_{0}\right\|_{L^{2}}^{2} & +2 \beta \int_{0}^{t}\left\|G_{\Lambda} f(\tau, \cdot)\right\|_{H^{\alpha}}^{2} \mathrm{~d} \tau+2 \int_{0}^{t}\left(-\widetilde{C}_{f_{0}}\left\|G_{\Lambda} f\right\|_{H^{v}}^{2}+C_{f_{0}}\left\|G_{\Lambda} f\right\|_{L^{2}}^{2}\right) \mathrm{d} \tau \\
& +2 \int_{0}^{t}\left\langle G_{\Lambda} Q(f, f)-Q\left(f, G_{\Lambda} f\right), G_{\Lambda} f\right\rangle \mathrm{d} \tau .
\end{aligned}
$$


Remark 2.5. It is natural to call the term $\left\langle G_{\Lambda} Q(f, f)-Q\left(f, G_{\Lambda} f\right), G_{\Lambda} f\right\rangle$ the commutation error.

2.2. Bound on the commutation error. Next, we prove a new bound on the commutation error. An important ingredient is the following elementary observation:

Lemma 2.6 (Strict concavity bound). Let $\alpha \in(0,1]$ be fixed. The map $0 \leq u \mapsto \epsilon(\alpha, u):=$ $(1+u)^{\alpha}-u^{\alpha}$ has the following properties:

(i) If $\alpha \in(0,1)$, then $\epsilon(\alpha, \cdot)$ is strictly decreasing on $[0, \infty)$ with $\lim _{u \rightarrow \infty} \epsilon(\alpha, u)=0$.

In particular, for any $\gamma \geq 1$ and $0 \leq \gamma s^{-} \leq s^{+}$one has

$$
\epsilon\left(\alpha, \frac{s^{+}}{s^{-}}\right) \leq \epsilon(\alpha, \gamma) \leq \epsilon(\alpha, 1)=2^{\alpha}-1<1 .
$$

Moreover, for all $\alpha \in(0,1)$ and all $u>0$

$$
\epsilon(\alpha, u) \leq u^{\alpha-1} .
$$

(ii) If $u>0$, then $\epsilon(\cdot, u)$ is strictly increasing on $[0,1]$.

(iii) For all $s^{-}, s^{+} \geq 0$

$$
\left(1+s^{-}+s^{+}\right)^{\alpha} \leq \epsilon\left(\alpha, \frac{s^{+}}{s^{-}}\right)\left(1+s^{-}\right)^{\alpha}+\left(1+s^{+}\right)^{\alpha} .
$$

Proof. Since

$$
\frac{\partial}{\partial u} \epsilon(\alpha, u)=\alpha\left((1+u)^{\alpha-1}-u^{\alpha-1}\right)<0 \quad \text { for } \alpha \in(0,1)
$$

$\epsilon(\alpha, \cdot)$ is strictly decreasing. Furthermore, for fixed $u>0$ we have

$$
\frac{\partial}{\partial \alpha} \epsilon(\alpha, u)=\log (1+u)(1+u)^{\alpha}-\log u u^{\alpha}>0,
$$

which shows that $\epsilon(\cdot, u)$ is strictly increasing.

For $\alpha \in(0,1)$ and $u \geq 0$ we estimate

$$
\epsilon(u, \alpha)=\alpha \int_{u}^{1+u} r^{\alpha-1} \mathrm{~d} r \leq \alpha u^{\alpha-1} \leq u^{\alpha-1} .
$$

In particular, $\lim _{u \rightarrow \infty} \epsilon(\alpha, u)=0$. By monotonicity, the chain of inequalities (20) follows.

Let $s^{-}, s^{+} \geq 0$. Then

$$
\begin{aligned}
\left(1+s^{-}+s^{+}\right)^{\alpha} & =\left(s^{-}\right)^{\alpha}\left[\left(1+\frac{1+s^{+}}{s^{-}}\right)^{\alpha}-\left(\frac{1+s^{+}}{s^{-}}\right)^{\alpha}\right]+\left(1+s^{+}\right)^{\alpha} \\
& \leq \epsilon\left(\alpha, \frac{1+s^{+}}{s^{-}}\right)\left(1+s^{-}\right)^{\alpha}+\left(1+s^{+}\right)^{\alpha} \leq \epsilon\left(\alpha, \frac{s^{+}}{s^{-}}\right)\left(1+s^{-}\right)^{\alpha}+\left(1+s^{+}\right)^{\alpha}
\end{aligned}
$$

where we made use of the monotonicity of $\epsilon(\alpha, \cdot)$ in the last inequality.

Remark 2.7. The proof of Lemma 2.6 is so simple that one might wonder whether it could be of any use. In fact, it is crucial. It's usefulness is hidden in the fact that it enables us to gain a small exponent in the commutator estimates, see Proposition 2.9 and Lemma 2.11 below. Furthermore, $\epsilon(\alpha, \gamma)$ can be made as small as we like if $\gamma$ can be chosen large enough, which will be important in the proof of Theorem 1.9 .

Corollary 2.8. Let $\widetilde{G}(s):=e^{\beta t(1+s)^{\alpha}}$ for $s \geq 0, \alpha \in(0,1]$. Then, for all $s^{-}+s^{+}=s$ with $0 \leq s^{-} \leq s^{+}$,

$$
\left|\widetilde{G}(s)-\widetilde{G}\left(s^{+}\right)\right| \leq 2 \alpha \beta t\left(1+s^{+}\right)^{\alpha}\left(1-\frac{s^{+}}{s}\right) \widetilde{G}\left(s^{-}\right)^{\epsilon\left(\alpha, \frac{s^{+}}{s^{-}}\right)} \widetilde{G}\left(s^{+}\right)
$$

with $\epsilon(\alpha, u)$ from Lemma 2.6.

Proof. Since $s^{+} \leq s$ and $\alpha \in(0,1]$,

$$
\left|\widetilde{G}(s)-\widetilde{G}\left(s^{+}\right)\right| \leq \int_{s^{+}}^{s}\left|\frac{\mathrm{d}}{\mathrm{d} r} \widetilde{G}(r)\right| \mathrm{d} r=\alpha \beta t \int_{s^{+}}^{s}(1+r)^{\alpha-1} \widetilde{G}(r) \mathrm{d} r \leq \alpha \beta t\left(1+s^{+}\right)^{\alpha-1}\left(s-s^{+}\right) \widetilde{G}(s) .
$$

In addition, since $s \leq 2 s^{+}$,

$$
\frac{s-s^{+}}{1+s^{+}}=\left(1-\frac{s^{+}}{s}\right) \frac{s}{1+s^{+}} \leq 2\left(1-\frac{s^{+}}{s}\right) .
$$


Moreover, since $s=s^{+}+s^{-}$, the strict concavity Lemma 2.6 gives

$$
\widetilde{G}(s) \leq \widetilde{G}\left(s^{-}\right)^{\epsilon\left(\alpha, \frac{s^{+}}{s^{-}}\right)} \widetilde{G}\left(s^{+}\right),
$$

which completes the proof.

Proposition 2.9 (Bound on Commutation Error). Let $f$ be a weak solution of the Cauchy problem (1) with initial datum $f_{0}$ satisfying (8). Recall $\epsilon(\alpha, u)=(1+u)^{\alpha}-u^{\alpha}$. Then for all $t \in\left(0, T_{0}\right], \beta>0$, $\alpha \in(0,1)$, and $\Lambda>0$ we have

$$
\begin{aligned}
&\left|\left\langle Q\left(f, G_{\Lambda} f\right)-G_{\Lambda} Q(f, f), G_{\Lambda} f\right\rangle\right| \\
& \leq 2 \alpha \beta t \int_{\mathbb{R}^{d}} \int_{\mathbb{S}^{d-1}} b\left(\frac{\eta}{|\eta|} \cdot \sigma\right)\left(1-\frac{\left|\eta^{+}\right|^{2}}{|\eta|^{2}}\right) G\left(\eta^{-}\right)^{\epsilon\left(\alpha,\left|\eta^{+}\right|^{2} /\left|\eta^{-}\right|^{2}\right)}\left|\hat{f}\left(\eta^{-}\right)\right| \\
& \times G_{\Lambda}\left(\eta^{+}\right)\left|\hat{f}\left(\eta^{+}\right)\right| G_{\Lambda}(\eta)|\hat{f}(\eta)|\left\langle\eta^{+}\right\rangle^{2 \alpha} \mathrm{d} \sigma \mathrm{d} \eta,
\end{aligned}
$$

for $d \geq 2$, and

$$
\begin{aligned}
&\left|\left\langle Q\left(f, G_{\Lambda} f\right)-G_{\Lambda} Q(f, f), G_{\Lambda} f\right\rangle\right| \\
& \leq 2 \alpha \beta t \int_{\mathbb{R}} \int_{-\frac{\pi}{4}}^{\frac{\pi}{4}} b_{1}(\theta) \sin ^{2} \theta G\left(\eta^{-}\right)^{\epsilon\left(\alpha,\left.\left|\eta^{+}\right|\right|^{2} /\left|\eta^{-}\right|^{2}\right)}\left|\hat{f}\left(\eta^{-}\right)\right| \\
& \times G_{\Lambda}\left(\eta^{+}\right)\left|\hat{f}\left(\eta^{+}\right)\right| G_{\Lambda}(\eta)|\hat{f}(\eta)|\left\langle\eta^{+}\right\rangle^{2 \alpha} \mathrm{d} \theta \mathrm{d} \eta,
\end{aligned}
$$

in the one-dimensional case.

Remark 2.10. If the weight $G$ was growing polynomially, the term $G\left(\eta^{-}\right)$in the integral (21), respectively (22), would be replaced by 1 . In this case, the "bad terms" which contain $\eta^{-}$can simply be bounded by $\|\hat{f}\|_{L^{\infty}} \leq\|f\|_{L^{1}}=\left\|f_{0}\right\|_{L^{1}}$ and the rest can be bounded nicely in terms of $\left\|G_{\Lambda} \hat{f}\right\|_{L^{2}}$ and $\left\|G_{\Lambda} \hat{f}\right\|_{H^{\alpha}}$, see the discussion in appendix B.

If the weight $G$ is exponential, the estimate of the terms containing $\eta^{-}$in (21), respectively (22), is an additional challenge and the methods we devised in order to control this term in the commutation error is probably the most important new contribution of this work.

Proof of Proposition 2.9. We start with $d \geq 2$. By Bobylev's identity, one has

$$
\begin{aligned}
& \left|\left\langle Q\left(f, G_{\Lambda} f\right)-G_{\Lambda} Q(f, f), G_{\Lambda} f\right\rangle\right|=\left|\left\langle\mathscr{F}\left[Q\left(f, G_{\Lambda} f\right)-G_{\Lambda} Q(f, f)\right], \mathscr{F}\left[G_{\Lambda} f\right]\right\rangle_{L^{2}}\right| \\
& \quad \leq \int_{\mathbb{R}^{d}} \int_{\mathbb{S}^{d-1}} b\left(\frac{\eta}{|\eta|} \cdot \sigma\right) G_{\Lambda}(\eta)|\hat{f}(\eta)|\left|\hat{f}\left(\eta^{-}\right)\right|\left|\hat{f}\left(\eta^{+}\right)\right|\left|G_{\Lambda}\left(\eta^{+}\right)-G_{\Lambda}(\eta)\right| \mathrm{d} \sigma \mathrm{d} \eta \\
& \quad=\int_{\mathbb{R}^{d}} \int_{\mathbb{S}^{d-1}} b\left(\frac{\eta}{|\eta|} \cdot \sigma\right) G_{\Lambda}(\eta)|\hat{f}(\eta)|\left|\hat{f}\left(\eta^{-}\right)\right|\left|\hat{f}\left(\eta^{+}\right)\right|\left|G\left(\eta^{+}\right)-G(\eta)\right| \mathrm{d} \sigma \mathrm{d} \eta,
\end{aligned}
$$

where the latter equality follows from the fact that $G_{\Lambda}$ is supported on the ball $\{|\eta| \leq \Lambda\}$ and $\left|\eta^{+}\right| \leq|\eta|$.

To estimate $\left|G\left(\eta^{+}\right)-G(\eta)\right|$, we use Corollary 2.8 with $s:=|\eta|^{2}$ and, accordingly, $s^{ \pm}=\left|\eta^{ \pm}\right|^{2}$. Notice that

$$
\left|\eta^{ \pm}\right|^{2}=\frac{|\eta|^{2}}{2}\left(1 \pm \frac{\eta}{|\eta|} \cdot \sigma\right), \quad|\eta|^{2}=\left|\eta^{+}\right|^{2}+\left|\eta^{-}\right|^{2}
$$

and, writing $\cos \theta=\frac{\eta \cdot \sigma}{|\eta|}$, we also have

$$
\left|\eta^{+}\right|^{2}=|\eta|^{2} \cos ^{2} \frac{\theta}{2}, \quad\left|\eta^{-}\right|^{2}=|\eta|^{2} \sin ^{2} \frac{\theta}{2}
$$

Since $b$ is supported on angles in $[0, \pi / 2]$, one sees $0 \leq\left|\eta^{-}\right|^{2} \leq \frac{1}{2}|\eta|^{2}$ and $\frac{1}{2}|\eta|^{2} \leq\left|\eta^{+}\right|^{2} \leq|\eta|^{2}$. Therefore, $s^{-} \leq \frac{s}{2} \leq s^{+} \leq s$ and $s=s^{+}+s^{-}$.

It follows that for all $\eta \in \mathbb{R}^{d}$ with $|\eta| \leq \Lambda$, noting that $\left|\eta^{+}\right| \leq|\eta| \leq \Lambda$,

$$
\left|G(\eta)-G\left(\eta^{+}\right)\right| \leq 2 \alpha \beta t\left\langle\eta^{+}\right\rangle^{2 \alpha}\left(1-\frac{\left|\eta^{+}\right|^{2}}{|\eta|^{2}}\right) G\left(\eta^{-}\right)^{\epsilon\left(\alpha,\left|\eta^{+}\right|^{2} /\left|\eta^{-}\right|^{2}\right)} G_{\Lambda}\left(\eta^{+}\right),
$$

which finishes the proof in dimension $d \geq 2$. 
For the Kac model we remark that the above proof depends only on $\left|\eta^{-}\right| \leq\left|\eta^{+}\right| \leq|\eta|$ and $\left|\eta^{-}\right|^{2}+$ $\left|\eta^{+}\right|^{2}=|\eta|^{2}$, hence $\left|\eta^{-}\right|^{2} \leq|\eta|^{2} / 2$, and the strict concavity Lemma 2.6 and the Corollary 2.8. Since, by symmetry, we assume that $b_{1}$ is supported in $[-\pi / 4, \pi / 4]$, the same bounds for $\eta^{-}$and $\eta^{+}$hold in dimension one and the above proof can be literally translated, with obvious changes in notation, to the Kac equation.

The bound on the commutation error in Proposition 2.9 is a trilinear expression in the weak solution $f$. In order to close the a priori bound from Corollary 2.4 in $L^{2}$, one of the terms has to be controlled uniformly in $\eta$. Seemingly impossible with the growing weights, it is exactly at this place where the gain of the small exponent $\epsilon\left(\alpha,\left|\eta^{+}\right|^{2} /\left|\eta^{-}\right|^{2}\right) \leq \epsilon(\alpha, 1)<1$ in the $G\left(\eta^{-}\right)$term in (21) and (22) allows us to proceed with this strategy. This gain of the small exponent is new and enabled by the strict concavity bound of Lemma 2.6 and its Corollary 2.8 and it is crucial for our inductive approach for controlling the commutation error.

Lemma 2.11. The inequality

$$
\left|\left\langle Q\left(f, G_{\Lambda} f\right)-G_{\Lambda} Q(f, f), G_{\Lambda} f\right\rangle\right| \leq I_{d, \Lambda}+I_{d, \Lambda}^{+}
$$

holds, where, for $d \geq 2$

$$
\begin{gathered}
I_{d, \Lambda}=\alpha \beta t \int_{\mathbb{R}^{d}} \int_{0}^{\frac{\pi}{2}} \int_{\mathbb{S}^{d-2}(\eta)} \sin ^{d} \theta \\
\times(\cos \theta) G\left(\eta^{-}\right)^{\epsilon\left(\alpha, \cot ^{2} \frac{\theta}{2}\right)}\left|\hat{f}\left(\eta^{-}\right)\right| \mathbb{1}_{\frac{\Lambda}{\sqrt{2}}}\left(\left|\eta^{-}\right|\right) \mathrm{d} \omega \mathrm{d} \theta \\
\times\left|G_{\Lambda}(\eta) \hat{f}(\eta)\right|^{2}\langle\eta\rangle^{2 \alpha} \mathrm{d} \eta .
\end{gathered}
$$

Here the vector $\eta^{-}$is expressed as a function of $\eta$ and $\sigma$, that is,

$$
\eta^{-}=\eta^{-}(\eta, \sigma)=\frac{1}{2}(\eta-|\eta| \sigma)=|\eta| \sin ^{2}\left(\frac{\theta}{2}\right) \frac{\eta}{|\eta|}-|\eta| \sin \left(\frac{\theta}{2}\right) \cos \left(\frac{\theta}{2}\right) \omega
$$

and $\sigma$ is is a vector on the unit sphere given by

$$
\sigma=\sigma(\theta, \omega)=\cos (\theta) \frac{\eta}{|\eta|}+\sin (\theta) \omega
$$

with polar angle $\theta \in[0, \pi / 2]$ with respect to the north pole in the $\eta$ direction, $\omega \in \mathbb{S}^{d-2}(\eta):=\{\widetilde{\omega} \in$ $\left.\mathbb{R}^{d}: \widetilde{\omega} \perp \eta,|\widetilde{\omega}|=1\right\}$, the $d-2$ sphere in $\mathbb{R}^{d}$ orthogonal to the $\eta$ direction, and $\mathrm{d} \omega$ the canonical measure on $\mathbb{S}^{d-2}$.

$$
\begin{gathered}
I_{d, \Lambda}^{+}=2^{d} \alpha \beta t \int_{\mathbb{R}^{d}} \int_{0}^{\frac{\pi}{4}} \int_{\mathbb{S}^{d-2}\left(\eta^{+}\right)} \sin ^{d} \vartheta b(\cos 2 \vartheta) G\left(\eta^{-}\right)^{\epsilon\left(\alpha, \cot ^{2} \vartheta\right)}\left|\hat{f}\left(\eta^{-}\right)\right| \mathbb{1}_{\frac{\Lambda}{\sqrt{2}}}\left(\left|\eta^{-}\right|\right) \\
\times\left|G_{\Lambda}\left(\eta^{+}\right) \hat{f}\left(\eta^{+}\right)\right|^{2}\left\langle\eta^{+}\right\rangle^{2 \alpha} \mathrm{d} \vartheta \mathrm{d} \omega \mathrm{d} \eta^{+}
\end{gathered}
$$

where now the vector $\eta^{-}$is expressed as a function of $\eta^{+}$and $\sigma$, that is,

$$
\eta^{-}=\eta^{-}\left(\eta^{+}, \sigma\right)=\eta^{+}-\left|\eta^{+}\right|\left(\frac{\eta^{+} \cdot \sigma}{\left|\eta^{+}\right|}\right)^{-1} \sigma=-\left|\eta^{+}\right| \tan (\vartheta) \omega
$$

where now $\sigma$ is is a vector on the unit sphere with north pole in the $\eta^{+}$direction given by

$$
\sigma=\sigma(\vartheta, \omega)=\cos (\vartheta) \frac{\eta^{+}}{\left|\eta^{+}\right|}+\sin (\vartheta) \omega
$$

with polar angle $\vartheta \in[0, \pi / 4]$ and $\omega \in \mathbb{S}^{d-2}\left(\eta^{+}\right)$, the $(d-2)$-sphere in $\mathbb{R}^{d}$ orthogonal to the $\eta^{+}$ direction. If $d=2$ we set $\mathbb{S}^{0}:=\emptyset$ in this context. 
For $d=1$ we have

$$
\begin{gathered}
I_{1, \Lambda}=\alpha \beta t \int_{\mathbb{R}} \int_{-\frac{\pi}{4}}^{\frac{\pi}{4}} \sin ^{2} \theta b_{1}(\theta) G\left(\eta^{-}\right)^{\epsilon\left(\alpha, \cot ^{2} \frac{\theta}{2}\right)}\left|\hat{f}\left(\eta^{-}\right)\right| \mathbb{1}_{\frac{\Lambda}{\sqrt{2}}}\left(\left|\eta^{-}\right|\right) \mathrm{d} \theta \\
\times\left|G_{\Lambda}(\eta) \hat{f}(\eta)\right|^{2}\langle\eta\rangle^{2 \alpha} \mathrm{d} \eta, \\
I_{1, \Lambda}^{+}=\sqrt{2} \alpha \beta t \int_{\mathbb{R}} \int_{-\frac{\pi}{4}}^{\frac{\pi}{4}} \sin ^{2} \theta b_{1}(\theta) \\
G\left(\eta^{-}\right)^{\epsilon\left(\alpha, \cot ^{2} \frac{\theta}{2}\right)}\left|\hat{f}\left(\eta^{-}\right)\right| \mathbb{1}_{\frac{\Lambda}{\sqrt{2}}}\left(\left|\eta^{-}\right|\right) \mathrm{d} \omega \mathrm{d} \theta \\
\times\left|G_{\Lambda}\left(\eta^{+}\right) \hat{f}\left(\eta^{+}\right)\right|^{2}\left\langle\eta^{+}\right\rangle^{2 \alpha} \mathrm{d} \eta^{+},
\end{gathered}
$$

where in the first case $\eta^{-}=\eta^{-}(\eta, \theta)=\eta \sin \theta$ and in the second case $\eta^{-}=\eta^{-}\left(\eta^{+}, \theta\right)=\eta^{+} \tan \theta$ and there is no need to distinguish between the $\theta$ and $\vartheta$ parametrization.

Remark 2.12. In the $\eta$, respectively $\eta^{+}$, integrals above $\eta^{-}$and $\sigma$ are always the same vectors expressed in different parametrizations. We therefore have the relation $\vartheta=\theta / 2$, see Figure 1 for the geometry of the collision process in Fourier space.

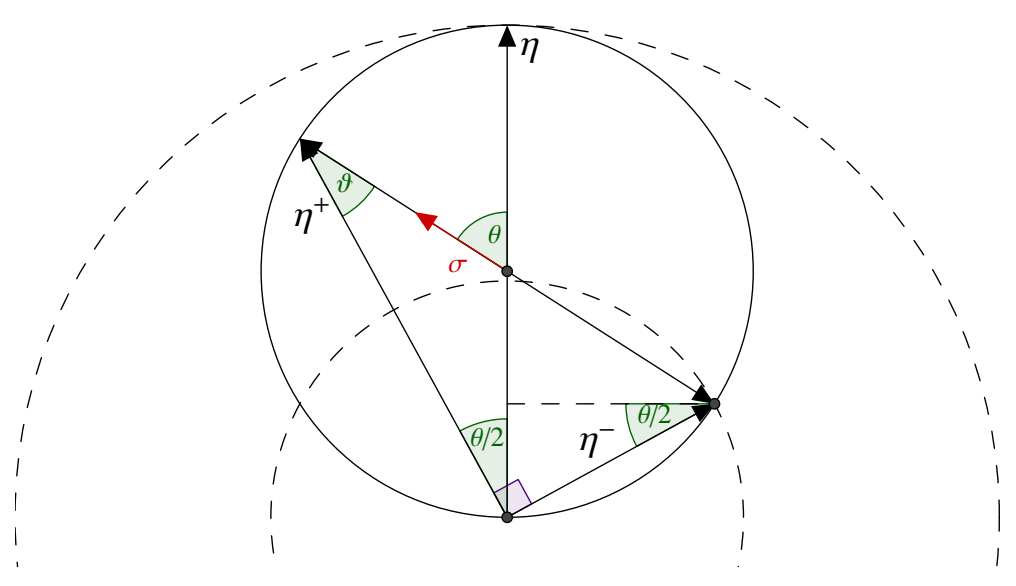

FIGURE 1. Geometry of the collision process in Fourier space.

Remark 2.13. From the bounds given in Lemma 2.11 one might already see that, in order to bound the commutation error by some multiple of $\left\|G_{\Lambda} f\right\|_{H^{\alpha}\left(\mathbb{R}^{d}\right)}^{2}$, one has to control integrals of the form

$$
\sup _{|\eta| \leq \Lambda} \int_{0}^{\frac{\pi}{2}} \int_{\mathbb{S}^{d-2}(\eta)} \sin ^{d} \theta b(\cos \theta) G^{\epsilon\left(\alpha, \cot ^{2} \frac{\theta}{2}\right)}\left(\eta^{-}\right)\left|\hat{f}\left(\eta^{-}\right)\right| \mathbb{1}_{\frac{\Lambda}{\sqrt{2}}}\left(\left|\eta^{-}\right|\right) \mathrm{d} \omega \mathrm{d} \theta,
$$

with the parametrisation (25) for $\eta^{-}$, and similarly for (27) and the corresponding integrals in the one dimensional case. Due to characteristic function in $\eta^{-}$, this uniform control is not needed on the full ball of radius $\Lambda$, but only on a strictly smaller one, giving rise to an induction-over-length-scales type of argument.

Proof of Lemma 2.11. Let $d \geq 2$. Using the elementary estimate

$$
\left|G_{\Lambda}(\eta) \hat{f}(\eta)\right|\left|G_{\Lambda}\left(\eta^{+}\right) \hat{f}\left(\eta^{+}\right)\right| \leq \frac{1}{2}\left(\left|G_{\Lambda}(\eta) \hat{f}(\eta)\right|^{2}+\left|G_{\Lambda}\left(\eta^{+}\right) \hat{f}\left(\eta^{+}\right)\right|^{2}\right)
$$

in the bound (21) gives

$$
\left|\left\langle Q\left(f, G_{\Lambda} f\right)-G_{\Lambda} Q(f, f), G_{\Lambda} f\right\rangle\right| \leq \widetilde{I}_{d, \Lambda}+\widetilde{I}_{d, \Lambda}^{+}
$$


with

$$
\begin{aligned}
& \widetilde{I}_{d, \Lambda}=\alpha \beta t \int_{\mathbb{R}^{d}} \int_{\mathbb{S}^{d-1}} b\left(\frac{\eta}{|\eta|} \cdot \sigma\right)\left(1-\frac{\left|\eta^{+}\right|^{2}}{|\eta|^{2}}\right) G\left(\eta^{-}\right)^{\epsilon\left(\alpha,\left|\eta^{+}\right|^{2} /\left|\eta^{-}\right|^{2}\right)}\left|\hat{f}\left(\eta^{-}\right)\right| \mathbb{1}_{\frac{\Lambda}{\sqrt{2}}}\left(\left|\eta^{-}\right|\right) \\
& \times\left|G_{\Lambda}(\eta) \hat{f}(\eta)\right|^{2}\left\langle\eta^{+}\right\rangle^{2 \alpha} \mathrm{d} \sigma \mathrm{d} \eta,
\end{aligned}
$$

and

$$
\begin{array}{r}
\widetilde{I}_{d, \Lambda}^{+}=\alpha \beta t \int_{\mathbb{R}^{d}} \int_{\mathbb{S}^{d-1}} b\left(\frac{\eta}{|\eta|} \cdot \sigma\right)\left(1-\frac{\left|\eta^{+}\right|^{2}}{|\eta|^{2}}\right) \\
G\left(\eta^{-}\right)^{\epsilon\left(\alpha,\left|\eta^{+}\right|^{2} /\left|\eta^{-}\right|^{2}\right)}\left|\hat{f}\left(\eta^{-}\right)\right| \mathbb{1}_{\frac{\Lambda}{\sqrt{2}}}\left(\left|\eta^{-}\right|\right) \\
\times\left|G_{\Lambda}\left(\eta^{+}\right) \hat{f}\left(\eta^{+}\right)\right|^{2}\left\langle\eta^{+}\right\rangle^{2 \alpha} \mathrm{d} \sigma \mathrm{d} \eta
\end{array}
$$

First we consider $\widetilde{I}_{d, \Lambda}$ : Writing $\sigma$ in a parametrization where the north pole is in the $\eta$ direction, one has

$$
\sigma=\cos \theta \frac{\eta}{|\eta|}+\sin \theta \omega
$$

where $\cos \theta=\frac{\eta \cdot \sigma}{|\eta|} \geq 0$ and $\omega$ is a unit vector orthogonal to $\eta$, that is, $\omega \in \mathbb{S}^{d-2}(\eta)$. Due to the support condition on $b$ one has $\cos \theta \geq 0$, that is, $\sigma$ is restricted to the northern hemisphere $\theta \in[0, \pi / 2]$. In this parametization one has $\mathrm{d} \sigma=\sin ^{d-2} \theta \mathrm{d} \theta \mathrm{d} \omega$. From the definition of $\eta^{ \pm}$one sees

$$
\eta^{ \pm}=\frac{1}{2}(\eta \pm|\eta| \sigma)=\frac{|\eta|}{2}(1 \pm \cos \theta) \frac{\eta}{|\eta|} \pm \frac{|\eta|}{2} \sin (\theta) \omega
$$

so

$$
\eta^{+}=|\eta| \cos ^{2}\left(\frac{\theta}{2}\right) \frac{\eta}{|\eta|}+|\eta| \sin \left(\frac{\theta}{2}\right) \cos \left(\frac{\theta}{2}\right) \omega
$$

In particular,

$$
\left|\eta^{+}\right|=|\eta| \cos \frac{\theta}{2}, \quad \text { and } \quad 1-\frac{\left|\eta^{+}\right|^{2}}{|\eta|^{2}}=1-\cos ^{2} \frac{\theta}{2}=\sin ^{2} \frac{\theta}{2}
$$

Moreover,

$$
\eta^{-}=|\eta| \sin ^{2} \frac{\theta}{2} \frac{\eta}{|\eta|}-|\eta| \sin \frac{\theta}{2} \cos \frac{\theta}{2} \omega, \quad \text { and } \quad\left|\eta^{-}\right|=|\eta| \sin \frac{\theta}{2}
$$

so

$$
\frac{\left|\eta^{+}\right|^{2}}{\left|\eta^{-}\right|^{2}}=\frac{\cos ^{2} \frac{\theta}{2}}{\sin ^{2} \frac{\theta}{2}}=\cot ^{2} \frac{\theta}{2} .
$$

After this preparation, using also $\left\langle\eta^{+}\right\rangle^{2 \alpha} \leq\langle\eta\rangle^{2 \alpha}$ and $\sin \frac{\theta}{2} \leq \sin \theta$ for $\theta \in\left[0, \frac{\pi}{2}\right]$, the inequality $\widetilde{I}_{d, \Lambda} \leq I_{d, \Lambda}$ is immediate. The inclusion of the additional factor $\mathbb{1}_{\Lambda}(|\eta|)=\mathbb{1}_{\sin \frac{\theta}{2} \Lambda}\left(\left|\eta^{-}\right|\right) \leq \mathbb{1}_{\Lambda / \sqrt{2}}\left(\left|\eta^{-}\right|\right)$ seems artificial for the moment, but will be convenient to keep track of the fact that $\eta^{-}$is always restricted to a ball of radius $\frac{\Lambda}{\sqrt{2}}$.

Concerning $\widetilde{I}_{d, \Lambda}$, we want to implement a change of variables from $\eta$ to $\eta^{+}$. As a function of $\eta$ and $\sigma, \eta^{+}=\frac{1}{2}(\eta-|\eta| \sigma)$. Thus

$$
\left|\frac{\partial \eta^{+}}{\partial \eta}\right|=\left|\frac{1}{2}\left(\mathbb{1}+\frac{\eta}{|\eta|} \otimes \sigma\right)\right|=\frac{1}{2^{d}}\left(1+\frac{\eta}{|\eta|} \cdot \sigma\right) \geq \frac{1}{2^{d}},
$$

since $\eta \cdot \sigma \geq 0$ and the second equality is an application of Sylvester's determinant theorem. Therefore, the Jacobian of the transformation from $\eta$ to $\eta^{+}$can be bounded by

$$
\left|\frac{\partial \eta}{\partial \eta^{+}}\right|=\left|\frac{\partial \eta^{+}}{\partial \eta}\right|^{-1} \leq 2^{d}
$$

In addition,

$$
\left|\eta^{+}\right|^{2}=\frac{|\eta|^{2}}{2}\left(1+\frac{\eta \cdot \sigma}{|\eta|}\right) \quad \text { and } \quad \eta^{+} \cdot \sigma=\frac{|\eta|}{2}\left(1+\frac{\eta \cdot \sigma}{|\eta|}\right)=\frac{\left|\eta^{+}\right|^{2}}{|\eta|}
$$


which implies

$$
\frac{\eta^{+} \cdot \sigma}{\left|\eta^{+}\right|}=\frac{\left|\eta^{+}\right|}{|\eta|} \quad \text { and } \quad \frac{\eta \cdot \sigma}{|\eta|}=2 \frac{\left|\eta^{+}\right|^{2}}{|\eta|^{2}}-1=2\left(\frac{\eta^{+} \cdot \sigma}{\left|\eta^{+}\right|}\right)^{2}-1
$$

Moreover, from the definition of $\eta^{ \pm}$one sees

$$
\eta=2 \eta^{+}-|\eta| \sigma
$$

so

$$
\eta^{-}=\eta^{+}-|\eta| \sigma=\eta^{+}-\left|\eta^{+}\right|\left(\frac{\eta^{+} \cdot \sigma}{\left|\eta^{+}\right|}\right)^{-1} \sigma
$$

Therefore, taking care of the domain of integration,

$$
\begin{aligned}
\widetilde{I}_{d}^{+} \leq 2^{d} \int_{\mathbb{R}^{d}} \int_{\mathbb{S}^{d-1}} b\left(2\left(\frac{\eta^{+} \cdot \sigma}{\left|\eta^{+}\right|}\right)^{2}-1\right)\left(1-\left(\frac{\eta^{+} \cdot \sigma}{\left|\eta^{+}\right|}\right)^{2}\right) \mathbb{1}_{\frac{\eta^{+} \cdot \sigma}{\left|\eta^{+}\right|} \Lambda}\left(\left|\eta^{+}\right|\right) \\
\times G^{\epsilon\left(\alpha,\left.\left|\eta^{+}\right|\right|^{2} /\left|\eta^{-}\right|^{2}\right)}\left(\eta^{-}\right)\left|\hat{f}\left(\eta^{-}\right)\right|\left|G_{\Lambda}\left(\eta^{+}\right) \hat{f}\left(\eta^{+}\right)\right|^{2}\left\langle\eta^{+}\right\rangle^{2 \alpha} \mathrm{d} \sigma \mathrm{d} \eta^{+} .
\end{aligned}
$$

Introducing spherical coordinates with north pole in the $\eta^{+}$direction, one has

$$
\sigma=\sigma(\vartheta, \omega)=\cos (\vartheta) \frac{\eta^{+}}{\left|\eta^{+}\right|}+\sin (\vartheta) \omega
$$

where now $\cos \vartheta=\frac{\eta^{+} \cdot \sigma}{\left|\eta^{+}\right|}$. From figure 1 one sees $\vartheta=\frac{\theta}{2} \in[0, \pi / 4]$. In this parametrization one has

$$
\eta^{-}=\eta^{+}-\frac{\left|\eta^{+}\right|}{\cos \vartheta} \sigma=-\left|\eta^{+}\right| \tan (\vartheta) \omega
$$

and again $\mathrm{d} \sigma=\sin ^{d-2} \vartheta \mathrm{d} \vartheta \mathrm{d} \omega$. Thus

$$
\begin{gathered}
\widetilde{I}_{d}^{+} \leq 2^{d} \int_{\mathbb{R}^{d}} \int_{\mathbb{S}^{d-2}} \int_{0}^{\frac{\pi}{4}} b(\cos 2 \vartheta) \sin ^{d} \vartheta G^{\epsilon\left(\alpha, \cot ^{2} \vartheta\right)}\left(\eta^{-}\right)\left|\hat{f}\left(\eta^{-}\right)\right| \mathbb{1}_{(\cos \vartheta) \Lambda}\left(\left|\eta^{+}\right|\right) \\
\times\left|G\left(\eta^{+}\right) \hat{f}\left(\eta^{+}\right)\right|^{2}\left\langle\eta^{+}\right\rangle^{2 \alpha} \mathrm{d} \vartheta \mathrm{d} \omega \mathrm{d} \eta^{+} .
\end{gathered}
$$

Since $\left|\eta^{-}\right|=\left|\eta^{+}\right| \tan \vartheta$ we obtain $\mathbb{1}_{(\cos \vartheta) \Lambda}\left(\left|\eta^{+}\right|\right)=\mathbb{1}_{(\sin \vartheta) \Lambda}\left(\left|\eta^{-}\right|\right) \leq \mathbb{1}_{\Lambda / \sqrt{2}}\left(\left|\eta^{-}\right|\right)$since $\vartheta \in[0, \pi / 4]$. Hence $\widetilde{I}_{d, \Lambda}^{+} \leq I_{d, \Lambda}^{+}$.

The proof in the $d=1$ case is completely analogous.

\subsection{Extracting pointwise information from local $L^{2}$ bounds.}

Lemma 2.14. Let $m \geq 2$ and $h \in W^{m, \infty}(\mathbb{R})$ and $q \geq \frac{1}{m}$. Then there exists a constant $L_{m}<\infty$ depending only on $q, m,\|h\|_{L^{\infty}(\mathbb{R})}$ and $\left\|h^{(m)}\right\|_{L^{\infty}(\mathbb{R})}$ such that

$$
|h(r)|^{q} \leq L_{m} \int_{\Omega_{r}}|h(\xi)|^{q-\frac{1}{m}} \mathrm{~d} \xi \quad \text { for all } r \in \mathbb{R},
$$

where $\Omega_{r}=[r, r+2]$ if $r \geq 0$ and $\Omega_{r}=[r-2, r]$ if $r<0$.

Looking into the proof of Lemma 2.14, it is clear that its $m=1$ version also holds, even with a much simpler proof. Before actually going into the proof, we state an important consequence of it, which will enable us to get pointwise decay estimates on a function once suitable $L^{2}$ norms are bounded.

For $m \in \mathbb{N}$ define $\left\|D^{m} f\right\|_{L^{\infty}\left(\mathbb{R}^{d}\right)}:=\sup _{\omega \in \mathbb{S}^{d-1}}\left\|(\omega \cdot \nabla)^{m} f\right\|_{L^{\infty}\left(\mathbb{R}^{d}\right)}$. Notice that this norm is invariant under rotations of the function $f$.

Corollary 2.15. Let $H \in \mathscr{C}^{m}\left(\mathbb{R}^{n}\right)$. Then there exists a constant $L_{m, n}<\infty$ (depending only on $m, n,\|H\|_{L^{\infty}\left(\mathbb{R}^{n}\right)}$ and, $\left.\left\|D^{m} H\right\|_{L^{\infty}\left(\mathbb{R}^{n}\right)}\right)$ such that

$$
|H(x)| \leq L_{m, n}\left(\int_{Q_{x}}|H(\xi)|^{2} \mathrm{~d} \xi\right)^{\frac{m}{2 m+n}},
$$


where $Q_{x}$ is a cube in $\mathbb{R}^{n}$ of side length 2 , with $x$ being one of the corners, such that it is oriented away from $x$ in the sense that $x \cdot(\xi-x) \geq 0$ for all $\xi \in Q_{x}$.

Remark 2.16. The constant $L_{m, n}$ in Corollary 2.15 is invariant under rotations of the function $H$. This will be convenient for its application in Sections 2.5 and 2.6.

Proof. We apply Lemma 2.14 iteratively in each coordinate direction to obtain

$$
\begin{aligned}
\left|H\left(x_{1}, x_{2}, \ldots, x_{n}\right)\right|^{2+\frac{n}{m}} & \leq L_{m}^{(1)} \int_{\Omega_{x_{1}}}\left|H\left(\xi_{1}, x_{2}, \ldots, x_{d}\right)\right|^{2+\frac{n-1}{m}} \mathrm{~d} \xi_{1} \\
& \leq L_{m}^{(1)} L_{m}^{(2)} \int_{\Omega_{x_{1}}} \int_{\Omega_{x_{2}}}\left|H\left(\xi_{1}, \xi_{2}, x_{3} \ldots, x_{d}\right)\right|^{2+\frac{n-2}{m}} \mathrm{~d} \xi_{1} \mathrm{~d} \xi_{2} \\
& \leq L_{m}^{(1)} \cdots L_{m}^{(n)} \int_{\Omega_{x_{1}}} \cdots \int_{\Omega_{x_{d}}}\left|H\left(\xi_{1}, \ldots, \xi_{d}\right)\right|^{2} \mathrm{~d} \xi_{1} \cdots \mathrm{d} \xi_{n} .
\end{aligned}
$$

The constants $L_{m}^{(i)}, i=1, \ldots, n$, only depend on $m$,

$$
\left\|H\left(x_{1}, \ldots, x_{i-1}, \cdot, x_{i+1}, \ldots, x_{n}\right)\right\|_{L^{\infty}(\mathbb{R})} \leq\|H\|_{L^{\infty}\left(\mathbb{R}^{n}\right)}
$$

and

$$
\left\|\partial_{i}^{m} H\left(x_{1}, \ldots, x_{i-1}, \cdot, x_{i+1}, \ldots, x_{n}\right)\right\|_{L^{\infty}(\mathbb{R})} \leq\left\|D^{m} H\right\|_{L^{\infty}\left(\mathbb{R}^{n}\right)} .
$$

Setting $L_{m, n}=\prod_{i=1}^{n} L_{m}^{(i)}$ yields the stated inequality with $Q_{x}=\Omega_{x_{1}} \times \cdots \times \Omega_{x_{n}}$.

Remark 2.17. It is worth noticing that the exponent in Corollary 2.15 is decreasing in the dimension and increasing in $m$.

For the proof of Lemma 2.14 we need the following interpolation result between $L^{\infty}$ norms of derivatives of a function.

Lemma 2.18 (Kolmogorov-Landau inequality on the unit interval). Let $m \geq 2$ be an integer. There exists a constant $C_{m}>0$ such that for all $w \in W^{m, \infty}([0,1])$,

$$
\left\|w^{(k)}\right\|_{L^{\infty}([0,1])} \leq C_{m}\left(\frac{\|w\|_{L^{\infty}([0,1])}}{u^{k}}+u^{m-k}\left\|w^{(m)}\right\|_{L^{\infty}([0,1])}\right), \quad k=1, \ldots, m-1,
$$

for all $0<u \leq 1$.

Proof. The result dates back to E. Landau and A. N. Kolmogorov who proved it on $\mathbb{R}$ and $\mathbb{R}^{+}$. A proof of the inequality on a finite interval can be found in the book by R. A. DeVore and G. G. LORENTZ [17] (pp.37-39), but for the reader's convenience we also give a short proof in Appendix C.

For us, the important consequence we are going to make use of is

Corollary 2.19. Let $C_{m}>0$ be the constant from Lemma 2.18. Then for all $w \in W^{m, \infty}([0,1])$,

$$
\left\|w^{(k)}\right\|_{L^{\infty}([0,1])} \leq 2 C_{m}\|w\|_{L^{\infty}([0,1])}^{1-k / m} \max \left\{\|w\|_{L^{\infty}([0,1])}^{k / m},\left\|w^{(m)}\right\|_{L^{\infty}([0,1])}^{k / m}\right\}, \quad k=1, \ldots, m-1 .
$$

Proof. If $\left\|w^{(m)}\right\|_{L^{\infty}([0,1])} \leq\|w\|_{L^{\infty}([0,1])}$, we choose $u=1$ in the bound from Lemma 2.18, which gives

$$
\left\|w^{(k)}\right\|_{L^{\infty}([0,1])} \leq 2 C_{m}\|w\|_{L^{\infty}([0,1])}
$$

in this case, and if $\left\|w^{(m)}\right\|_{L^{\infty}([0,1])} \geq\|w\|_{L^{\infty}([0,1])}$, we can choose $u=\|w\|_{L^{\infty}([0,1])}^{1 / m}\left\|w^{(m)}\right\|_{L^{\infty}([0,1])}^{-1 / m} \leq 1$ to obtain

$$
\left\|w^{(k)}\right\|_{L^{\infty}([0,1])} \leq 2 C_{m}\|w\|_{L^{\infty}([0,1])}^{1-k / m}\left\|w^{(m)}\right\|_{L^{\infty}([0,1])}^{k / m} .
$$

Together this proves (30).

We can now turn to the 
Proof of Lemma 2.14. Assume without loss of generality that $r \geq 0$, so that $\Omega_{r}=[r, r+2]$. By the Sobolev embedding theorem $h$ is continuous and we let $r^{*}$ be a point in $\Omega_{r}$ where $|h|$ attains its maximum. We can assume that $r^{*} \in[r, r+1]$ and set $\langle h\rangle_{r^{*}}:=\int_{r^{*}}^{r^{*}+1} h(\xi) \mathrm{d} \xi$ (otherwise we use $\left.\langle h\rangle_{r^{*}}:=\int_{r^{*}-1}^{r^{*}} h(\xi) \mathrm{d} \xi\right)$. Then for some $p \geq 1$ we have

$$
\left|h\left(r^{*}\right)\right|^{p}-\left|\left\langle h^{p}\right\rangle_{r^{*}}\right| \leq \int_{r^{*}}^{r^{*}+1}\left|h^{p}\left(r^{*}\right)-h^{p}(\xi)\right| \mathrm{d} \xi=\int_{0}^{1}\left|h^{p}\left(r^{*}\right)-h^{p}\left(r^{*}+\zeta\right)\right| \mathrm{d} \zeta .
$$

Bt the fundamental theorem of calculus, for any $\zeta \in[0,1]$ the integrand can be bounded by

$$
\begin{aligned}
\left|h^{p}\left(r^{*}\right)-h^{p}\left(r^{*}+\zeta\right)\right| & \leq p \int_{0}^{1}\left|h\left(r^{*}+s \zeta\right)\right|^{p-1}\left|h^{\prime}\left(r^{*}+s \zeta\right)\right| \zeta \mathrm{d} s \\
& \leq p \sup _{s \in[0,1]}\left|h^{\prime}\left(r^{*}+s \zeta\right)\right| \int_{0}^{1}\left|h\left(r^{*}+s \zeta\right)\right|^{p-1} \zeta \mathrm{d} s
\end{aligned}
$$

We now use that

$$
\sup _{s \in[0,1]}\left|h^{\prime}\left(r^{*}+s \zeta\right)\right|=\sup _{x \in[0, \zeta]}\left|h^{\prime}\left(r^{*}+x\right)\right| \leq \sup _{x \in[0,1]}\left|h^{\prime}\left(r^{*}+x\right)\right|=\left\|h^{\prime}\left(r^{*}+\cdot\right)\right\|_{L^{\infty}([0,1])}
$$

and apply the Kolmogorov-Landau inequality for the first derivative in its multiplicative form from Corollary 2.19 to the function $[0,1] \ni x \mapsto h\left(r^{*}+x\right) \in W^{m, \infty}([0,1])$ to obtain

$$
\begin{aligned}
\left\|h^{\prime}\left(r^{*}+\cdot\right)\right\|_{L^{\infty}([0,1])} & \leq 2 C_{m}\left\|h\left(r^{*}+\cdot\right)\right\|_{L^{\infty}([0,1])}^{1-1 / m} \max \left\{\left\|h\left(r^{*}+\cdot\right)\right\|_{L^{\infty}([0,1])}^{1 / m},\left\|h^{(m)}\left(r^{*}+\cdot\right)\right\|_{L^{\infty}([0,1])}^{1 / m}\right\} \\
& \leq 2 C_{m}\left|h\left(r^{*}\right)\right|^{1-1 / m} \max \left\{\|h\|_{L^{\infty}(\mathbb{R})}^{1 / m},\left\|h^{(m)}\right\|_{L^{\infty}(\mathbb{R})}^{1 / m}\right\} .
\end{aligned}
$$

It follows that

$$
\begin{gathered}
\left|h\left(r^{*}\right)\right|^{p}-\left|\left\langle h^{p}\right\rangle_{r^{*}}\right| \leq 2 p C_{m}\left|h\left(r^{*}\right)\right|^{1-1 / m} \max \left\{\|h\|_{L^{\infty}(\mathbb{R})}^{1 / m},\left\|h^{(m)}\right\|_{L^{\infty}(\mathbb{R})}^{1 / m}\right\} \\
\times \int_{0}^{1} \int_{0}^{1}\left|h\left(r^{*}+s \zeta\right)\right|^{p-1} \zeta \mathrm{d} s \mathrm{~d} \zeta .
\end{gathered}
$$

The latter integral can be further estimated by

$$
\begin{aligned}
\int_{0}^{1} \int_{0}^{1}\left|h\left(r^{*}+s \zeta\right)\right|^{p-1} \zeta \mathrm{d} s \mathrm{~d} \zeta & =\int_{0}^{1} \int_{0}^{\zeta}\left|h\left(r^{*}+x\right)\right|^{p-1} \mathrm{~d} x \mathrm{~d} \zeta \\
& \leq \int_{0}^{1} \int_{0}^{1}\left|h\left(r^{*}+x\right)\right|^{p-1} \mathrm{~d} \zeta \mathrm{d} x=\int_{0}^{1}\left|h\left(r^{*}+x\right)\right|^{p-1} \mathrm{~d} x \\
& =\int_{r^{*}}^{r^{*}+1}|h(\xi)|^{p-1} \mathrm{~d} \xi \leq \int_{\Omega_{r}}|h(\xi)|^{p-1} \mathrm{~d} \xi .
\end{aligned}
$$

Using

$$
\begin{aligned}
\left|\left\langle h^{p}\right\rangle_{r^{*}}\right| \leq \int_{r^{*}}^{r^{*}+1}|h(\xi)|^{p} \mathrm{~d} \xi & \leq\|h\|_{L^{\infty}\left(\Omega_{r}\right)} \int_{\Omega_{r}}|h(\xi)|^{p-1} \mathrm{~d} \xi \\
& \leq\left|h\left(r^{*}\right)\right|^{1-1 / m}\|h\|_{L^{\infty}(\mathbb{R})}^{1 / m} \int_{\Omega_{r}}|h(\xi)|^{p-1} \mathrm{~d} \xi
\end{aligned}
$$

we get

$$
\left|h\left(r^{*}\right)\right|^{p} \leq L_{m}\left|h\left(r^{*}\right)\right|^{1-1 / m} \int_{\Omega_{r}}|h(\xi)|^{p-1} \mathrm{~d} \xi
$$

with $L_{m}=2 p C_{m} \max \left\{\|h\|_{L^{\infty}(\mathbb{R})}^{1 / m},\left\|h^{(m)}\right\|_{L^{\infty}(\mathbb{R})}^{1 / m}\right\}+\|h\|_{L^{\infty}(\mathbb{R})}^{1 / m}$, and therefore

$$
\left|h\left(r^{*}\right)\right|^{p-1+1 / m} \leq L_{m} \int_{\Omega_{r}}|h(\xi)|^{p-1} \mathrm{~d} \xi .
$$


Choosing $q:=p-1+1 / m \geq 1 / m$ then yields

$$
|h(r)|^{q} \leq\left|h\left(r^{*}\right)\right|^{q} \leq L_{m} \int_{\Omega_{r}}|h(\xi)|^{q-1 / m} \mathrm{~d} \xi,
$$

which is the claimed inequality.

2.4. Gevrey smoothing of weak solutions for $L^{2}$ initial data: Part I. Equipped with Corollary 2.15 we can construct an inductive scheme based upon a uniform bound on $G\left(\eta^{-}\right)^{\epsilon(\alpha, 1)}\left|\hat{f}\left(\eta^{-}\right)\right|$. As already remarked, this result will depend on the dimension, and will actually deteriorate quickly as dimension increases. Nevertheless it leads to strong regularity properties of weak solutions in the physically relevant cases.

Theorem 2.20. Assume that the initial datum $f_{0}$ satisfies $f_{0} \geq 0, f_{0} \in L \log L\left(\mathbb{R}^{d}\right) \cap L_{m}^{1}\left(\mathbb{R}^{d}\right)$ for some $m \geq 2$, and, in addition, $f_{0} \in L^{2}\left(\mathbb{R}^{d}\right)$. Further assume that the cross-section $b$ satisfies the singularity condition (3) and the integrability condition (4) for $d \geq 2$, and for $d=1, b_{1}$ satisfies the singularity condition (6) and the integrability condition (7) for some $0<v<1$. Let $f$ be a weak solution of the Cauchy problem (1) with initial datum $f_{0}$. Set $\alpha_{m, d}:=\log \left(\frac{4 m+d}{2 m+d}\right) / \log 2$. Then, for all $0<\alpha \leq \min \left\{\alpha_{m, d}, v\right\}$ and $T_{0}>0$, there exists $\beta>0$, such that for all $t \in\left[0, T_{0}\right]$

$$
e^{\beta t\left\langle D_{v}\right\rangle^{2 \alpha}} f(t, \cdot) \in L^{2}\left(\mathbb{R}^{d}\right),
$$

that is, $f \in G^{\frac{1}{2 \alpha}}\left(\mathbb{R}^{d}\right)$ for all $t \in\left(0, T_{0}\right]$.

By decreasing $\beta$, if necessary, one even has a uniform bound,

Corollary 2.21. Let $T_{0}>0$. Under the same conditions as in Theorem 2.20 there exit $\beta>0$ and $M_{1}<\infty$ such that

$$
\sup _{0 \leq t \leq T_{0}} \sup _{\eta \in \mathbb{R}^{d}} e^{\beta t\langle\eta\rangle^{2 \alpha}}|\hat{f}(t, \eta)| \leq M_{1} .
$$

Remark 2.22. (i) For strong singularities, the restriction on the Gevrey class originates in the bound on the commutation error, with the best value in $d=1$ dimension. The aim of part II below will be to recover the two-dimensional result in any dimension $d \geq 2$. Under slightly stronger assumptions on the angular cross-section, which still covers all physically relevant cases, we can get the one-dimensional result in any dimension $d \geq 1$, see part III.

(ii) In dimensions $d=1,2,3$ and $m=2$, corresponding to initial data with finite energy, we have $\alpha_{2, d}=\log \left(\frac{8+d}{4+d}\right) / \log 2 \geq \log \left(\frac{11}{7}\right) / \log 2 \simeq 0.652077$. This means that for $v=\frac{1}{2}$ the weak solution gets analytic and even ultra-analytic for $v>\frac{1}{2}$.

(iii) In the case of physical Maxwellian molecules, where $v=\frac{1}{4}$, in three dimensions and with initial datum having finite mass, energy and entropy, we obtain Gevrey $G^{2}\left(\mathbb{R}^{3}\right)$ regularity.

(iv) Even though the range of $\alpha$ in Theorem 2.20 above deteriorates as the dimension increases, it only fails to cover (ultra-)analyticity results in dimensions $d \geq 6$. Theorems 2.30 and 2.35 below yield results uniformly in the dimension.

We will prove Theorem 2.20 inductively over suitable length scales $\Lambda_{N} \rightarrow \infty$ as $N \rightarrow \infty$ in Fourier space. To prepare for this, we fix some $M<\infty, 0<T_{0}<\infty$ and introduce

Definition 2.23 (Hypothesis $\operatorname{Hyp}_{\Lambda}(M)$ ). Let $M \geq 0$. Then for all $0 \leq t \leq T_{0}$

$$
\sup _{|\zeta| \leq \Lambda} G(t, \zeta)^{\epsilon(\alpha, 1)}|\hat{f}(t, \zeta)| \leq M .
$$

Remark 2.24. Recall that $G(t, \zeta)=e^{\beta t\langle\zeta\rangle^{\alpha}}$, that is, it depends on $\alpha, \beta$, and $t$, and also $f$ is a time dependent function, even though we suppress this dependence in our notation. Thus $\operatorname{Hyp}_{\Lambda}(M)$ also depends on the parameters in $G(t, \zeta)$ and on $M$ and $T_{0}$, which, for simplicity, we do not emphasise in our notation. We will later fix some $T_{0}>0$ and a suitable large enough $M$. The main reason why this is possible is that, since $\|\hat{f}\|_{L^{\infty}} \leq\|f\|_{L^{1}}=\left\|f_{0}\right\|_{L^{1}}<\infty$, for any $\Lambda, \beta, T_{0}>0$ the hypothesis 
$\operatorname{Hyp} 1_{\Lambda}(M)$ is true for large enough $M$ and even any $M>\left\|f_{0}\right\|_{L^{1}}$ is possible by choosing $\beta>0$ small enough.

A first step into the inductive proof is the following

Lemma 2.25. Let $\alpha \leq v$ and define $c_{b, d}:=\left|\mathbb{S}^{d-2}\right| \int_{0}^{\frac{\pi}{2}} \sin ^{d} \theta b(\cos \theta) \mathrm{d} \theta$ for $d \geq 3, c_{b, 2}:=\int_{0}^{\frac{\pi}{2}} \sin ^{2} \theta b(\cos \theta) \mathrm{d} \theta$, $c_{b, 1}:=\int_{-\frac{\pi}{4}}^{\frac{\pi}{4}} \sin ^{2} \theta b_{1}(\theta) \mathrm{d} \theta$, which are finite by the integrability assumptions (4) and (7), and let $\beta \leq \frac{\tilde{C}_{f_{0}}}{\left(1+2^{d-1}\right) c_{b, d} \alpha T_{0} M+1}$. Then, for any weak solution of the homogenous Boltzmann equation,

$$
\operatorname{Hyp}_{\Lambda}(M) \quad \Rightarrow \quad\left\|G_{\sqrt{2} \Lambda} f\right\|_{L^{2}\left(\mathbb{R}^{d}\right)} \leq\left\|\mathbb{1}_{\sqrt{2} \Lambda}\left(D_{v}\right) f_{0}\right\|_{L^{2}\left(\mathbb{R}^{d}\right)} e^{C_{f_{0}} T_{0}}
$$

for all $0 \leq t \leq T_{0}$.

Remark 2.26. The main point of this lemma is that the right hand side of (34) does not depend on $M$. This is crucial for our analysis and might seem a bit surprising, at first. It is achieved by making $\beta$ small enough.

Proof. Let $d \geq 2$. Since $\cot ^{2} \frac{\theta}{2} \geq 1$ for $\theta \in\left[0, \frac{\pi}{2}\right]$ and $\cot ^{2} \vartheta \geq 1$ for $\vartheta \in\left[0, \frac{\pi}{4}\right]$, we can bound $\epsilon\left(\alpha, \cot ^{2} \frac{\theta}{2}\right)$ and $\epsilon\left(\alpha, \cot ^{2} \vartheta\right)$ by $\epsilon(\alpha, 1)$ in the integrals $I_{d, \sqrt{2} \Lambda}$ and $I_{d, \sqrt{2} \Lambda}^{+}$from Lemma 2.11 .

Assume Hyp $1_{\Lambda}(M)$ holds. Then

$$
G(t, \zeta)^{\epsilon(\alpha, 1)}|\hat{f}(t, \zeta)| \leq M \quad \text { for all }|\zeta| \leq \Lambda .
$$

In particular, the terms containing $\eta^{-}$in $I_{d, \sqrt{2} \Lambda}$ and $I_{d, \sqrt{2} \Lambda}^{+}$can be bounded by $M$. Thus, these integrals can now be further estimated by

$$
\begin{aligned}
I_{d, \sqrt{2} \Lambda} & \leq \alpha \beta t M\left|\mathbb{S}^{d-2}\right| \int_{0}^{\frac{\pi}{2}} \sin ^{d} \theta b(\cos \theta) \mathrm{d} \theta \int_{\mathbb{R}^{d}}\left|G_{\sqrt{2} \Lambda}(\eta) \hat{f}(\eta)\right|^{2}\langle\eta\rangle^{2 \alpha} \mathrm{d} \eta \\
& =\alpha \beta t M c_{b, d}\left\|G_{\sqrt{2} \Lambda} f\right\|_{H^{\alpha}\left(\mathbb{R}^{d}\right)}^{2}
\end{aligned}
$$

and,

$$
I_{d, \sqrt{2} \Lambda}^{+} \leq 2^{d} \alpha \beta t M\left|\mathbb{S}^{d-2}\right| \int_{0}^{\frac{\pi}{4}} \sin ^{d} \vartheta b(\cos 2 \vartheta) \mathrm{d} \vartheta \int_{\mathbb{R}^{d}}\left|G\left(\eta^{+}\right) \hat{f}\left(\eta^{+}\right)\right|^{2}\left\langle\eta^{+}\right\rangle^{2 \alpha} \mathrm{d} \eta^{+} .
$$

In the $\vartheta$ integral, we bound $\sin \vartheta \leq \sin (2 \vartheta)$ to obtain

$$
I_{d, \sqrt{2} \Lambda}^{+} \leq 2^{d-1} \alpha \beta t M c_{b, d}\left\|G_{\sqrt{2} \Lambda} f\right\|_{H^{\alpha}\left(\mathbb{R}^{d}\right)}^{2}
$$

By Lemma 2.11, the commutation error corresponding to the weight $G_{\sqrt{2} \Lambda}$ is thus bounded by

$$
\begin{aligned}
\left|\left\langle Q\left(f, G_{\sqrt{2} \Lambda} f\right)-G_{\sqrt{2} \Lambda} Q(f, f), G_{\sqrt{2} \Lambda} f\right\rangle\right| & \leq I_{d, \sqrt{2} \Lambda}+I_{d, \sqrt{2} \Lambda}^{+} \\
& \leq\left(1+2^{d-1}\right) \alpha \beta t M c_{b, d}\left\|G_{\sqrt{2} \Lambda} f\right\|_{H^{\alpha}\left(\mathbb{R}^{d}\right)}^{2} .
\end{aligned}
$$

With Corollary 2.4 we then have

$$
\begin{aligned}
\left\|G_{\sqrt{2} \Lambda} f\right\|_{L^{2}\left(\mathbb{R}^{d}\right)}^{2} \leq\left\|\mathbb{1}_{\sqrt{2} \Lambda}\left(D_{v}\right) f_{0}\right\|_{L^{2}}^{2}+\int_{0}^{t} 2 C_{f_{0}}\left\|G_{\sqrt{2} \Lambda} f\right\|_{L^{2}\left(\mathbb{R}^{d}\right)}^{2} \mathrm{~d} \tau \\
+\int_{0}^{t} 2\left(-\widetilde{C}_{f_{0}}\left\|G_{\sqrt{2} \Lambda} f\right\|_{H^{v}\left(\mathbb{R}^{d}\right)}^{2}+\left(\left(1+2^{d-1}\right) \alpha \beta t M c_{b, d}+\beta\right)\left\|G_{\sqrt{2} \Lambda} f\right\|_{H^{\alpha}\left(\mathbb{R}^{d}\right)}^{2}\right) \mathrm{d} \tau .
\end{aligned}
$$

Since $\alpha \leq v$ and $\beta \leq \frac{\widetilde{C}_{f_{0}}}{\left(1+2^{d-1}\right) c_{b, d} \alpha T_{0} M+1}$, this implies

$$
\left\|G_{\sqrt{2} \Lambda} f\right\|_{L^{2}\left(\mathbb{R}^{d}\right)}^{2} \leq\left\|\mathbb{1}_{\sqrt{2} \Lambda}\left(D_{v}\right) f_{0}\right\|_{L^{2}\left(\mathbb{R}^{d}\right)}^{2}+\int_{0}^{t} 2 C_{f_{0}}\left\|G_{\sqrt{2} \Lambda} f\right\|_{L^{2}\left(\mathbb{R}^{d}\right)}^{2} \mathrm{~d} \tau
$$

and with Gronwall's inequality

$$
\left\|G_{\sqrt{2} \Lambda} f\right\|_{L^{2}\left(\mathbb{R}^{d}\right)}^{2} \leq\left\|\mathbb{1}_{\sqrt{2} \Lambda}\left(D_{v}\right) f_{0}\right\|_{L^{2}\left(\mathbb{R}^{d}\right)}^{2} e^{2 C_{f_{0}} T_{0}}
$$


follows.

For $d=1$, we note that, with the obvious change in notation, the above proof literally translates to the Kac equation.

The second ingredient gives a uniform bound in terms of a weighted $L^{2}$ norm and some a priori uniform bound on some higher derivative of $\hat{f}$.

Lemma 2.27. Assume that there exist finite constants $A_{m}$ and $B$, such that

$$
\|f(t, \cdot)\|_{L_{m}^{1}} \leq A_{m}, \quad \text { and } \quad\left\|\left(G_{\sqrt{2} \Lambda} f\right)(t, \cdot)\right\|_{L^{2}\left(\mathbb{R}^{d}\right)} \leq B
$$

for some integer $m \geq 2$ and for all $0 \leq t \leq T_{0}$. Set

$$
\widetilde{\Lambda}:=\frac{1+\sqrt{2}}{2} \Lambda
$$

and assume furthermore that

Then for all $|\eta| \leq \widetilde{\Lambda}$

$$
\Lambda \geq \Lambda_{0}:=\frac{4 \sqrt{d}}{\sqrt{2}-1}
$$

$$
|\hat{f}(t, \eta)| \leq K_{1} G(t, \eta)^{-\frac{2 m}{2 m+d}} \quad \text { for all } \quad 0 \leq t \leq T_{0}
$$

with a constant $K_{1}$ depending only on the dimension $d, m, A_{m}$, and $B$.

Remark 2.28. The exponent $\frac{2 m}{2 m+d}$ in equation (40) comes from Corollary 2.15, choosing $n=d$. This is responsible for our definition of $\alpha_{m, d}$, since then $\epsilon\left(\alpha_{m, d}, 1\right)=\frac{2 m}{2 m+d}$.

Remark 2.29. The assumptions of Lemma 2.27 are quite natural: since the Boltzmann equation conserves mass and kinetic energy does not increase, we have the a priori estimate

$$
\|f(t, \cdot)\|_{L_{2}^{1}\left(\mathbb{R}^{d}\right)} \leq\left\|f_{0}\right\|_{L_{2}^{1}\left(\mathbb{R}^{d}\right)}=: A_{2},
$$

and due to the known results on moment propagation ${ }^{3}$ for the homogeneous Boltzmann equation in the Maxwellian molecules case, we have

$$
f_{0} \in L_{m}^{1}\left(\mathbb{R}^{d}\right) \Longrightarrow f(t, \cdot) \in L_{m}^{1}\left(\mathbb{R}^{d}\right) \text { uniformly in } t \geq 0
$$

for any $m>2$ in addition to assumptions (8).

The importance of Lemma 2.27 is that it effectively converts a local $L^{2}$ bound on suitable balls into a pointwise bound on slightly smaller balls.

Proof of Lemma 2.27. By the Riemann-Lebesgue lemma $\hat{f}$ has continuous and bounded derivatives of order up to $m$. Since for any multi-index $\alpha \in \mathbb{N}_{0}^{d}$ one has $\partial^{\alpha} \hat{f}=(-2 \pi \mathrm{i})^{|\alpha|} \widehat{v^{\alpha} f}$, we obtain the bound

$$
\begin{aligned}
\left\|D^{m} \hat{f}(t, \cdot)\right\|_{L^{\infty}\left(\mathbb{R}^{d}\right)} & =\sup _{\omega \in \mathbb{S}^{d-1}}\left\|(\omega \cdot \nabla)^{m} \hat{f}(t, \cdot)\right\|_{L^{\infty}\left(\mathbb{R}^{d}\right)} \leq \sup _{\omega \in \mathbb{S}^{d-1}} \sup _{\eta \in \mathbb{R}^{d}} \sum_{|\alpha|=m}\left(\begin{array}{c}
m \\
\alpha
\end{array}\right)\left|\omega^{\alpha}\right|\left|\partial^{\alpha} \hat{f}(\eta)\right| \\
& \leq(2 \pi)^{m} \sup _{\omega \in \mathbb{S}^{d-1}} \int_{\mathbb{R}^{d}} \sum_{|\alpha|=m}\left(\begin{array}{c}
m \\
\alpha
\end{array}\right)\left|\omega^{\alpha} v^{\alpha}\right| f(v) \mathrm{d} v \leq(2 \pi)^{m} \sup _{\omega \in \mathbb{S}^{d-1}} \int_{\mathbb{R}^{d}}(\omega \cdot v)^{m} f(v) \mathrm{d} v \\
& \leq(2 \pi)^{m} \int_{\mathbb{R}^{d}}|v|^{m} f(v) \mathrm{d} v \leq(2 \pi)^{m}\|f(t, \cdot)\|_{L_{m}^{1}\left(\mathbb{R}^{d}\right)} \leq(2 \pi)^{m} A_{m}
\end{aligned}
$$

Of course, also $\|\hat{f}\|_{L^{\infty}\left(\mathbb{R}^{d}\right)} \leq\|f\|_{L^{1}\left(\mathbb{R}^{d}\right)} \leq A_{m}$.

Let $\eta \in \mathbb{R}^{d}$ such that $|\eta| \leq \widetilde{\Lambda}$. By Corollary 2.15 applied to the function $\hat{f}$, there is a constant $L_{m, d}$ that depends only on $d, m$, and $A_{m}$ such that

$$
|\hat{f}(\eta)| \leq L_{m, d}\left(\int_{Q_{\eta}}|\hat{f}(\zeta)|^{2} \mathrm{~d} \zeta\right)^{\frac{m}{2 m+d}}
$$

\footnotetext{
${ }^{3}$ see, for instance, VillanI's review [40] pp. 73ff for references.
} 
where $Q_{\eta}$ is the cube of side length 2 at $\eta$, such that all sides are oriented away from the origin. The definitions of $\widetilde{\Lambda}$ and $\Lambda_{0}$ guarantee by Pythagoras' theorem, that, for $|\eta| \leq \widetilde{\Lambda}, Q_{\eta}$ always stays inside the ball around the origin with radius $\sqrt{2} \Lambda$. Since the orientation of $Q_{\eta}$ is such that $\eta$ is the point closest to the origin and the weight $G$ is radial and increasing, we have

$$
\begin{aligned}
|\hat{f}(\eta)| & \leq L_{m, d}\left(G(\eta)^{-2} \int_{Q_{\eta}} G(\zeta)^{2}|\hat{f}(\zeta)|^{2} \mathrm{~d} \zeta\right)^{\frac{m}{2 m+d}} \\
& \leq L_{m, d} G(\eta)^{-\frac{2 m}{2 m+d}}\left(\int_{\{|\eta| \leq \sqrt{2} \Lambda\}} G(\zeta)^{2}|\hat{f}(\zeta)|^{2} \mathrm{~d} \zeta\right)^{\frac{m}{2 m+d}} \\
& \leq L_{m, d} B^{\frac{2 m}{2 m+d}} G(\eta)^{-\frac{2 m}{2 m+d}} .
\end{aligned}
$$

Setting $K_{1}:=L_{m, d} B^{\frac{2 m}{2 m+d}}$ yields the claimed inequality.

Proof of Theorem 2.20. By Lemma 2.25, 2.27, and Remark 2.29, a suitable choice for $A_{m}, B$, and the length scales $\Lambda_{N}$ is

$$
\begin{aligned}
B & :=\left\|f_{0}\right\|_{L^{2}\left(\mathbb{R}^{d}\right)} e^{C_{f_{0}} T_{0}}, \\
A_{m} & :=\sup _{t \geq 0}\|f(t, \cdot)\|_{L_{m}^{1}\left(\mathbb{R}^{d}\right)}<\infty,
\end{aligned}
$$

and

$$
\Lambda_{N}:=\frac{\Lambda_{N-1}+\sqrt{2} \Lambda_{N-1}}{2}=\frac{1+\sqrt{2}}{2} \Lambda_{N-1}=\left(\frac{1+\sqrt{2}}{2}\right)^{N} \Lambda_{0}
$$

with $\Lambda_{0}$ from (39).

Furthermore, we set

$$
M_{1}:=\max \left\{2 A_{m}+1, K_{1}\right\}
$$

with the constant $K_{1}$ from equation (40).

For the start of the induction, we need $\operatorname{Hyp} 1_{\Lambda_{0}}\left(M_{1}\right)$ to be true. Since

$$
\sup _{0 \leq t \leq T_{0}} \sup _{|\eta| \leq \Lambda_{0}} G(\eta)^{\epsilon(\alpha, 1)}|\hat{f}(\eta)| \leq e^{\epsilon(\alpha, 1) \beta T_{0}\left(1+\Lambda_{0}^{2}\right)^{\alpha}} A_{m}
$$

and from our choice of $M_{1}$ there exists $\beta_{0}>0$ such that $\operatorname{Hyp}_{\Lambda_{0}}\left(M_{1}\right)$ is true for all $0 \leq \beta \leq \beta_{0}$.

Now, we choose

$$
\beta=\min \left(\beta_{0}, \frac{\tilde{C}_{f_{0}}}{\left(1+2^{d-1}\right) c_{b, d} \alpha T_{0} M_{1}+1}\right) .
$$

With this choice, the conditions of Lemma 2.25 and 2.27 are fulfilled and $\operatorname{Hyp}_{\Lambda_{0}}\left(M_{1}\right)$ is true.

For the induction step assume that $\operatorname{Hyp}_{\Lambda_{N}}\left(M_{1}\right)$ is true. Then Lemma 2.25 gives

$$
\left\|G_{\sqrt{2} \Lambda_{N}} f\right\|_{L^{2}\left(\mathbb{R}^{d}\right)} \leq\left\|\mathbb{1}_{\sqrt{2} \Lambda}\left(D_{v}\right) f_{0}\right\|_{L^{2}\left(\mathbb{R}^{d}\right)} e^{C_{f_{0}} T_{0}} \leq B .
$$

Note that $\epsilon(\alpha, 1) \leq \frac{2 m}{2 m+d}$, since $\alpha \leq \min \left\{\alpha_{m, d}, v\right\}$, see Remark 2.28. In addition, $\Lambda_{N+1}=\widetilde{\Lambda}_{N}$, so Lemma 2.27 shows

$$
\sup _{|\eta| \leq \Lambda_{N+1}} G(\eta)^{\epsilon(\alpha, 1)}|\hat{f}(\eta)| \leq K_{1} \leq M_{1}
$$

that is, $\operatorname{Hyp}_{\Lambda_{N+1}}\left(M_{1}\right)$ is true. By induction, it is true for all $N \in \mathbb{N}$. Invoking Lemma 2.25 again, we also have

$$
\left\|G_{\sqrt{2} \Lambda_{N}} f\right\|_{L^{2}\left(\mathbb{R}^{d}\right)} \leq B
$$

for all $N \in \mathbb{N}$ and passing to the limit $N \rightarrow \infty$, we see $\|G f\|_{L^{2}\left(\mathbb{R}^{d}\right)} \leq B$, which concludes the proof of the theorem.

Proof of Corollary 2.21. The proof of Theorem 2.20 showed that given $T_{0}>0$ there exists $M_{1}>0$ and $\beta>0$ such that $\operatorname{Hyp}_{\Lambda_{N}}\left(M_{1}\right)$ is true for all $N \in \mathbb{N}$. This clearly implies (32). 
2.5. Gevrey smoothing of weak solutions for $L^{2}$ initial data: Part II. The results of Part I are best in one dimension and give the correct smoothing in terms of the Gevrey class for $v$ not too close to one, more precisely $v \leq \alpha_{m, d}$. In order to improve this in higher dimensions $d \geq 2$ and for a larger range of singularities $0<v<1$, the commutator estimates have to be refined. We have

Theorem 2.30. Let $d \geq 3$. Assume that the initial datum $f_{0}$ satisfies $f_{0} \geq 0, f_{0} \in L \log L\left(\mathbb{R}^{d}\right) \cap$ $L_{m}^{1}\left(\mathbb{R}^{d}\right)$ for some $m \geq 2$, and, in addition, $f_{0} \in L^{2}\left(\mathbb{R}^{d}\right)$. Further assume that the cross-section $b$ satisfies the singularity condition (3) and the integrability condition (4) for some $0<v<1$. Let $f$ be a weak solution of the Cauchy problem (1) with initial datum $f_{0}$, then for all $0<\alpha \leq \min \left\{\alpha_{m, 2}, \nu\right\}$ and $T_{0}>0$, there exists $\beta>0$, such that for all $t \in\left[0, T_{0}\right]$

$$
e^{\beta t\left\langle D_{v}\right\rangle^{2 \alpha}} f(t, \cdot) \in L^{2}\left(\mathbb{R}^{d}\right),
$$

that is, $f \in G^{\frac{1}{2 \alpha}}\left(\mathbb{R}^{d}\right)$ for all $t \in\left(0, T_{0}\right]$.

In particular, the weak solution is real analytic if $v=\frac{1}{2}$ and ultra-analytic if $v>\frac{1}{2}$.

The beauty of this theorem is that, in contrast to Theorem 2.20 , its result does not deteriorate as dimension increases. We also have a corollary similar to Corollary 2.21 , however with a weaker conclusion. Moreover, it is not uniform in the time $t \geq 0$ but only holds on finite, but arbitrary, time intervals $\left[0, T_{0}\right]$.

Corollary 2.31. Under the same assumptions as in Theorem 2.30, for any weak solution $f$ of the Cauchy problem (1) and any $0<T_{0}<\infty$ there exists $\widetilde{\beta}>0$ and $M<\infty$ such that

$$
\sup _{0 \leq t \leq T_{0}} \sup _{\eta \in \mathbb{R}^{d}} e^{\widetilde{\beta} t}(\eta\rangle^{2 \alpha}|\hat{f}(t, \eta)| \leq M .
$$

The proof of Theorem 2.30 is again based on an induction over length scales in Fourier space. Having a close look at the integrals $I_{d, \Lambda}$ and $I_{d, \Lambda}^{+}$from Lemma 2.11 and using that $\epsilon(\alpha, \gamma)$ is decreasing in $\gamma$, one sees that it should be enough to bound expressions of the form

$$
\int_{\mathbb{S}^{d-2}(\eta)} G\left(\eta^{-}\right)^{\epsilon(\alpha, 1)}\left|\hat{f}\left(\eta^{-}\right)\right| \mathbb{1}_{\frac{\Lambda}{\sqrt{2}}}\left(\left|\eta^{-}\right|\right) \mathrm{d} \omega
$$

and

$$
\int_{\mathbb{S}^{d-2}\left(\eta^{+}\right)} G\left(\eta^{-}\right)^{\epsilon(\alpha, 1)}\left|\hat{f}\left(\eta^{-}\right)\right| \mathbb{1} \frac{\Lambda}{\sqrt{2}}\left(\left|\eta^{-}\right|\right) \mathrm{d} \omega
$$

uniformly in $\eta$ and $\theta$, respectively $\eta^{+}$and $\vartheta$, with the parametrization (25), respectively (28), that is, instead of having to use the purely pointwise estimates expressed in the hypothesis Hyp $1_{\Lambda}$ from the previous section, one can take advantage of averaging over codimension 2 spheres first. This motivates

Definition 2.32 (Hypothesis $\operatorname{Hyp}_{\Lambda}(\mathrm{M})$ ). Let $M \geq 0$ be finite. Then for all $0 \leq t \leq T_{0}$,

$$
\sup _{\zeta \in \mathbb{R}^{d} \backslash\{0\}} \sup _{(z, \rho) \in A_{\Lambda}} \int_{\mathbb{S}^{d-2}(\zeta)} G\left(t, z \frac{\zeta}{|\zeta|}-\rho \omega\right)^{\epsilon(\alpha, 1)}\left|\hat{f}\left(t, z \frac{\zeta}{|\zeta|}-\rho \omega\right)\right| \mathrm{d} \omega \leq M,
$$

where $A_{\Lambda}=\left\{(z, \rho) \in \mathbb{R}^{2}: 0 \leq z \leq \rho, z^{2}+\rho^{2} \leq \Lambda^{2}\right\}$ and $\mathbb{S}^{d-2}(\zeta)=\left\{\omega \in \mathbb{R}^{d}: \omega \perp \zeta,|\omega|=1\right\}$.

Again, we have

Lemma 2.33. Let $\alpha \leq v$, define $c_{b, d, 2}=\int_{0}^{\frac{\pi}{2}} \sin ^{d} \theta b(\cos \theta) \mathrm{d} \theta$ (which is finite by the integrability assumption (4)), and let $\beta \leq \frac{\tilde{C}_{f_{0}}}{\left(1+2^{d-1}\right) c_{b, d, 2} \alpha T_{0} M+1}$. Then, for any weak solution of the homogenous Boltzmann equation,

$$
\left(\operatorname{Hyp} 2_{\Lambda}\right) \quad \Rightarrow \quad\left\|G_{\sqrt{2} \Lambda} f\right\|_{L^{2}\left(\mathbb{R}^{d}\right)} \leq\left\|\mathbb{1}_{\sqrt{2} \Lambda}\left(D_{v}\right) f_{0}\right\|_{L^{2}\left(\mathbb{R}^{d}\right)} e^{C_{f_{0}} T_{0}}
$$

for all $0 \leq t \leq T_{0}$. 
Proof. Using the monotonicity of $\epsilon(\alpha, \gamma)$ in $\gamma$ and (24) one sees

$$
\begin{gathered}
I_{d, \sqrt{2} \Lambda} \leq \alpha \beta t \int_{\mathbb{R}^{d}}\left(\int_{0}^{\frac{\pi}{2}} \sin ^{d} \theta b(\cos \theta)\left(\int_{\mathbb{S}^{d-2}(\eta)} G\left(\eta^{-}\right)^{\epsilon(\alpha, 1)}\left|\hat{f}\left(\eta^{-}\right)\right| \mathbb{1}_{\Lambda}\left(\left|\eta^{-}\right|\right) \mathrm{d} \omega\right) \mathrm{d} \theta\right) \\
\times\left|G_{\sqrt{2} \Lambda}(\eta) \hat{f}(\eta)\right|^{2}\langle\eta\rangle^{2 \alpha} \mathrm{d} \eta
\end{gathered}
$$

where $\eta^{-}=\eta^{-}(\eta, \theta, \omega)$ is expressed via the parametrization (25). For $\sigma=(\theta, \omega) \in\left[0, \frac{\pi}{2}\right] \times \mathbb{S}^{d-2}$, one has $\eta^{-}=|\eta| \sin ^{2} \frac{\theta}{2} \frac{\eta}{|\eta|}+|\eta| \sin \frac{\theta}{2} \cos \frac{\theta}{2} \omega$ and if $|\eta| \leq \sqrt{2} \Lambda$, then $\left|\eta^{-}\right| \leq \Lambda$. Identifying $z=|\eta| \sin ^{2} \frac{\theta}{2}$ and $\rho=|\eta| \sin \frac{\theta}{2} \cos \frac{\theta}{2}$, and the direction of $\zeta$ with the direction of $\eta$, hypothesis $\left(\operatorname{Hyp}_{\Lambda}\right)$ clearly implies

$$
\sup _{|\eta| \leq \sqrt{2} \Lambda} \sup _{\theta \in[0, \pi / 2]} \int_{\mathbb{S}^{d-2}(\eta)} G\left(\eta^{-}\right)^{\epsilon(\alpha, 1)}\left|\hat{f}\left(\eta^{-}\right)\right| \mathbb{1}_{\Lambda}\left(\left|\eta^{-}\right|\right) \mathrm{d} \omega \leq M
$$

It follows that

$$
\begin{aligned}
I_{d, \sqrt{2} \Lambda} & \leq \alpha \beta t M \int_{\mathbb{R}^{d}} \int_{0}^{\frac{\pi}{2}} \sin ^{d} \theta b(\cos \theta) \mathrm{d} \theta\left|G_{\sqrt{2} \Lambda}(\eta) \hat{f}(\eta)\right|^{2}\langle\eta\rangle \mathrm{d} \eta \\
& =\alpha \beta t M c_{b, d, 2}\left\|G_{\sqrt{2} \Lambda} f\right\|_{H^{\alpha}\left(\mathbb{R}^{d}\right)}^{2} .
\end{aligned}
$$

Similarly one has

$$
\begin{aligned}
I_{d, \sqrt{2} \Lambda}^{+} \leq 2^{d} \alpha \beta t \int_{\mathbb{R}^{d}}\left(\int_{0}^{\frac{\pi}{4}} \sin ^{d} \vartheta b(\cos 2 \vartheta)\left(\int_{\mathbb{S}^{d-2}\left(\eta^{+}\right)} G\left(\eta^{-}\right)^{\epsilon(\alpha, 1)}\left|\hat{f}\left(\eta^{-}\right)\right| \mathbb{1}_{\Lambda}\left(\left|\eta^{-}\right|\right) \mathrm{d} \omega\right) \mathrm{d} \vartheta\right) \\
\times\left|G_{\sqrt{2} \Lambda}\left(\eta^{+}\right) \hat{f}\left(\eta^{+}\right)\right|^{2}\left\langle\eta^{+}\right\rangle^{2 \alpha} \mathrm{d} \eta^{+}
\end{aligned}
$$

where $\eta^{-}=\eta^{-}(\eta, \vartheta, \omega)$ is expressed via the parametrization (28). The vectors $\eta^{-}$and $\eta^{+}$are orthogonal and we have $\eta^{-}=-\left|\eta^{+}\right| \tan \vartheta \omega$ for $(\vartheta, \omega) \in\left[0, \frac{\pi}{4}\right] \times \mathbb{S}^{d-2}\left(\eta^{+}\right)$.

Setting $z=0$ and $\rho=\left|\eta^{+}\right| \tan \vartheta$ we have $\rho=\left|\eta^{-}\right| \leq \Lambda$ in the $\vartheta$ and $\eta^{+}$integrals above. Thus $\left(\operatorname{Hyp} 2_{\Lambda}\right)$ again implies

$$
\sup _{\left|\eta^{+}\right| \leq \sqrt{2} \Lambda} \sup _{\vartheta \in[0, \pi / 4]} \int_{\mathbb{S}^{d-2}\left(\eta^{+}\right)} G\left(\eta^{-}\right)^{\epsilon(\alpha, 1)}\left|\hat{f}\left(\eta^{-}\right)\right| \mathbb{1}_{\Lambda}\left(\left|\eta^{-}\right|\right) \mathrm{d} \omega \leq M
$$

Hence,

$$
\begin{aligned}
I_{d, \sqrt{2} \Lambda}^{+} & \leq 2^{d} \alpha \beta t M \int_{0}^{\frac{\pi}{2}} \sin ^{d} \theta b(\cos \theta) \mathrm{d} \theta \int_{\mathbb{R}^{d}}\left|G_{\sqrt{2} \Lambda}\left(\eta^{+}\right) \hat{f}\left(\eta^{+}\right)\right|^{2}\left\langle\eta^{+}\right\rangle \mathrm{d} \eta^{+} \\
& \leq 2^{d-1} \alpha \beta t M c_{b, d, 2}\left\|G_{\sqrt{2} \Lambda} f\right\|_{H^{\alpha}\left(\mathbb{R}^{d}\right)^{.}}^{2}
\end{aligned}
$$

The rest of the proof is the same as in the proof of Lemma 2.25.

To close the induction process, we next show

Lemma 2.34. Let $\beta \leq \frac{1}{T_{0}}$. Assume that there exist finite constants $A_{m}$ and $B$, such that

$$
\|f(t, \cdot)\|_{L_{m}^{1}} \leq A_{m}, \quad \text { and } \quad\left\|\left(G_{\sqrt{2} \Lambda} f\right)(t, \cdot)\right\|_{L^{2}\left(\mathbb{R}^{d}\right)} \leq B
$$

for some integer $m \geq 2$ and for all $0 \leq t \leq T_{0}$.

Set $\widetilde{\Lambda}:=\frac{1+\sqrt{2}}{2} \Lambda$ and assume that

$$
\Lambda \geq \Lambda_{0}:=\frac{4 \sqrt{2}}{\sqrt{2}-1} .
$$

Then for all $\zeta \in \mathbb{R}^{d} \backslash\{0\}$ and $0 \leq z \leq \rho$ with $\rho^{2}+z^{2} \leq \widetilde{\Lambda}^{2}$ one has

$$
\int_{\mathbb{S}^{d-2}(\zeta)}\left|\hat{f}\left(t, z \frac{\zeta}{|\zeta|}+\rho \omega\right)\right| \mathrm{d} \omega \leq K_{2} \widetilde{G}\left(t, z^{2}+\rho^{2}\right)^{-\frac{2 m}{2 m+2}} \quad \text { for all } 0 \leq t \leq T_{0}
$$

with a constant $K_{2}$ depending only on $d, m, A_{m}$, and B. Recall that $\widetilde{G}(t, s)=e^{\beta t(1+s)^{\alpha}}$. 
Proof. Fix $0<t \leq T_{0}, \zeta \in \mathbb{R}^{d} \backslash\{0\}$, and set $F(\rho, z):=\hat{f}\left(t, z \frac{\zeta}{\zeta \zeta}+\rho \omega\right)$, where we drop, for simplicity, the dependence on the time $t$ in our notation for $F$. Then, since $\|f(t, \cdot)\|_{L_{m}^{1}} \leq A_{m}$ one has $\hat{f}(t, \cdot) \in$ $\mathscr{C}^{m}\left(\mathbb{R}^{d}\right)$ and thus also $F \in \mathscr{C}^{m}\left(\mathbb{R}^{2}\right)$ with $\|F\|_{L^{\infty}} \leq A_{m}\left\|\partial_{\rho}^{m} F\right\|_{L^{\infty}} \leq(2 \pi)^{m} A_{m}$, and $\left\|\partial_{z}^{m} F\right\|_{L^{\infty}} \leq(2 \pi)^{m} A_{m}$ and Corollary 2.15 applied to $F$ yields

$$
\left|\hat{f}\left(z \frac{\zeta}{\zeta \zeta \mid}+\rho \omega\right)\right| \leq L_{m, 2}\left(\int_{\rho}^{\rho+2} \int_{z}^{z+2}\left|\hat{f}\left(x \frac{\zeta}{|\zeta|}+y \omega\right)\right|^{2} \mathrm{~d} x \mathrm{~d} y\right)^{\frac{m}{2 m+2}} .
$$

where we also dropped the dependence of $\hat{f}$ on the time variable $t$. Furthermore, we will drop the time dependence of $G$ and $\widetilde{G}$ in the following, that is, $G(\xi)$ and $\widetilde{G}(s)$ will stand for $G(t, \xi)$, respectively $\widetilde{G}(t, s)$.

To recover the $L^{2}$ norm of $G_{\sqrt{2} \Lambda} f$ in the right hand side of (47) we now need to take care of three things:

(i) Multiply with a suitable power of the radially increasing weight $G$.

(ii) Integrate over the missing $d-2$ directions, which will be taken care of by integrating over $\mathbb{S}^{d-2}(\zeta)$ and taking into account additional factors to get the $d$-dimensional Lebesgue measure.

(iii) Ensure that the region of integration $[\rho, \rho+2] \times[z, z+2] \times \mathbb{S}^{d-2}(\zeta)$ stays inside a ball of radius $\sqrt{2} \Lambda$ uniformly in the direction of $\zeta$. This we control by choosing $\Lambda_{0}$ large enough (a simple geometric consideration shows that $\Lambda_{0}$ from the statement of Lemma 2.34 works) and restricting $\rho$ and $z$ by $\rho^{2}+z^{2} \leq \widetilde{\Lambda}^{2}$.

Let $z, \rho \geq 0$. In the region of integration in (47), the point $\rho \omega+z \frac{\eta}{\eta \eta}$ is closest to the origin in $\mathbb{R}^{d}$, and since the weight $G$ is radially increasing, we get

$$
\begin{aligned}
\left|\hat{f}\left(z \frac{\zeta}{|\zeta|}+\rho \omega\right)\right| \leq L_{m, 2} \widetilde{G}\left(z^{2}+\rho^{2}\right)^{-\frac{2 m}{2 m+2}} & \left(\int_{\rho}^{\rho+2} \int_{z}^{z+2} G\left(x \frac{\zeta}{|\zeta|}+y \omega\right)^{2}\left|\hat{f}\left(x \frac{\zeta}{|\zeta|}+y \omega\right)\right|^{2} \mathrm{~d} x \mathrm{~d} y\right)^{\frac{m}{2 m+2}} .
\end{aligned}
$$

Assume that $z^{2}+\rho^{2} \leq \widetilde{\Lambda}^{2}$. Then the integration of inequality (48) over $\mathbb{S}^{d-2}(\zeta)$ yields with an application of Jensen's inequality ( $t \mapsto t \frac{m}{2 m+2}$ is concave!)

$$
\begin{aligned}
\int_{\mathbb{S}^{d-2}(\zeta)} \mid \hat{f} & \left.\left(z \frac{\zeta}{|\zeta|}+\rho \omega\right)\left|\mathrm{d} \omega \leq L_{m, 2}\right| \mathbb{S}^{d-2}\right|^{\frac{m+2}{2 m+2}} \widetilde{G}\left(z^{2}+\rho^{2}\right)^{-\frac{2 m}{2 m+2}} \\
& \times\left(\int_{\mathbb{S}^{d-2}(\zeta)} \int_{\rho}^{\rho+2} \int_{z}^{z+2} G_{\sqrt{2} \Lambda}\left(x \frac{\zeta}{|\zeta|}+y \omega\right)^{2}\left|\hat{f}\left(x \frac{\eta}{|\eta|}+y \omega\right)\right|^{2} \mathrm{~d} x \mathrm{~d} y \mathrm{~d} \omega\right)^{\frac{m}{2 m+2}} .
\end{aligned}
$$

Now assume additionally $0 \leq z \leq \rho$ and $\Lambda_{0}^{2} \leq \rho^{2}+z^{2} \leq \widetilde{\Lambda}^{2}$. Since $0 \leq z \leq \rho$ we have $\Lambda_{0}^{2} \leq z^{2}+\rho^{2} \leq$ $2 \rho^{2}$ and therefore

$$
\begin{aligned}
& \int_{\mathbb{S}^{d-2}(\zeta)} \int_{\rho}^{\rho+2} \int_{z}^{z+2} G_{\sqrt{2} \Lambda}\left(x \frac{\zeta}{|\zeta|}+y \omega\right)^{2}\left|\hat{f}\left(x \frac{\zeta}{|\zeta|}+y \omega\right)\right|^{2} \mathrm{~d} x \mathrm{~d} y \mathrm{~d} \omega \\
& \leq 2^{\frac{d-2}{2}} \Lambda_{0}^{2-d} \int_{\mathbb{S}^{d-2}(\zeta)} \int_{\rho}^{\rho+2} \int_{z}^{z+2} G_{\sqrt{2} \Lambda}\left(x \frac{\zeta}{|\zeta|}+y \omega\right)^{2}\left|\hat{f}\left(x \frac{\zeta}{|\zeta|}+y \omega\right)\right|^{2} y^{d-2} \mathrm{~d} x \mathrm{~d} y \mathrm{~d} \omega \\
& \leq 2^{\frac{d-2}{2}} \Lambda_{0}^{2-d}\left\|G_{\sqrt{2} \Lambda} f\right\|_{L^{2}\left(\mathbb{R}^{d}\right)}^{2},
\end{aligned}
$$

since $y^{d-2} \mathrm{~d} x \mathrm{~d} y \mathrm{~d} \omega$ is the $d$-dimensional Lebesgue measure in the cylindrical coordinates $(x, y \omega)$ with $x \in \mathbb{R}, y>0, \omega \in \mathbb{S}^{d-2}(\zeta)$ along the cylinder with axis $\zeta$. So with the assumption $\left\|G_{\sqrt{2} \Lambda} f\right\|_{L^{2}\left(\mathbb{R}^{d}\right)} \leq$ $B$ we obtain

$$
\int_{\mathbb{S}^{d-2}(\zeta)}\left|\hat{f}\left(t, z \frac{\zeta}{|\zeta|}+\rho \omega\right)\right| \mathrm{d} \omega \leq L_{m, 2}\left|\mathbb{S}^{d-2}\right|^{\frac{m+2}{2 m+2}}\left(2^{\frac{d-2}{2}} \Lambda_{0}^{2-d} B^{2}\right)^{\frac{m}{2 m+2}} \widetilde{G}\left(t, z^{2}+\rho^{2}\right)^{-\frac{2 m}{2 m+2}} .
$$


In the case $z^{2}+\rho^{2} \leq \Lambda_{0}^{2}$ we have $\widetilde{G}\left(t, z^{2}+\rho^{2}\right)^{-1} e^{\beta t\left(1+\Lambda_{0}^{2}\right)^{\alpha}} \geq 1$ and we can simply bound

$$
\begin{aligned}
\int_{\mathbb{S}^{d-2}(\zeta)}\left|\hat{f}\left(t, z \frac{\zeta}{|\zeta|}+\rho \omega\right)\right| \mathrm{d} \omega & \leq \widetilde{G}\left(t, z^{2}+\rho^{2}\right)^{-\frac{2 m}{2 m+2}} e^{\frac{2 m}{2 m+2} \beta t\left(1+\Lambda_{0}^{2}\right)^{\alpha}}\left|\mathbb{S}^{d-2}\right|\|\hat{f}(t, \cdot)\|_{L^{\infty}\left(\mathbb{R}^{d}\right)} \\
& \leq A_{m}\left|\mathbb{S}^{d-2}\right| e^{1+\Lambda_{0}^{2}} \widetilde{G}\left(t, z^{2}+\rho^{2}\right)^{-\frac{2 m}{2 m+2}}
\end{aligned}
$$

since $\beta \leq 1 / T_{0}$, by assumption. So choosing

$$
K_{2}:=\max \left(L_{m, 2}\left|\mathbb{S}^{d-2}\right|^{\frac{m+2}{2 m+2}}\left(2^{\frac{d-2}{2}} \Lambda_{0}^{2-d} B^{2}\right)^{\frac{m}{2 m+2}}, A_{m}\left|\mathbb{S}^{d-2}\right| e^{1+\Lambda_{0}^{2}}\right)
$$

finishes the proof of the lemma.

Now we have all the ingredients for the inductive

Proof of Theorem 2.30. By Lemmata 2.33 and 2.34 a suitable choice for $A_{m}$ and $B$ is

$$
\begin{aligned}
B & :=\left\|f_{0}\right\|_{L^{2}\left(\mathbb{R}^{d}\right)} e^{C_{f_{0}} T_{0}}, \\
A_{m} & :=\sup _{t \geq 0}\|f(t, \cdot)\|_{L_{m}^{1}\left(\mathbb{R}^{d}\right)}<\infty .
\end{aligned}
$$

Note that the finiteness of $A_{m}$ is guaranteed since $f_{0} \in L_{m}^{1}\left(\mathbb{R}^{d}\right)$, see Remark 2.29. We further choose the length scales $\Lambda_{N}$ to be

$$
\Lambda_{N}:=\frac{\Lambda_{N-1}+\sqrt{2} \Lambda_{N-1}}{2}=\frac{1+\sqrt{2}}{2} \Lambda_{N-1}=\left(\frac{1+\sqrt{2}}{2}\right)^{N} \Lambda_{0}
$$

with $\Lambda_{0}$ now from (46), and we set

$$
M_{2}:=\max \left\{2\left|\mathbb{S}^{d-2}\right| A_{m}+1, K_{2}\right\}
$$

with the constant $K_{2}$ from Lemma 2.34.

For the start of the induction, we need $\operatorname{Hyp} 2{ }_{\Lambda_{0}}\left(M_{2}\right)$ to be true. Since

$$
\begin{aligned}
\sup _{0 \leq t \leq T_{0}} \sup _{\zeta \in \mathbb{R}^{d} \backslash\{0\}} \sup _{(z, \rho) \in A_{\Lambda_{0}}} & \int_{\mathbb{S}^{d-2}(\zeta)} G\left(t, z \frac{\zeta}{|\zeta|}-\rho \omega\right)^{\epsilon(\alpha, 1)}\left|\hat{f}\left(t, z \frac{\zeta}{|\zeta|}-\rho \omega\right)\right| \mathrm{d} \omega \\
& \leq\left|\mathbb{S}^{d-2}\right| e^{\beta T_{0}\left(1+\Lambda_{0}^{2}\right)} A_{m}
\end{aligned}
$$

and from our choice of $M_{2}$ there exists $\beta_{0}>0$ such that $\operatorname{Hyp} 2_{\Lambda_{0}}\left(M_{2}\right)$ is true for all $0 \leq \beta \leq \beta_{0}$.

Now, we choose

$$
\beta=\min \left(\beta_{0}, T_{0}^{-1}, \frac{\tilde{C}_{f_{0}}}{\left(1+2^{d-1}\right) c_{b, d, 2} \alpha T_{0} M_{2}+1}\right) .
$$

With this choice, the conditions of Lemma 2.33 and 2.34 are fulfilled and $\operatorname{Hyp} 2{ }_{\Lambda_{0}}\left(M_{2}\right)$ is true.

For the induction step assume that $\operatorname{Hyp}_{\Lambda_{N}}\left(M_{2}\right)$ is true. Then Lemma 2.33 gives

$$
\left\|G_{\sqrt{2} \Lambda_{N}} f\right\|_{L^{2}\left(\mathbb{R}^{d}\right)} \leq\left\|\mathbb{1}_{\sqrt{2} \Lambda}\left(D_{v}\right) f_{0}\right\|_{L^{2}\left(\mathbb{R}^{d}\right)} e^{C_{f_{0}} T_{0}}=B
$$

and then, since $\epsilon(\alpha, 1) \leq \frac{2 m}{2 m+2}$ by our choice of $\alpha$, and $\Lambda_{N+1}=\widetilde{\Lambda}_{N}$, Lemma 2.34 shows that Hyp $2_{\Lambda_{N+1}}\left(M_{2}\right)$ is true, so by induction, it is true for all $N \in \mathbb{N}$. Invoking Lemma 2.33 again, we also have

$$
\left\|G_{\sqrt{2} \Lambda_{N}} f\right\|_{L^{2}\left(\mathbb{R}^{d}\right)} \leq B
$$

for all $N \in \mathbb{N}$ and letting $N \rightarrow \infty$, we see $\|G f\|_{L^{2}\left(\mathbb{R}^{d}\right)} \leq B$, which concludes the proof of Theorem 2.30 .

Proof of Corollary 2.31. Theorem 2.30 shows that $G f \in L^{2}\left(\mathbb{R}^{d}\right)$ for all $0 \leq t \leq T_{0}$. applying Corollary 2.15 with $n=d$ to $\hat{f}$ yields

$$
|\hat{f}(\eta)| \leq L_{m, d} G(\eta)^{-\frac{2 m}{2 m+d}}\left(\int_{Q_{\eta}} G(\zeta)^{2}|\hat{f}(\zeta)|^{2} \mathrm{~d} \zeta\right)^{\frac{m}{2 m+d}} \leq L_{m, d}\|G f\|_{L^{2}\left(\mathbb{R}^{d}\right)}^{\frac{2 m}{2 m+d}} G(\eta)^{-\frac{2 m}{2 m+d}}
$$


where we also used that the Fourier multiplier is radially increasing. This proves the uniform bound (42) with $\widetilde{\beta}=\beta \frac{2 m}{2 m+d}$.

2.6. Gevrey smoothing of weak solutions for $L^{2}$ initial data: Part III. Under the slightly stronger assumption on the angular collision cross-section $b$, namely that $b$ is bounded away from the singularity, we can state out theorem about Gevrey regularisation in its strongest form.

Theorem 2.35. Assume that the initial datum $f_{0}$ satisfies $f_{0} \geq 0, f_{0} \in L \log L\left(\mathbb{R}^{d}\right) \cap L_{m}^{1}\left(\mathbb{R}^{d}\right)$ for some $m \geq 2$, and, in addition, $f_{0} \in L^{2}\left(\mathbb{R}^{d}\right)$. Further assume that the cross-section $b$ in dimensions $d \geq 2$ satisfies the singularity condition (3) for some $0<v<1$ and the boundedness condition (16). Let $f$ be a weak solution of the Cauchy problem (1) with initial datum $f_{0}$, then for all $0<\alpha \leq \min \left\{\alpha^{m, 1}, v\right\}$ and all $T_{0}>0$, there exists $\beta>0$, such that for all $t \in\left[0, T_{0}\right]$

$$
e^{\beta t\left\langle D_{v}\right\rangle^{2 \alpha}} f(t, \cdot) \in L^{2}\left(\mathbb{R}^{d}\right),
$$

that is, $f \in G^{\frac{1}{2 \alpha}}\left(\mathbb{R}^{d}\right)$ for all $t \in\left(0, T_{0}\right]$.

In particular, the weak solution is real analytic if $v=\frac{1}{2}$ and ultra-analytic if $v>\frac{1}{2}$.

Remark 2.36. Thus, under slightly stronger assumption on $b$ than in Theorem 2.20, which we stress are nevertheless fulfilled in any physically reasonable cases, we can prove the same regularity in any dimension as can be obtained for radially symmetric solutions of the homogenous Boltzmann equation.

Corollary 2.37. Under the same assumptions as in Theorem 2.35, for any weak solution $f$ of the Cauchy problem (1) and any $0<T_{0}<\infty$ there exists $\beta>0$ and $M<\infty$ such that

$$
\sup _{0 \leq t \leq T_{0}} \sup _{\eta \in \mathbb{R}^{d}} e^{\beta t\langle\eta\rangle^{2 \alpha}}|\hat{f}(t, \eta)| \leq M .
$$

Proof. Given Theorem 2.35, the proof of Corollary 2.37 is the same as the proof of Corollary 2.31 .

The proof of Theorem 2.35 shows the delicate interplay between the angular singularity of the collision kernel, the strict concavity of the Gevrey weights, and the use of averages of the weak solution in Fourier space, together with our inductive procedure, which has proved to be successful in Theorems 2.20 and 2.30. Again, the main work is to bound the expressions $I_{d, \Lambda}$ and $I_{d, \Lambda}^{+}$from Lemma 2.11. Before we start the proof of Theorem 2.35, we start with some preparations. It is clear that we only have to prove Theorem 2.35 in dimension $d \geq 2$ and for singularities $v>\alpha_{2, m}$, since otherwise the result is already contained in Theorems 2.20 and 2.30.

Looking at the integral $I_{d, \Lambda}$ from Lemma 2.11, one has

$$
\begin{gathered}
I_{d, \Lambda}=\alpha \beta t \int_{\mathbb{R}^{d}}\left(\int_{0}^{\frac{\pi}{2}} \int_{\mathbb{S}^{d-2}(\eta)} \sin ^{d} \theta b(\cos \theta) G\left(\eta^{-}\right)^{\epsilon\left(\alpha, \cot ^{2} \frac{\theta}{2}\right)}\left|\hat{f}\left(\eta^{-}\right)\right| \mathbb{1}_{\frac{\Lambda}{\sqrt{2}}}\left(\left|\eta^{-}\right|\right) \mathrm{d} \omega \mathrm{d} \theta\right) \\
\times\left|G_{\Lambda}(\eta) \hat{f}(\eta)\right|^{2}\langle\eta\rangle^{2 \alpha} \mathrm{d} \eta .
\end{gathered}
$$

where we use the parametrization (25) for $\eta^{-}=\eta^{-}(\eta, \theta, \omega)$. Splitting the $\theta$ integral above at a point $\theta_{0} \in\left(0, \frac{\pi}{2}\right)$ and using the monotonicity of the cotangent on $\left[0, \frac{\pi}{2}\right]$ and of $\epsilon(\alpha, \gamma)$ in $\gamma$ one sees

$$
I_{d, \Lambda} \leq I_{d, \Lambda, 1}+I_{d, \Lambda, 2}
$$

whith

$$
\begin{aligned}
I_{d, \Lambda, 1}:=\alpha \beta T_{0} & \sup _{0<\theta \leq \frac{\pi}{2}} \sup _{0<|\eta| \leq \Lambda} \int_{\mathbb{S}^{d-2}(\eta)} G\left(\eta^{-}(\eta, \theta, \omega)\right)^{\epsilon\left(\alpha, \cot ^{2} \frac{\theta_{0}}{2}\right)}\left|\hat{f}\left(\eta^{-}(\eta, \theta, \omega)\right)\right| \mathbb{1}_{\frac{\Lambda}{\sqrt{2}}}\left(\left|\eta^{-}(\eta, \theta, \omega)\right|\right) \mathrm{d} \omega \\
& \times \int_{0}^{\theta_{0}} \sin ^{d} \theta b(\cos \theta) \mathrm{d} \theta\left\|G_{\Lambda} f\right\|_{H^{\alpha}\left(\mathbb{R}^{d}\right)}^{2}
\end{aligned}
$$


and

$$
\begin{aligned}
I_{d, \Lambda, 2}:= & C_{\theta_{0}} \alpha \beta T_{0} \sup _{0<|\eta| \leq \Lambda} \int_{\theta_{0}}^{\frac{\pi}{2}} \int_{\mathbb{S}^{d-2}(\eta)} G\left(\eta^{-}(\eta, \theta, \omega)\right)^{\epsilon(\alpha, 1)}\left|\hat{f}\left(\eta^{-}(\eta, \theta, \omega)\right)\right| \mathbb{1}_{\frac{\Lambda}{\sqrt{2}}}\left(\left|\eta^{-}(\eta, \theta, \omega)\right|\right) \mathrm{d} \omega \mathrm{d} \theta \\
& \times\left\|G_{\Lambda} f\right\|_{H^{\alpha}\left(\mathbb{R}^{d}\right)}^{2}
\end{aligned}
$$

where $C_{\theta_{0}}$ is an upper bound for $b(\cos \theta)$ on $\left[\theta_{0}, \frac{\pi}{2}\right]$. Now we choose $\theta_{0}>0$ so small that

$$
\epsilon\left(\alpha, \cot ^{2} \frac{\theta_{0}}{2}\right) \leq \epsilon\left(\alpha_{2, m}, 1\right)=\frac{2 m}{2 m+2}
$$

and note that from Corollary 2.31, since $v>\alpha_{2, m}$, there exists a finite $M_{2}$ such that

$$
\sup _{0<\theta \leq \frac{\pi}{2}} \sup _{0<|\eta| \leq \Lambda} \int_{\mathbb{S}^{d-2}(\eta)} G\left(\eta^{-}(\eta, \theta, \omega)\right)^{\epsilon\left(\alpha_{2, m}, 1\right)}\left|\hat{f}\left(\eta^{-}(\eta, \theta, \omega)\right)\right| \mathbb{1}_{\frac{\Lambda}{\sqrt{2}}}\left(\left|\eta^{-}(\eta, \theta, \omega)\right|\right) \mathrm{d} \omega \leq M_{2}<\infty .
$$

So from (51) we get the bound

$$
I_{d, \Lambda, 1} \leq \alpha \beta T_{0} M_{2} c_{b, d, 2}\left\|G_{\Lambda} f\right\|_{H^{\alpha}\left(\mathbb{R}^{d}\right)}^{2}
$$

where the finiteness of $c_{b, d, 2}$ follows from the singularity condition and the boundedness of $b(\cos \theta)$ away from $\theta=0$.

For the integral $I_{d, \Lambda}^{+}$from Lemma 2.11, a completely analogous reasoning as above shows for small enough $\vartheta_{0}$ such that $\epsilon\left(\alpha \cot ^{\vartheta}\right) \leq \epsilon\left(\alpha_{2, m}, 1\right)$ we also have

$$
I_{d, \Lambda}^{+} \leq I_{d, \Lambda, 1}^{+}+I_{d, \Lambda, 2}^{+}
$$

with

$$
I_{d, \Lambda, 1}^{+} \leq 2^{d-1} \alpha \beta T_{0} M_{2} c_{b, d, 2}\left\|G_{\Lambda} f\right\|_{H^{\alpha}\left(\mathbb{R}^{d}\right)}^{2}
$$

and

$$
\begin{aligned}
I_{d, \Lambda, 2}^{+}:= & 2^{d} C_{\vartheta_{0}} \alpha \beta T_{0} \sup _{0<\left|\eta^{+}\right| \leq \Lambda} \int_{\vartheta_{0}}^{\frac{\pi}{4}} \int_{\mathbb{S}^{d-2}\left(\eta^{+}\right)} G\left(\eta^{-}\left(\eta^{+}, \vartheta, \omega\right)\right)^{\epsilon(\alpha, 1)}\left|\hat{f}\left(\eta^{-}\left(\eta^{+}, \vartheta, \omega\right)\right)\right| \mathbb{1} \frac{\Lambda}{\sqrt{2}}\left(\left|\eta^{-}\left(\eta^{+}, \vartheta, \omega\right)\right|\right) \mathrm{d} \omega \mathrm{d} \vartheta \\
& \times\left\|G_{\Lambda} f\right\|_{H^{\alpha}\left(\mathbb{R}^{d}\right)}^{2}
\end{aligned}
$$

where we use the parametrization (28) for $\eta^{-}=\eta^{-}\left(\eta^{+}, \vartheta, \omega\right)$ and where $C_{\vartheta_{0}}$ is an upper bound for $b(\cos (2 \vartheta))$ on $\left[\vartheta_{0}, \frac{\pi}{4}\right]$.

Recall that we always assume $\alpha \leq \alpha_{1, m}$, so $\epsilon(\alpha, 1) \leq \epsilon\left(\alpha_{1, m}, 1\right)=\frac{2 m}{2 m+1}$. Thus we see that in order to set up our inductive procedure for controlling $I_{d \Lambda}$ and $I_{d, \Lambda}^{+}$it is natural to introduce

Definition 2.38 (Hypothesis $\mathbf{H y p 3}_{\Lambda}(M)$ ). Let $M \geq 0$ be finite, $0<\theta_{0}, \vartheta_{0}<\frac{\pi}{4}, T_{0}>0$, and $m \geq 2$ an integer. Then for all $0 \leq t \leq T_{0}$ one has

$$
\sup _{|\eta| \leq \sqrt{2} \Lambda} \int_{\theta_{0}}^{\frac{\pi}{2}} \int_{\mathbb{S}^{d-2}(\eta)} G\left(t, \eta^{-}(\eta, \theta, \omega)\right)^{\frac{2 m}{2 m+1}}\left|\hat{f}\left(\eta^{-}(\eta, \theta, \omega)\right)\right| \mathbb{1}_{\Lambda}\left(\left|\eta^{-}(\eta, \theta, \omega)\right|\right) \mathrm{d} \omega \mathrm{d} \theta \leq M,
$$

where we use the parametrization given in (25) for $\eta^{-}$, and

$$
\sup _{\left|\eta^{+}\right| \leq \sqrt{2} \Lambda} \int_{\vartheta_{0}}^{\frac{\pi}{4}} \int_{\mathbb{S}^{d-2}\left(\eta^{+}\right)} G\left(t, \eta^{-}\left(\eta^{+}, \vartheta, \omega\right)\right)^{\frac{2 m}{2 m+1}}\left|\hat{f}\left(\eta^{-}\left(\eta^{+}, \vartheta, \omega\right)\right)\right| \mathbb{1}_{\Lambda}\left(\left|\eta^{-}\left(\eta^{+}, \vartheta, \omega\right)\right|\right) \mathrm{d} \omega \mathrm{d} \vartheta \leq M
$$

where we use the parametrization given in (28) for $\eta^{-}$.

For the induction proof of Theorem 2.35, we again start with 
Lemma 2.39. Let $M \geq 0, T_{0}>0, m \geq 2$ an integer, $\alpha_{m, 2}<v<1,0<\alpha \leq v$ and recall $c_{b, d, 2}=\int_{0}^{\frac{\pi}{2}} \sin ^{d} \theta b(\cos \theta) \mathrm{d} \theta$ (which is finite by the singularity assumption (4) and the boundedness assumption (16)). Let $M_{2}$ be from Corollary 2.31 and $\beta \leq \frac{\tilde{C}_{f_{0}}}{\alpha T_{0}\left[\left(1+2^{d-1}\right) c_{b, d, 2} M_{2}+\left(C_{\theta_{0}}+2^{d} C_{\vartheta_{0}}\right) M\right]+1}$. Then for any weak solution of the homogenous Boltzmann equation,

$$
\mathbf{H y p 3}_{\Lambda}(M) \Rightarrow\left\|G_{\sqrt{2} \Lambda} f\right\|_{L^{2}\left(\mathbb{R}^{d}\right)} \leq\left\|\mathbb{1}_{\sqrt{2} \Lambda}\left(D_{v}\right) f_{0}\right\|_{L^{2}\left(\mathbb{R}^{d}\right)} e^{C_{f_{0}} T_{0}}
$$

for all $0 \leq t \leq T_{0}$.

Proof. Given Lemma 2.11 and the above discussion with the bounds in (53), (54) and using the hypotheses $\left(\operatorname{Hyp} 3_{\Lambda}\right)$ for the terms in (52) and (55), one sees that the commutation error on the level $\sqrt{2} \Lambda$ is bounded by

$$
\begin{aligned}
& \left|\left\langle Q\left(f, G_{\sqrt{2} \Lambda} f\right)-G_{\sqrt{2} \Lambda} Q(f, f), G_{\sqrt{2} \Lambda} f\right\rangle\right| \leq I_{d, \sqrt{2} \Lambda}+I_{d, \sqrt{2} \Lambda}^{+} \\
& \leq\left(1+2^{d-1}\right) \alpha \beta T_{0} M_{2} c_{b, d, 2}\left\|G_{\Lambda} f\right\|_{H^{\alpha}\left(\mathbb{R}^{d}\right)}^{2}+\left(C_{\theta_{0}}+2^{d} C_{\vartheta_{0}}\right) \alpha \beta T_{0} M\left\|G_{\Lambda} f\right\|_{H^{\alpha}\left(\mathbb{R}^{d}\right)}^{2}
\end{aligned}
$$

Given this bound on the commutation error, the rest of the proof is the same as in the proof of Lemma 2.25.

To close the induction step we also need a suitable version of Lemma 2.34 but before we prove this we need a preparatory Lemma.

Lemma 2.40. Let $H: \mathbb{R}^{d} \rightarrow \mathbb{R}_{+}$be a locally integrable function and let $\eta, \eta_{+} \in \mathbb{R}^{d}$ with $|\eta|,\left|\eta^{+}\right| \geq$ $\Lambda_{0}>0,0<\theta_{0} \leq \frac{\pi}{2}$, and $0<\vartheta_{0} \leq \frac{\pi}{8}$. Then with the parametrization $\eta^{-}=\eta^{-}(\eta, \theta, \omega)$ given in (25) one has

$$
\int_{\theta_{0}}^{\frac{\pi}{2}} \int_{0}^{2} H\left(\eta^{-}(\eta, \theta, \omega)+z \frac{\eta}{|\eta|}\right) \mathrm{d} z \mathrm{~d} \theta \leq \frac{2}{\Lambda_{0} \cos \theta_{0}} \int_{\Lambda_{0} \sin ^{2} \frac{\theta_{0}}{2}}^{\frac{|\eta|}{2}+2} \int_{\Lambda_{0} \sin \theta_{0}}^{\frac{|\eta|}{2}} H\left(x \frac{\eta}{|\eta|}-y \omega\right) \mathrm{d} y \mathrm{~d} x
$$

for any unit vector $\omega$ orthogonal to $\eta$. Moreover, with the parametrization $\eta^{-}=\eta^{-}\left(\eta^{+}, \theta, \omega\right)$ given in (28) one has, for any $\widetilde{\Lambda} \geq \frac{1+\sqrt{2}}{2} \Lambda_{0}$,

$$
\begin{aligned}
\int_{\vartheta_{0}}^{\frac{\pi}{4}} \int_{0}^{2} H\left(\eta^{-}\left(\eta^{+}, \vartheta, \omega\right)+z \frac{\eta}{|\eta|}\right) & \frac{\mathbb{\Lambda}_{\frac{\Lambda}{\sqrt{2}}}}{}\left(\left|\eta^{-}\left(\eta^{+}, \vartheta, \omega\right)\right|\right) \mathrm{d} z \mathrm{~d} \vartheta \\
& \leq \frac{1}{2 \Lambda_{0}} \int_{0}^{2} \int_{\Lambda_{0} \tan \vartheta_{0}}^{\frac{\tilde{\Lambda}}{\sqrt{2}}} H\left(x \frac{\eta}{|\eta|}-y \omega\right) \mathrm{d} y \mathrm{~d} x
\end{aligned}
$$

Remark 2.41. The restriction $\vartheta_{0} \leq \frac{\pi}{8}$ is only for convenience, to ensure that $\Lambda_{0} \tan \vartheta_{0} \leq \frac{\pi}{\sqrt{2}}$.

Proof. Fix $\eta$ as required and $\omega$ orthogonal to it. We want to have a map $\Phi_{1}:(\theta, z) \mapsto \Phi_{1}(\theta, z)=$ $(x, y)$ such that

$$
\eta^{-}(\eta, \theta, \omega)+z \frac{\eta}{|\eta|}=x \frac{\eta}{|\eta|}-y \omega
$$

From the parametrization (25) we read off

$$
x=|\eta| \sin ^{2} \frac{\theta}{2}+z \quad \text { and } y=\frac{|\eta|}{2} \sin \theta
$$

and we can compute the Jacobian going from the $(\theta, z)$ variables to $(x, y)$ as

$$
\left|\frac{\partial(x, y)}{\partial(\theta, z)}\right|=\left|\operatorname{det} D \Phi_{1}\right|=\frac{|\eta|}{2} \cos \theta \geq \frac{|\eta|}{2} \cos \theta_{0} .
$$

Since $|\eta| \geq \Lambda_{0}, \theta \in\left[\theta_{0}, \frac{\pi}{2}\right]$, and $0 \leq z \leq 2$, we have $\Lambda_{0} \sin ^{2} \frac{\theta_{0}}{2} \leq x \leq|\eta| \sin ^{2} \frac{\pi}{4}=\frac{\eta}{2}$ and $\frac{\Lambda_{0}}{2} \sin \theta_{0} \leq$ $y \leq \frac{\eta}{2}$. So doing a change of variables $(\theta, z)=\Phi_{1}^{-1}(x, y)$ in the integral we can bound

$$
\int_{\theta_{0}}^{\frac{\pi}{2}} \int_{0}^{2} H\left(\eta^{-}(\eta, \theta, \omega)+z \frac{\eta}{|\eta|}\right) \mathrm{d} z \mathrm{~d} \theta \leq \frac{2}{\Lambda_{0} \cos \theta_{0}} \int_{\Lambda_{0} \sin ^{2} \frac{\theta_{0}}{2}}^{\frac{|\eta|}{2}+2} \int_{\Lambda_{0} \sin \theta_{0}}^{\frac{|\eta|}{2}} H\left(x \frac{\eta}{|\eta|}+y \omega\right) \mathrm{d} y \mathrm{~d} x
$$


since the map $\Phi_{1}$ is a nice diffeomorphism.

For the second bound the calculation is, in fact, a bit easier, one just has to take care that $\left|\eta^{-}\right|$ cannot be too large, which is taken into account by the factor $\mathbb{1}_{\Lambda}\left(\left|\eta^{-}\right|\right)$. We now want a map $\Phi_{2}$ : $(\theta, z) \mapsto \Phi_{2}(\theta, z)=(x, y)$ such that

$$
\eta^{-}\left(\eta^{+}, \vartheta, \omega\right)+z \frac{\eta^{+}}{\left|\eta^{+}\right|}=x \frac{\eta^{+}}{\left|\eta^{+}\right|}-y \omega .
$$

From the parametrization (25) we read off

$$
x=z \quad \text { and } y=\left|\eta^{-}\right|=\left|\eta^{+}\right| \tan \vartheta
$$

and the Jacobian going from the $(\vartheta, z)$ variables to $(x, y)$ is simply

$$
\left|\frac{\partial(x, y)}{\partial(\vartheta, z)}\right|=\left|\operatorname{det} D \Phi_{2}\right|=2\left|\eta^{+}\right| \geq 2 \Lambda_{0} .
$$

We certainly have $0 \leq x \leq 2$ and also $\Lambda_{0} \tan \vartheta_{0} \leq y$. Since $y=\left|\eta^{-}\right|$, we also have the restriction $y \leq \Lambda$. So the proof of the second inequality follows similar to the proof of first one.

Finally, we can state and prove the second step in our inductive procedure.

Lemma 2.42. Let $\beta \leq \frac{1}{T_{0}}$. Asssume that there exist finite constants $A_{m}$ and $B$, such that

$$
\|f(t, \cdot)\|_{L_{m}^{1}} \leq A_{m}, \quad \text { and } \quad\left\|\left(G_{\sqrt{2} \Lambda} f\right)(t, \cdot)\right\|_{L^{2}\left(\mathbb{R}^{d}\right)} \leq B
$$

for some integer $m \geq 2$ and for all $0 \leq t \leq T_{0}$.

Set $\widetilde{\Lambda}:=\frac{1+\sqrt{2}}{2} \Lambda$ and assume that

$$
\Lambda \geq \Lambda_{0}:=3 .
$$

Then there exist a finite $K_{3}$, depending only on $d, m, A_{m}$, and $B$ such that $\operatorname{Hyp}_{\widetilde{\Lambda}}\left(K_{3}\right)$ is true.

Proof. Fix $0<t \leq T_{0}$, a direction $\eta \in \mathbb{R}^{d} \backslash\{0\}$, and define the function

$$
z \mapsto F(z):=\hat{f}\left(t, \eta^{-}+z \frac{\eta}{|\eta|}\right)
$$

of the single real variable $z$, where we think of $\eta^{-}$as given in the $\eta$-parametrization (25) for some $\theta$ and $\omega \in \mathbb{S}^{d-2}(\eta)$, and where we drop, for simplicity, the dependence on the time $t$ in our notation for $F$ and $f$. Then, since $\|f(t, \cdot)\|_{L_{m}^{1}} \leq A_{m}$ one has $\hat{f}(t, \cdot) \in \mathscr{C}^{m}\left(\mathbb{R}^{d}\right)$ and thus also $F \in \mathscr{C}^{m}(\mathbb{R})$ with $\|F\|_{L^{\infty}} \leq A_{m}\left\|\partial_{z}^{m} F\right\|_{L^{\infty}} \leq(2 \pi)^{m} A_{m}$, and Corollary 2.15 applied to $F$ now gives

$$
\left|\hat{f}\left(\eta^{-}\right)\right| \leq L_{m, 1}\left(\int_{0}^{2}\left|\hat{f}\left(\eta^{-}+z \frac{\eta}{|\eta|}\right)\right|^{2} \mathrm{~d} z\right)^{\frac{m}{2 m+2}} .
$$

We multiply this with the radially increasing weight $G$ to get

$$
G\left(\eta^{-}\right)^{\frac{2 m}{2 m+1}}\left|\hat{f}\left(\eta^{-}\right)\right| \leq L_{m, 1}\left(\int_{0}^{2}\left|G\left(\eta^{-}+z \frac{\eta}{|\eta|}\right) \hat{f}\left(\eta^{-}+z \frac{\eta}{|\eta|}\right)\right|^{2} \mathrm{~d} z\right)^{\frac{m}{2 m+2}} .
$$

Integrating this with respect to $\omega$ and $\theta$, where we think of $\eta^{-}=\eta^{-}(\eta, \theta, \omega)$ in the parametrization (25), and using Jensen's inequality for concave functions, one gets

$$
\begin{aligned}
& \int_{\theta_{0}}^{\frac{\pi}{2}} \int_{\mathbb{S}^{d-2}(\eta)} G\left(\eta^{-}\right)^{\frac{2 m}{2 m+1}}\left|\hat{f}\left(\eta^{-}\right)\right| \mathrm{d} \theta \mathrm{d} \omega \\
& \quad \leq L_{m, 1}\left(\frac{\pi}{2}\right)^{\frac{m+1}{2 m+1}\left|\mathbb{S}^{d-2}\right| \frac{m+1}{2 m+1}}\left(\int_{\theta_{0}}^{\frac{\pi}{2}} \int_{\mathbb{S}^{d-2}(\eta)} \int_{0}^{2}\left|G\left(\eta^{-}+z \frac{\eta}{|\eta|}\right) \hat{f}\left(\eta^{-}+z \frac{\eta}{|\eta|}\right)\right|^{2} \mathrm{~d} z \mathrm{~d} \theta \mathrm{d} \omega\right)^{\frac{m}{2 m+1}} .
\end{aligned}
$$


Now assume that $|\eta| \geq \Lambda_{0}$. Because of the first part of Lemma 2.40, we can further bound

$$
\begin{aligned}
(62) \leq & L_{m, 1}\left(\frac{\pi}{2}\right)^{\frac{m+1}{2 m+1}}\left|\mathbb{S}^{d-2}\right|^{\frac{m+1}{2 m+1}}\left(\frac{2}{\Lambda_{0} \cos \theta_{0}}\right)^{\frac{m}{2 m+1}} \\
& \left(\int_{\mathbb{S}^{d-2}(\eta)} \int_{\Lambda_{0} \sin ^{2} \frac{\theta_{0}}{2}}^{\frac{|\eta|}{2}+2} \int_{\Lambda_{0} \sin \theta_{0}}^{\frac{\eta \eta}{2}}\left|G\left(x \frac{\eta}{|\eta|}-y \omega\right) \hat{f}\left(x \frac{\eta}{|\eta|}-y \omega\right)\right|^{2} \mathrm{~d} y \mathrm{~d} x \mathrm{~d} \omega\right)^{\frac{m}{2 m+1}} \\
\leq & L_{m, 1}\left(\frac{\pi}{2}\right)^{\frac{m+1}{2 m+1}\left|\mathbb{S}^{d-2}\right|^{\frac{m+1}{2 m+1}}}\left(\frac{2}{\Lambda_{0} \cos \theta_{0}}\right)^{\frac{m}{2 m+1}}\left(\Lambda_{0} \sin \theta_{0}\right)^{2-d} \\
& \left(\int_{\mathbb{S}^{d-2}(\eta)} \int_{\Lambda_{0} \sin ^{2} \frac{\theta_{0}}{2}}^{\frac{\mid \eta \eta}{2}+2} \int_{\Lambda_{0} \sin \theta_{0}}^{\frac{|\eta|}{2}}\left|G\left(x \frac{\eta}{|\eta|}-y \omega\right) \hat{f}\left(x \frac{\eta}{|\eta|}-y \omega\right)\right|^{2} y^{d-2} \mathrm{~d} y \mathrm{~d} x \mathrm{~d} \omega\right)^{\frac{m}{2 m+1}}
\end{aligned}
$$

Again, the integration measure $y^{d-2} \mathrm{~d} y \mathrm{~d} x \mathrm{~d} \omega$ is $d$-dimensional Lebesgue measure in the cylindrical coordinates $(x, y \omega)$ with respect to the cylinder in the $\eta$ direction. One checks that the condition $\Lambda \geq \Lambda_{0} \geq 3$ ensures that

$$
(\widetilde{\Lambda} / 2+2)^{2}+(\widetilde{\Lambda} / 2) \leq(\sqrt{2} \Lambda)^{2}
$$

so since $|\eta| \leq \widetilde{\Lambda}$, we can extend the integration above to a ball of radius $\sqrt{2} \Lambda$ to get

$$
\begin{aligned}
& \text { (62) } \leq L_{m, 1}\left(\frac{\pi}{2}\right)^{\frac{m+1}{2 m+1}}\left|\mathbb{S}^{d-2}\right|^{\frac{m+1}{2 m+1}}\left(\frac{2}{\Lambda_{0} \cos \theta_{0}}\right)^{\frac{m}{2 m+1}}\left(\Lambda_{0} \sin \theta_{0}\right)^{2-d} \|\left. G_{\sqrt{2} \Lambda} f\right|_{L^{2}\left(\mathbb{R}^{d}\right)} ^{\frac{2 m}{2 m+1}} \\
& \leq L_{m, 1}\left(\frac{\pi}{2}\right)^{\frac{m+1}{2 m+1}}\left|\mathbb{S}^{d-2}\right|^{\frac{m+1}{2 m+1}}\left(\frac{2}{\Lambda_{0} \cos \theta_{0}}\right)^{\frac{m}{2 m+1}}\left(\Lambda_{0} \sin \theta_{0}\right)^{2-d} B^{\frac{2 m}{2 m+1}} .
\end{aligned}
$$

If $|\eta| \leq \Lambda_{0}$ we simply bound

$$
\int_{\theta_{0}}^{\frac{\pi}{2}} \int_{\mathbb{S}^{d-2}(\eta)} G\left(\eta^{-}\right)^{\frac{2 m}{2 m+1}}\left|\hat{f}\left(\eta^{-}\right)\right| \mathrm{d} \theta \mathrm{d} \omega \leq\|\hat{f}\|_{L^{\infty}} \frac{\pi}{2}\left|\mathbb{S}^{d-2}\right| e^{\beta T_{0}\left(1+\Lambda_{0}^{2} / 2\right)} \leq A_{m} \frac{\pi}{2}\left|\mathbb{S}^{d-2}\right| e^{1+\Lambda_{0}^{2} / 2} .
$$

Concerning the bound in the second half of $\mathrm{Hyp}_{\tilde{\Lambda}}$, a completely analogous calculation as the one above, using the second halft of Lemma 2.40 gives for $\lambda_{0} \leq\left|\eta^{+}\right| \leq \widetilde{\Lambda}$,

$$
\begin{aligned}
& \int_{\vartheta_{0}}^{\frac{\pi}{2}} \int_{\mathbb{S}^{d-2}\left(\eta^{+}\right)} G\left(t, \eta^{-}\left(\eta^{+}, \vartheta, \omega\right)\right)^{\frac{2 m}{2 m+1}}\left|\hat{f}\left(\eta^{-}\left(\eta^{+}, \vartheta, \omega\right)\right)\right| \mathbb{1}_{\frac{\Lambda}{\sqrt{2}}}\left(\left|\eta^{-}\left(\eta^{+}, \vartheta, \omega\right)\right|\right) \mathrm{d} \omega \mathrm{d} \vartheta \\
& \leq L_{m, 1}\left(\frac{\pi}{2}\right)^{\frac{m+1}{2 m+1}}\left|\mathbb{S}^{d-2}\right|^{\frac{m+1}{2 m+1}}\left(\frac{1}{2 \Lambda_{0}}\right)^{\frac{m}{2 m+1}}\left(\Lambda_{0} \tan \vartheta_{0}\right)^{2-d} \\
& \quad\left(\int_{\mathbb{S}^{d-2}\left(\eta^{+}\right)} \int_{0}^{2} \int_{0}^{\frac{\pi}{\sqrt{2}}}\left|G\left(x \frac{\eta}{|\eta|}-y \omega\right) \hat{f}\left(x \frac{\eta}{|\eta|}-y \omega\right)\right|^{2} y^{d-2} \mathrm{~d} y \mathrm{~d} x \mathrm{~d} \omega\right)^{\frac{m}{2 m+1}}
\end{aligned}
$$

By our choice of $\widetilde{\Lambda}$ and $\Lambda_{0}$, we always have $2^{2}+(\widetilde{\Lambda} / 2)^{2} \leq(\sqrt{2} \Lambda)^{2}$, so we can extend the integration above to the whole ball $\left|\eta^{+}\right| \leq \sqrt{2} \Lambda$ to see

$$
\begin{aligned}
(65) & \leq L_{m, 1}\left(\frac{\pi}{2}\right)^{\frac{m+1}{2 m+1}}\left|\mathbb{S}^{d-2}\right| \frac{m+1}{2 m+1}\left(\frac{1}{2 \Lambda_{0}}\right)^{\frac{m}{2 m+1}}\left(\Lambda_{0} \tan \vartheta_{0}\right)^{2-d} \|\left. G_{\sqrt{2} \Lambda} f\right|_{L^{2}\left(\mathbb{R}^{d}\right)} ^{\frac{2 m}{2 m+1}} \\
& \leq L_{m, 1}\left(\frac{\pi}{2}\right)^{\frac{m+1}{2 m+1}}\left|\mathbb{S}^{d-2}\right|^{\frac{m+1}{2 m+1}}\left(\frac{1}{2 \Lambda_{0}}\right)^{\frac{m}{2 m+1}}\left(\Lambda_{0} \tan \vartheta_{0}\right)^{2-d} B^{\frac{2 m}{2 m+1}}
\end{aligned}
$$

If $\left|\eta^{+}\right| \leq \Lambda_{0}$ we simply bound as above

$$
\int_{\vartheta_{0}}^{\frac{\pi}{4}} \int_{\mathbb{S}^{d-2}\left(\eta^{+}\right)} G\left(\eta^{-}\right)^{\frac{2 m}{2 m+1}}\left|\hat{f}\left(\eta^{-}\right)\right| \mathrm{d} \vartheta \mathrm{d} \omega \leq A_{m} \frac{\pi}{4}\left|\mathbb{S}^{d-2}\right| e^{1+\Lambda_{0}^{2}}
$$


Now we set $K_{3}$ equal to the maximum of the constants in (63), (64), (66), (67). with this choice, $K_{3}$ depends only on $d, m, A_{m}$, and $B$ and $\operatorname{Hyp} \widetilde{\Lambda}_{\Lambda}\left(K_{3}\right)$ is true.

Proof of Theorem 2.35. By Lemmata 2.33 and 2.34 a suitable choice for $A_{m}$ and $B$ is

$$
\begin{aligned}
B & :=\left\|f_{0}\right\|_{L^{2}\left(\mathbb{R}^{d}\right)} e^{C_{f_{0}} T_{0}}, \\
A_{m} & :=\sup _{t \geq 0}\|f(t, \cdot)\|_{L_{m}^{1}\left(\mathbb{R}^{d}\right)}<\infty .
\end{aligned}
$$

Note that the finiteness of $A_{m}$ is guaranteed since $f_{0} \in L_{m}^{1}\left(\mathbb{R}^{d}\right)$, see Remark 2.29. Again choose the length scales $\Lambda_{N}$ to be

$$
\Lambda_{N}:=\frac{\Lambda_{N-1}+\sqrt{2} \Lambda_{N-1}}{2}=\frac{1+\sqrt{2}}{2} \Lambda_{N-1}=\left(\frac{1+\sqrt{2}}{2}\right)^{N} \Lambda_{0}
$$

with $\Lambda_{0}=3$, see (61), and we set

$$
M_{3}:=\max \left\{2\left|\mathbb{S}^{d-2}\right| A_{m}+1, K_{3}\right\}
$$

with the constant $K_{3}$ from Lemma 2.42 .

For the start of the induction, we need $\operatorname{Hyp}_{\Lambda_{0}}\left(M_{3}\right)$ to be true. Since

$$
\begin{aligned}
\sup _{0 \leq t \leq T_{0}} \sup _{\zeta \in \mathbb{R}^{d} \backslash\{0\}} \sup _{(z, \rho) \in A_{\Lambda_{0}}} & \int_{\mathbb{S}^{d-2}(\zeta)} G\left(t, z \frac{\zeta}{|\zeta|}-\rho \omega\right)^{\epsilon(\alpha, 1)}\left|\hat{f}\left(t, z \frac{\zeta}{|\zeta|}-\rho \omega\right)\right| \mathrm{d} \omega \\
& \leq\left|\mathbb{S}^{d-2}\right| e^{\beta T_{0}\left(1+\Lambda_{0}^{2}\right)} A_{m}
\end{aligned}
$$

and from our choice of $M_{2}$ there exists $\beta_{0}>0$ such that Hyp2 ${ }_{\Lambda_{0}}\left(M_{2}\right)$ is true for all $0 \leq \beta \leq \beta_{0}$.

Now, we choose

$$
\beta=\min \left(\beta_{0}, T_{0}^{-1}, \frac{\tilde{C}_{f_{0}}}{2^{d} c_{b, d, 2} \alpha T_{0} M_{2}+1}\right) .
$$

With this choice, the conditions of Lemma 2.33 and 2.34 are fulfilled and $\operatorname{Hyp} 2{ }_{\Lambda_{0}}\left(M_{2}\right)$ is true.

For the induction step assume that $\operatorname{Hyp} 2_{\Lambda_{N}}\left(M_{2}\right)$ is true. Then Lemma 2.33 gives

$$
\left\|G_{\sqrt{2} \Lambda_{N}} f\right\|_{L^{2}\left(\mathbb{R}^{d}\right)} \leq\left\|\mathbb{1}_{\sqrt{2} \Lambda}\left(D_{v}\right) f_{0}\right\|_{L^{2}\left(\mathbb{R}^{d}\right)} e^{C_{f_{0}} T_{0}}=B
$$

and then, since $\epsilon(\alpha, 1) \leq \frac{2 m}{2 m+2}$ by our choice of $\alpha$, and $\Lambda_{N+1}=\widetilde{\Lambda}_{N}$, Lemma 2.34 shows that $\operatorname{Hyp} 2_{\Lambda_{N+1}}\left(M_{2}\right)$ is true, so by induction, it is true for all $N \in \mathbb{N}$. Invoking Lemma 2.33 again, we also have

$$
\left\|G_{\sqrt{2} \Lambda_{N}} f\right\|_{L^{2}\left(\mathbb{R}^{d}\right)} \leq B
$$

for all $N \in \mathbb{N}$ and letting $N \rightarrow \infty$, we see $\|G f\|_{L^{2}\left(\mathbb{R}^{d}\right)} \leq B$, which concludes the proof of Theorem 2.30 .

3. Removing the $L^{2}$ CONSTRAint: GeVRey REgUlarity AND (ULTRA-)ANALYTICITY OF WEAK SOLUTIONS

In this section we will give the proofs of Theorem 1.6, 1.8, and 1.9 in a slightly more general form. More precisely, we will prove

Theorem 3.1 (Gevrey smoothing I). Assume that the cross-section b satisfies the singularity condition (3) and the integrability condition (4) for $d \geq 2$, and for $d=1, b_{1}$ satisfies the singularity condition (6) and the integrability condition (7) for some $0<v<1$. Let $f$ be a weak solution of the Cauchy problem (1) with initial datum $f_{0} \geq 0$ and $f_{0} \in L_{m}^{1}\left(\mathbb{R}^{d}\right) \cap L \log L\left(\mathbb{R}^{d}\right)$ for some integer $m \geq 2$. Then, for all $0<\alpha \leq \min \left\{\alpha_{m, d}, v\right\}$,

$$
f(t, \cdot) \in G^{\frac{1}{2 \alpha}}\left(\mathbb{R}^{d}\right)
$$

for all $t>0$, where $\alpha_{m, d}=\frac{\log [(4 m+d) /(2 m+d)]}{\log 2}$. 
Theorem 3.2 (Gevrey smoothing II). Let $d \geq 2$. Assume that the cross-section $b$ satisfies the conditions of Theorem 1.6. Let $f$ be a weak solution of the Cauchy problem (1) with initial datum $f_{0} \geq 0$ and $f_{0} \in L_{m}^{1}\left(\mathbb{R}^{d}\right) \cap L \log L\left(\mathbb{R}^{d}\right)$ for some integer $m \geq 2$. Then, for all $0<\alpha \leq \min \left\{\alpha_{m, 2}, v\right\}$,

$$
f(t, \cdot) \in G^{\frac{1}{2 \alpha}}\left(\mathbb{R}^{d}\right)
$$

for all $t>0$, where $\alpha_{m, 2}=\frac{\log [(4 m+2) /(2 m+2)]}{\log 2}$. In particular, the weak solution is real analytic if $v=\frac{1}{2}$ and ultra-analytic if $v>\frac{1}{2}$ in any dimension.

If the integrability conditions (4) is replaced by the slightly stronger condition (16), which is true in all physically relevant cases, we can prove the stronger result

Theorem 3.3 (Gevrey smoothing III). Let $d \geq 2$. Assume that the cross-section $b$ satisfies the conditions of Theorem 1.6 and the condition (16), that is, they are bounded away from the singularity. Let $f$ be a weak solution of the Cauchy problem (1) with initial datum $f_{0} \geq 0$ and $f_{0} \in L_{m}^{1}\left(\mathbb{R}^{d}\right) \cap L \log L\left(\mathbb{R}^{d}\right)$ for some integer $m \geq 2$. Then, for all $0<\alpha \leq \min \left\{\alpha_{m, 1}, v\right\}$,

$$
f(t, \cdot) \in G^{\frac{1}{2 \alpha}}\left(\mathbb{R}^{d}\right)
$$

for all $t>0$, where $\alpha_{m, 1}=\frac{\log [(4 m+1) /(2 m+1)]}{\log 2}$.

\section{Remark 3.4.}

We even have the uniform bound

Corollary 3.5. Under the same assumptions as in Theorem 3.1 (or 3.2, respectively 3.3), for any weak solution $f$ of the Cauchy problem (1) initial datum $f_{0} \geq 0$ and $f_{0} \in L_{m}^{1}\left(\mathbb{R}^{d}\right) \cap L \log L\left(\mathbb{R}^{d}\right)$ for some integer $m \geq 2$ and for any $0<\alpha \leq \min \left\{\alpha_{d, m}, \nu\right\}$ (or any $0<\alpha \leq \min \left\{\alpha_{m, 2}, \nu\right\}$, respectively $\left.0<\alpha \leq \min \left\{\alpha_{m, 1}, v\right\}\right)$ there exist constants $0<K, C<\infty$ such that

$$
\sup _{0 \leq t<\infty} \sup _{\eta \in \mathbb{R}^{d}} e^{K \min (t, 1)\langle\eta\rangle^{2 \alpha}}|\hat{f}(t, \eta)| \leq C .
$$

Proof of Theorems 3.1 through 3.3. In the case where the initial condition $f_{0}$ obeys $f_{0} \geq 0$ and $f_{0} \in L_{m}^{1}\left(\mathbb{R}^{d}\right) \cap L \log L\left(\mathbb{R}^{d}\right)$ for some integer $m \geq 2$, but is not necessarily in $L^{2}\left(\mathbb{R}^{d}\right)$, we use the known $H^{\infty}$ smoothing of the Boltzmann $[16,4,30]$ and Kac equation ${ }^{4}[23]$ in a mild way (see also Appendix B): for $\tau>0$ one has $f(\tau, \cdot) \in L^{2}\left(\mathbb{R}^{d}\right)$ and using this as a new initial condition in Theorems 1.6 through 1.9 , and noting that $T_{0}$ in those theorems is arbitrary, this implies that $f(t, \cdot) \in G^{\frac{1}{2 \alpha}}\left(\mathbb{R}^{d}\right)$ for $t>0$.

Proof of Corollary 3.5. Using known results about propagation of Gevrey regularity by DesviLLETtes, Furioli, and Terraneo [15] for the non-cutoff homogeneous Boltzmann and Kac equation for Maxwellian molecules, the bounds from Corollary 2.21 through 2.37 extend to all times.

\section{ApPendix A. $L^{2}$ TYPe REFormulation of the Boltzmann AND KaC EQUATIONS}

A reformulation of the weak form (9) of the Boltzmann and Kac equations is derived. We want to choose a suitable test function $\varphi$ in terms of the weak solution $f$ itself in the weak formulation of the Cauchy problem (1). We use $\varphi(t, \cdot):=G_{\Lambda}^{2}\left(t, D_{v}\right) f(t, \cdot)$ and since this involves a hard cut-off in Fourier space, we automatically have high regularity of $\varphi(t, v)$ in the velocity variable, the question is to have $\mathscr{C}^{1}$ regularity in the time variable. For this we follow the strategy by Мовімото et al. [30].

\footnotetext{
${ }^{4} \mathrm{~A} H^{\infty}$ smoothing effect for the homogeneous non-cutoff Kac equation was first proved by L. DesvilletTes [11], but under the stronger assumption that all polynomial moments of the initial datum $f_{0}$ are bounded, i.e. $f_{0} \in L_{k}^{1}(\mathbb{R}) \cap L \log L(\mathbb{R})$ for all $k \in \mathbb{N}$.
} 
Proposition A.1. Let $f$ be a weak solution of the Cauchy problem (1) with initial datum $f_{0}$ satisfying (8), and let $T_{0}>0$. Then for all $t \in\left(0, T_{0}\right], \beta>0, \alpha \in(0,1)$, and $\Lambda>0$ we have $G_{\Lambda} f \in$ $\mathscr{C}\left(\left[0, T_{0}\right] ; L^{2}\left(\mathbb{R}^{d}\right)\right)$ and

$$
\begin{aligned}
& \frac{1}{2}\left\|G_{\Lambda}\left(t, D_{v}\right) f(t, \cdot)\right\|_{L^{2}\left(\mathbb{R}^{d}\right)}^{2}-\frac{1}{2} \int_{0}^{t}\left\langle f(\tau, \cdot),\left(\partial_{t} G_{\Lambda}^{2}\left(\tau, D_{v}\right)\right) f(\tau, \cdot)\right\rangle \mathrm{d} \tau \\
& =\frac{1}{2}\left\|\mathbb{1}_{\Lambda}\left(D_{v}\right) f_{0}\right\|_{L^{2}\left(\mathbb{R}^{d}\right)}^{2}+\int_{0}^{t}\left\langle Q(f, f)(\tau, \cdot), G_{\Lambda}^{2}\left(\tau, D_{v}\right) f(\tau, \cdot)\right\rangle \mathrm{d} \tau .
\end{aligned}
$$

To ensure that we can use $G_{\Lambda}^{2} f$ as a test function in the weak formulation of the Boltzmann equation, we need the following bilinear estimate on $Q(g, f)$, which is a special case of a larger class of functional inequalities by Alexandre [1, 2, 5].

Lemma A.2 (Functional Estimate on Collision Operator). Assume that the angular collision crosssection $b$ satisfies assumptions (3)-(4) or (6)-(7), respectively. Then for any $k>\frac{d+4}{2}$ there exists $a$ constant $C>0$ such that

$$
\|Q(g, f)\|_{H^{-k}\left(\mathbb{R}^{d}\right)} \leq C\|g\|_{L_{2}^{1}\left(\mathbb{R}^{d}\right)}\|f\|_{L_{2}^{1}\left(\mathbb{R}^{d}\right)} .
$$

Proof. This is a direct consequence ${ }^{5}$ of Theorem 7.4 in ALEXANDRE's review [2]: under the assumptions on $b$, for any $m \in \mathbb{R}$ there exists a constant $\widetilde{C}>0$ such that

$$
\|Q(g, f)\|_{H^{-m}\left(\mathbb{R}^{d}\right)} \leq \widetilde{C}\|g\|_{L_{2 v}^{1}\left(\mathbb{R}^{d}\right)}\|f\|_{H_{2 v}^{-m+2 v}\left(\mathbb{R}^{d}\right)} .
$$

Since $L^{1}\left(\mathbb{R}^{d}\right) \subset H^{-s}\left(\mathbb{R}^{d}\right)$ for any $s>\frac{d}{2}$, we obtain for $k>\frac{d+4}{2}$ and $v \in(0,1)$,

$$
\|f\|_{H_{2 v}^{-k+2 v}\left(\mathbb{R}^{d}\right)}=\left\|\langle\cdot\rangle^{2 v} f\right\|_{H^{-k+2 v}\left(\mathbb{R}^{d}\right)} \leq C\left\|\langle\cdot\rangle^{2 v} f\right\|_{L^{1}\left(\mathbb{R}^{d}\right)} \leq c\left\|\langle\cdot\rangle^{2} f\right\|_{L^{1}\left(\mathbb{R}^{d}\right)}=c\|f\|_{L_{2}^{1}\left(\mathbb{R}^{d}\right)} .
$$

i.e. $L_{2}^{1}\left(\mathbb{R}^{d}\right) \subset H_{2 v}^{-k+2 v}\left(\mathbb{R}^{d}\right)$ for any $k>\frac{d+4}{2}$ and $v \in(0,1)$. Therefore,

$$
\|Q(g, f)\|_{H^{-k}\left(\mathbb{R}^{d}\right)} \leq \widetilde{C}\|g\|_{L_{2 v}^{1}\left(\mathbb{R}^{d}\right)}\|f\|_{H_{2 v}^{-k+2 v}\left(\mathbb{R}^{d}\right)} \leq C\|g\|_{L_{2}^{1}\left(\mathbb{R}^{d}\right)}\|f\|_{L_{2}^{1}\left(\mathbb{R}^{d}\right)} .
$$

Lemma A. 2 implies that for $f, g \in L_{2}^{1}\left(\mathbb{R}^{d}\right),\langle Q(g, f), h\rangle$ is well-defined for all $h \in H^{k}\left(\mathbb{R}^{d}\right), k>\frac{d+4}{2}$, and one has $\langle Q(g, f), h\rangle=\langle\widehat{Q(g, f)}, \widehat{h}\rangle_{L^{2}}$.

Proof of Proposition A.1. Choosing a constant in time test function $\varphi(t, \cdot)=\psi \in \mathscr{C}_{0}^{\infty}\left(\mathbb{R}^{d}\right)$ in the weak formulation (9) yields

$$
\int_{\mathbb{R}^{d}} f(t, v) \psi(v) \mathrm{d} v-\int_{\mathbb{R}^{d}} f(s, v) \psi(v) \mathrm{d} v=\int_{s}^{t}\langle Q(f, f)(\tau, \cdot), \psi\rangle \mathrm{d} \tau, \quad \text { for } \quad 0 \leq s \leq t \leq T_{0}
$$

for all $\psi \in \mathscr{C}_{0}^{\infty}\left(\mathbb{R}^{d}\right)$ (this was already remarked by VILLANI [39] as an equivalent formulation of (9)). By means of (73) this equality can be extended to test functions $\psi \in H^{k}$ for $k>\frac{d+4}{2}$, in particular one can choose $\psi=G_{\Lambda}^{2} f(t, \cdot)$ and $\psi=G_{\Lambda}^{2} f(s, \cdot)$ which, taking the sum of both resulting equations, yields

$$
\begin{aligned}
& \left\|G_{\Lambda} f(t, \cdot)\right\|_{L^{2}\left(\mathbb{R}^{d}\right)}^{2}-\left\|G_{\Lambda} f(s, \cdot)\right\|_{L^{2}\left(\mathbb{R}^{d}\right)}^{2}=\left\langle f(t, \cdot), G_{\Lambda}^{2} f(t, \cdot)\right\rangle-\left\langle f(s, \cdot), G_{\Lambda}^{2} f(s, \cdot)\right\rangle \\
& =\left\langle f(t, \cdot),\left(G_{\Lambda}^{2}\left(t, D_{v}\right)-G_{\Lambda}^{2}\left(s, D_{v}\right)\right) f(s, \cdot)\right\rangle+\int_{s}^{t}\left\langle Q(f, f)(\tau, \cdot), G_{\Lambda}^{2} f(t, \cdot)+G_{\Lambda}^{2} f(s, \cdot)\right\rangle \mathrm{d} \tau .
\end{aligned}
$$

\footnotetext{
${ }^{5}$ This result is proved in [2] for $d=3$, but the proof depends only on assumption (3) and general properties of Littlewood-Paley decompositions and holds in any dimension $d \geq 1$.
} 
Using Plancherel, the first term on the right hand side of (74) can be estimated by

$$
\begin{aligned}
& \left|\left\langle f(t, \cdot),\left(G_{\Lambda}^{2}\left(t, D_{v}\right)-G_{\Lambda}^{2}\left(s, D_{v}\right)\right) f(s, \cdot)\right\rangle\right|=\left|\left\langle\hat{f}(t, \cdot),\left(G_{\Lambda}^{2}(t, \cdot)-G_{\Lambda}^{2}(s, \cdot)\right) \hat{f}(s, \cdot)\right\rangle\right| \\
& \quad \leq \int_{\mathbb{R}^{d}}|\hat{f}(t, \eta)|\left|G_{\Lambda}^{2}(t, \eta)-G_{\Lambda}^{2}(s, \eta)\right||\hat{f}(s, \eta)| \mathrm{d} \eta \\
& \quad \leq|t-s| \int_{\mathbb{R}^{d}} 2 \beta\langle\eta\rangle^{2 \alpha} G_{\Lambda}^{2}(t, \eta) \mathrm{d} \eta\|f(t, \cdot)\|_{L^{1}\left(\mathbb{R}^{d}\right)}\|f(s, \cdot)\|_{L^{1}\left(\mathbb{R}^{d}\right)} \leq C_{\Lambda, T_{0}}|t-s|\left\|f_{0}\right\|_{L^{1}\left(\mathbb{R}^{d}\right)}^{2},
\end{aligned}
$$

and, using that the terms involving the collision operator can, for any $k>\frac{d+4}{2}$ (compare (73)), be bounded by

$$
\begin{aligned}
\left|\left\langle Q(f, f)(\tau, \cdot), G_{\Lambda}^{2} f(t, \cdot)\right\rangle\right| & \leq\|Q(f, f)(\tau, \cdot)\|_{H^{-k}\left(\mathbb{R}^{d}\right)}\left\|G_{\Lambda}^{2} f(t, \cdot)\right\|_{H^{k}\left(\mathbb{R}^{d}\right)} \\
& \leq C\|f\|_{L_{2}^{1}\left(\mathbb{R}^{d}\right)}^{2}\left(\int_{\mathbb{R}^{d}}\langle\eta\rangle^{2 k} G_{\Lambda}^{4}(t, \eta)|\hat{f}(t, \eta)|^{2} \mathrm{~d} \eta\right)^{1 / 2} \\
& \leq C\|f\|_{L_{2}^{1}\left(\mathbb{R}^{d}\right)}^{2}\|f(t, \cdot)\|_{L^{1}\left(\mathbb{R}^{d}\right)}\left(\int_{\mathbb{R}^{d}}\langle\eta\rangle^{2 k} G_{\Lambda}^{4}\left(T_{0}, \eta\right) \mathrm{d} \eta\right)^{1 / 2} \\
& \leq C_{\Lambda, T_{0}}^{\prime}\left\|f_{0}\right\|_{L_{2}^{1}\left(\mathbb{R}^{d}\right)}^{2}\left\|f_{0}\right\|_{L^{1}\left(\mathbb{R}^{d}\right)}
\end{aligned}
$$

for any $t \in\left[0, T_{0}\right]$, yields

$$
\left|\int_{s}^{t}\left\langle Q(f, f)(\tau, \cdot), G_{\Lambda}^{2} f(t, \cdot)+G_{\Lambda}^{2} f(s, \cdot)\right\rangle \mathrm{d} \tau\right| \leq 2 C_{\Lambda, T_{0}}^{\prime}|t-s|\left\|f_{0}\right\|_{L_{2}^{1}\left(\mathbb{R}^{d}\right)}^{2}\left\|f_{0}\right\|_{L^{1}\left(\mathbb{R}^{d}\right)} .
$$

Plugging the latter two bounds into (74) shows that $G_{\Lambda} f \in \mathscr{C}\left(\left[0, T_{0}\right] ; L^{2}\left(\mathbb{R}^{d}\right)\right)$, in fact, the map $\left[0, T_{0}\right] \ni t \mapsto\left\|G_{\Lambda} f(t, \cdot)\right\|_{L^{2}\left(\mathbb{R}^{d}\right)}$ is even Lipschitz continuous.

For any test function $\varphi \in \mathscr{C}^{1}\left(\mathbb{R}^{+} ; \mathscr{C}_{0}^{\infty}\left(\mathbb{R}^{d}\right)\right)$ the term involving the partial derivative $\partial_{t} \varphi$ in the weak formulation (9) can be rewritten as

$$
\int_{0}^{t}\left\langle f(\tau, \cdot), \partial_{\tau} \varphi(\tau, \cdot)\right\rangle \mathrm{d} \tau=\lim _{h \rightarrow 0} \int_{0}^{t}\left\langle f(\tau, \cdot)+f(\tau+h, \cdot), \frac{\varphi(\tau+h, \cdot)-\varphi(\tau, \cdot)}{2 h}\right\rangle \mathrm{d} \tau
$$

since $f \in \mathscr{C}\left(\mathbb{R}^{+} ; \mathscr{D}^{\prime}\left(\mathbb{R}^{d}\right)\right)$. The integral on the right hand side is well-defined even for $\varphi \in$ $L^{\infty}\left(\left[0, T_{0}\right] ; W^{2, \infty}\left(\mathbb{R}^{d}\right)\right)$, in particular for $\varphi=G_{\Lambda}^{2} f$, yielding

$$
\begin{aligned}
& \int_{0}^{t}\left\langle f(\tau, \cdot)+f(\tau+h, \cdot), \frac{\varphi(\tau+h, \cdot)-\varphi(\tau, \cdot)}{2 h}\right\rangle \mathrm{d} \tau \\
& =\int_{0}^{t}\left\langle f(\tau, \cdot)+f(\tau+h, \cdot), \frac{G_{\Lambda}^{2} f(\tau+h, \cdot)-G_{\Lambda}^{2} f(\tau, \cdot)}{2 h}\right\rangle \mathrm{d} \tau \\
& =\frac{1}{2 h} \int_{0}^{t}\left(\left\|G_{\Lambda} f(\tau+h, \cdot)\right\|_{L^{2}}^{2}-\left\|G_{\Lambda} f(\tau, \cdot)\right\|_{L^{2}}^{2}\right) \mathrm{d} \tau \\
& \quad+\int_{0}^{t}\left\langle f(\tau, \cdot), \frac{G_{\Lambda}^{2}\left(\tau+h, D_{v}\right)-G_{\Lambda}^{2}\left(\tau, D_{v}\right)}{2 h} f(\tau+h, \cdot)\right\rangle \mathrm{d} \tau .
\end{aligned}
$$

Using $G_{\Lambda} f \in \mathscr{C}\left(\left[0, T_{0}\right] ; L^{2}\left(\mathbb{R}^{d}\right)\right)$ it follows that

$$
\begin{aligned}
& \frac{1}{2 h} \int_{0}^{t}\left(\left\|G_{\Lambda} f(\tau+h, \cdot)\right\|_{L^{2}\left(\mathbb{R}^{d}\right)}^{2}-\left\|G_{\Lambda} f(\tau, \cdot)\right\|_{L^{2}\left(\mathbb{R}^{d}\right)}^{2}\right) \mathrm{d} \tau \\
& =\frac{1}{2 h} \int_{t}^{t+h}\left\|G_{\Lambda} f(\tau, \cdot)\right\|_{L^{2}\left(\mathbb{R}^{d}\right)}^{2} \mathrm{~d} \tau-\frac{1}{2 h} \int_{0}^{h}\left\|G_{\Lambda} f(\tau, \cdot)\right\|_{L^{2}\left(\mathbb{R}^{d}\right)}^{2} \mathrm{~d} \tau \\
& \stackrel{h \rightarrow 0}{\longrightarrow} \frac{1}{2}\left\|G_{\Lambda} f(t, \cdot)\right\|_{L^{2}\left(\mathbb{R}^{d}\right)}^{2}-\frac{1}{2}\left\|G_{\Lambda} f(0, \cdot)\right\|_{L^{2}\left(\mathbb{R}^{d}\right)}^{2} \cdot
\end{aligned}
$$


where $\left\|G_{\Lambda} f(0, \cdot)\right\|_{L^{2}\left(\mathbb{R}^{d}\right)}=\left\|\mathbb{1}_{\Lambda}\left(D_{v}\right) f_{0}\right\|_{L^{2}\left(\mathbb{R}^{d}\right)}$. For the second integral, an application of dominated convergence gives

$$
\begin{aligned}
& \lim _{h \rightarrow 0} \int_{0}^{t}\left\langle f(\tau, \cdot), \frac{G_{\Lambda}^{2}\left(\tau+h, D_{v}\right)-G_{\Lambda}^{2}\left(\tau, D_{v}\right)}{2 h} f(\tau+h, \cdot)\right\rangle \mathrm{d} \tau \\
& \quad=\frac{1}{2} \int_{0}^{t}\left\langle f(\tau, \cdot),\left(\partial_{\tau} G_{\Lambda}^{2}\right)\left(\tau, D_{v}\right) f(\tau, \cdot)\right\rangle \mathrm{d} \tau .
\end{aligned}
$$

Putting everything together, we thus have proved equation (72), i.e.

$$
\begin{aligned}
\frac{1}{2}\left\|G_{\Lambda} f\right\|_{L^{2}\left(\mathbb{R}^{d}\right)}^{2}=\frac{1}{2}\left\|\mathbb{1}_{\Lambda}\left(D_{v}\right) f_{0}\right\|_{L^{2}\left(\mathbb{R}^{d}\right)}^{2} & +\frac{1}{2} \int_{0}^{t}\left\langle f(\tau, \cdot),\left(\partial_{\tau} G_{\Lambda}^{2}\right)\left(\tau, D_{v}\right) f(\tau, \cdot)\right\rangle \mathrm{d} \tau \\
& +\int_{0}^{t}\left\langle Q(f, f), G_{\Lambda}^{2} f\right\rangle \mathrm{d} \tau .
\end{aligned}
$$

Appendix B. $H^{\infty}$ SMOOThing of the Boltzmann an Kac EQUations

We follow the strategy as in our proof of Gevrey regularity, with several simplifications. Of course, we do not assume that $f_{0}$ is square integrable! We have

Theorem B.1 ( $H^{\infty}$ smoothing for the homogeneous Boltzmann and Kac equation). Assume that the cross-section $b$ satisfies (3)-(4) for $d \geq 2$, respectively (6)-(7) for $d=1$, with $0<v<1$. Let $f$ be a weak solution of the Cauchy problem (1) with initial datum satisfying conditions (8). Then

$$
f(t, \cdot) \in H^{\infty}\left(\mathbb{R}^{d}\right)
$$

for all $t>0$.

The proof is known, at least for the three dimensional Boltzmann equation see [30], we give a proof for the convenience of the reader. Again, one has to use suitable time-dependent Fourier multipliers. Note that for $f_{0} \in L^{1}\left(\mathbb{R}^{d}\right)$ one has

$$
\left\|f_{0}\right\|_{H^{-\gamma}\left(\mathbb{R}^{d}\right)} \leq C_{d, \gamma}\left\|f_{0}\right\|_{L^{1}\left(\mathbb{R}^{d}\right)}
$$

with $C_{d, \gamma}=\left(\int_{\mathbb{R}^{d}}\langle\eta\rangle^{-\gamma} \mathrm{d} \eta\right)^{1 / 2}$ which is finite for all $\gamma>d / 2$. We choose $\gamma=d$, for convenience, and

as a multiplier. Then

$$
M_{\Lambda}(t, \eta):=\langle\eta\rangle^{-d} e^{\beta t \log \langle\eta\rangle} \mathbb{1}_{\Lambda}(|\eta|)
$$

$$
\sup _{\Lambda>0}\left\|M_{\Lambda}\left(0, D_{v}\right) f_{0}\right\|_{L^{2}\left(\mathbb{R}^{d}\right)}=\left\|M_{\infty}(0, \cdot) \hat{f_{0}}\right\|_{L^{2}\left(\mathbb{R}^{d}\right)}=\left\|f_{0}\right\|_{H^{-d}\left(\mathbb{R}^{d}\right)} \leq C_{d, d}\left\|f_{0}\right\|_{L^{1}\left(\mathbb{R}^{d}\right)}
$$

The proof of Proposition A.1 carries over and we have

$$
\begin{aligned}
& \frac{1}{2}\left\|M_{\Lambda}\left(t, D_{v}\right) f(t, \cdot)\right\|_{L^{2}\left(\mathbb{R}^{d}\right)}^{2}-\frac{1}{2} \int_{0}^{t}\left\langle f(\tau, \cdot),\left(\partial_{\tau} M_{\Lambda}^{2}\left(\tau, D_{v}\right)\right) f(\tau, \cdot)\right\rangle \mathrm{d} \tau \\
& =\frac{1}{2}\left\|M_{\Lambda}\left(0, D_{v}\right) f_{0}\right\|_{L^{2}\left(\mathbb{R}^{d}\right)}^{2}+\int_{0}^{t}\left\langle Q(f, f)(\tau, \cdot), M_{\Lambda}^{2}\left(\tau, D_{v}\right) f(\tau, \cdot)\right\rangle \mathrm{d} \tau .
\end{aligned}
$$

and as in the proof of Corollary 2.4, we have

$$
\begin{aligned}
\left\langle Q(f, f), M_{\Lambda}^{2} f\right\rangle & =\left\langle Q\left(f, M_{\Lambda} f\right), M_{\Lambda} f\right\rangle+\left\langle M_{\Lambda} Q(f, f)-Q\left(f, M_{\Lambda} f\right), M_{\Lambda} f\right\rangle \\
& \leq-\widetilde{C}_{f_{0}}\left\|M_{\Lambda} f\right\|_{H^{v}}^{2}+C_{f_{0}}\left\|M_{\Lambda} f\right\|_{L^{2}}^{2}+\left\langle M_{\Lambda} Q(f, f)-Q\left(f, M_{\Lambda} f\right), M_{\Lambda} f\right\rangle
\end{aligned}
$$

The replacement of Proposition 2.9 is

Proposition B.2. The commutation error is bounded by

$$
\left|\left\langle M_{\Lambda} Q(f, f)-Q\left(f, M_{\Lambda} f\right), M_{\Lambda} f\right\rangle\right| \leq\left(1+2^{d-1}\right) c_{b, d}\|f\|_{L^{1}}\left(\frac{d}{2}+\frac{\beta t}{2} 2^{\beta t / 2}\right)\left\|M_{\Lambda} f\right\|_{L^{2}}^{2}
$$

with the constant $c_{b, d}$ from Lemma 2.25 . 
Remark B.3. Of course, for any weak solution $f$ of the Boltzmann and Kac equations, $\|f\|_{L^{1}}=$ $\|f(t, \cdot)\|_{L^{1}}=\left\|f_{0}\right\|_{L^{1}}$. The fact that the commutator is bounded in terms of the $L^{2}$ norm of $M_{\Lambda} f$ makes the proof of $H^{\infty}$ smoothing for the Boltzmann and Kac equations much simpler than the proof of Gevrey regularity.

Proof. As in the proof of Proposition 2.9, Bobylev's formula shows

$$
\begin{aligned}
& \left|\left\langle M_{\Lambda} Q(f, f)-Q\left(f, M_{\Lambda} f\right), M_{\Lambda} f\right\rangle\right| \leq \\
& \quad \leq \int_{\mathbb{R}^{d}} \int_{\mathbb{S}^{d-1}} b\left(\frac{\eta}{|\eta|} \cdot \sigma\right) M_{\Lambda}(\eta)\left|\hat{f}(\eta)\left\|\hat{f}\left(\eta^{-}\right)\right\| \hat{f}\left(\eta^{+}\right) \| M_{\Lambda}(t, \eta)-M_{\Lambda}\left(t, \eta^{+}\right)\right| \mathrm{d} \sigma \mathrm{d} \eta \\
& \quad \leq\|\hat{f}\|_{L^{\infty}} \int_{\mathbb{R}^{d}} \int_{\mathbb{S}^{d-1}} b\left(\frac{\eta}{|\eta|} \cdot \sigma\right) M_{\Lambda}(\eta)\left|\hat{f}(\eta)\left\|\hat{f}\left(\eta^{+}\right)\right\| M_{\Lambda}(t, \eta)-M_{\Lambda}\left(t, \eta^{+}\right)\right| \mathrm{d} \sigma \mathrm{d} \eta
\end{aligned}
$$

where, as before, $\eta^{ \pm}=\frac{1}{2}(\eta \pm|\eta| \sigma)$. To bound $\left|M_{\Lambda}(\eta)-M_{\Lambda}\left(\eta^{+}\right)\right|$, we let $s:=|\eta|^{2}$ and $s^{+}=\left|\eta^{+}\right|^{2}$. Recall that $\left|\eta^{+}\right|^{2}=\frac{|\eta|^{2}}{2}\left(1+\frac{\eta}{|\eta|} \cdot \sigma\right)$ and

$$
1-\frac{s^{+}}{s}=1-\frac{\left|\eta^{+}\right|^{2}}{|\eta|^{2}}=\frac{1}{2}\left(1-\frac{\eta}{|\eta|} \cdot \sigma\right)
$$

Again, because of the support condition on the collision kernel $b(\cos \theta)$, we have $\frac{s}{2} \leq s^{+} \leq s$. Set $\widetilde{M}(s):=(1+s)^{-d / 2} e^{\frac{\beta t}{2} \log (1+s)}$. Then, for $|\eta| \leq \Lambda$,

$$
\begin{aligned}
M_{\Lambda}(\eta) & -M_{\Lambda}\left(\eta^{+}\right)=\widetilde{M}(s)-\widetilde{M}\left(s^{+}\right)=(1+s)^{-d / 2} e^{\frac{\beta t}{2} \log (1+s)}-\left(1+s^{+}\right)^{-d / 2} e^{\frac{\beta t}{2} \log \left(1+s^{+}\right)} \\
& =(1+s)^{-d / 2}\left(e^{\frac{\beta t}{2} \log (1+s)}-e^{\frac{\beta t}{2} \log \left(1+s^{+}\right)}\right)+\left((1+s)^{-d / 2}-\left(1+s^{+}\right)^{-d / 2}\right) e^{\frac{\beta t}{2} \log \left(1+s^{+}\right)} .
\end{aligned}
$$

Since $s \leq 2 s^{+}$, we have $\left(1+s^{+}\right)^{-1} \leq 2(1+s)^{-1}$. Hence

$$
\begin{aligned}
\left|(1+s)^{-d / 2}-\left(1+s^{+}\right)^{-d / 2}\right| & =\frac{d}{2} \int_{s^{+}}^{s}(1+r)^{-d / 2-1} \mathrm{~d} r \leq \frac{d}{2}\left(1+s^{+}\right)^{-d / 2-1}\left(s-s^{+}\right) \\
& \leq d\left(1+s^{+}\right)^{-d / 2}\left(1-\frac{s^{+}}{s}\right)
\end{aligned}
$$

In addition, $\log (1+s) \leq \log \left(2\left(1+s^{+}\right)\right)=\log 2+\log \left(1+s^{+}\right)$. So

$$
\begin{aligned}
\left|e^{\frac{\beta t}{2} \log (1+s)}-e^{\frac{\beta t}{2} \log \left(1+s^{+}\right)}\right| & \leq \frac{\beta t}{2} \int_{s^{+}}^{s} \frac{1}{1+r} e^{\frac{\beta t}{2} \log (1+r)} \mathrm{d} r \leq \frac{\beta t}{2} \frac{s}{1+s^{+}} e^{\frac{\beta t}{2} \log (1+s)}\left(1-\frac{s^{+}}{s}\right) \\
& \leq \beta t 2^{\frac{\beta t}{2}} e^{\frac{\beta t}{2} \log \left(1+s^{+}\right)}\left(1-\frac{s^{+}}{s}\right) .
\end{aligned}
$$

Also $\log (1+s) \leq \log \left(2\left(1+s^{+}\right)\right)=\log 2+\log \left(1+s^{+}\right)$. These bounds together with (80) show

$$
\left|M_{\Lambda}(\eta)-M_{\Lambda}\left(\eta^{+}\right)\right| \leq\left(d+\beta t 2^{\frac{\beta t}{2}}\right)\left(1-\frac{\left|\eta^{+}\right|^{2}}{|\eta|^{2}}\right) M_{\Lambda}\left(\eta^{+}\right)
$$

for all $|\eta| \leq \Lambda$. Since the integration in (79) is only over $|\eta| \leq \Lambda$, plugging this together with $\|\hat{f}\|_{L^{\infty}} \leq\|f\|_{L^{1}}$ into (79) yields

$$
\begin{aligned}
& \left|\left\langle M_{\Lambda} Q(f, f)-Q\left(f, M_{\Lambda} f\right), M_{\Lambda} f\right\rangle\right| \\
& \quad \leq\|f\|_{L^{1}}\left(d+\beta t 2^{\frac{\beta t}{2}}\right) \int_{\mathbb{R}^{d}} \int_{\mathbb{S}^{d-1}} b\left(\frac{\eta}{|\eta|} \cdot \sigma\right)\left(1-\frac{\left|\eta^{+}\right|^{2}}{|\eta|^{2}}\right) M_{\Lambda}(\eta)|\hat{f}(\eta)| M_{\Lambda}\left(\eta^{+}\right)\left|\hat{f}\left(\eta^{+}\right)\right| \mathrm{d} \sigma \mathrm{d} \eta .
\end{aligned}
$$

Noting again

$$
M_{\Lambda}(\eta)|\hat{f}(\eta)| M_{\Lambda}\left(\eta^{+}\right)\left|\hat{f}\left(\eta^{+}\right)\right| \leq \frac{1}{2}\left(\left(M_{\Lambda}(\eta)|\hat{f}(\eta)|\right)^{2}+\left(M_{\Lambda}\left(\eta^{+}\right)\left|\hat{f}\left(\eta^{+}\right)\right|\right)^{2}\right)
$$

and performing the same change of variables for the integral containing $\eta^{+}$as in the proof of Lemma 2.11 finishes the proof of equation (78).

Now we can finish the 
Proof of Theorem B.1. Using (76), (77), Proposition B.2, and

$$
\partial_{\tau} M_{\Lambda}(\tau, \eta)^{2}=2 \beta \log \langle\eta\rangle M_{\Lambda}(\tau, \eta)^{2}
$$

one sees

$$
\begin{aligned}
\left\|M_{\Lambda}\left(t, D_{v}\right) f(t, \cdot)\right\|_{L^{2}}^{2} \leq & \left\|f_{0}\right\|_{H^{-d}}^{2}+2 C_{f_{0}} \int_{0}^{t}\left\|M_{\Lambda}\left(\tau, D_{v}\right) f(\tau, \cdot)\right\|_{L^{2}}^{2} \mathrm{~d} \tau \\
& +\int_{0}^{t}\left\langle M_{\Lambda}\left(\tau, D_{v}\right) f(\tau, \cdot),\left(\beta \log \left\langle D_{v}\right\rangle-2 \widetilde{C}_{f_{0}}\left\langle D_{v}\right\rangle^{2 v}\right) M_{\Lambda}\left(\tau, D_{v}\right) f(\tau, \cdot)\right\rangle \mathrm{d} \tau \\
& +\left(1+2^{d-1}\right) c_{b, d}\left\|f_{0}\right\|_{L^{1}} \int_{0}^{t}\left(\frac{d}{2}+\frac{\beta \tau}{2} 2^{\frac{\beta \tau}{2}}\right)\left\|M_{\Lambda}\left(\tau, D_{v}\right) f(\tau, \cdot)\right\|_{L^{2}}^{2}
\end{aligned}
$$

Setting

$$
\begin{aligned}
A(\beta, \tau) & :=\sup _{\eta \in \mathbb{R}^{d}}\left(\beta \log \langle\eta\rangle-2 \widetilde{C}_{f_{0}}\langle\eta\rangle^{2 v}\right)+2 C_{f_{0}}+\left(1+2^{d-1}\right) c_{b, d}\left\|f_{0}\right\|_{L^{1}}\left(\frac{d}{2}+\frac{\beta \tau}{2} 2^{\frac{\beta \tau}{2}}\right) \\
& =\frac{\beta}{2 v}\left[\log \left(\frac{\beta}{4 v \widetilde{C}_{f_{0}}}\right)-1\right]+2 C_{f_{0}}+\left(1+2^{d-1}\right) c_{b, d}\left\|f_{0}\right\|_{L^{1}}\left(\frac{d}{2}+\frac{\beta \tau}{2} 2^{\frac{\beta \tau}{2}}\right)
\end{aligned}
$$

the above can be bounded by

$$
\left\|M_{\Lambda}\left(t, D_{v}\right) f(t, \cdot)\right\|_{L^{2}}^{2} \leq\left\|f_{0}\right\|_{H^{-d}}^{2}+\int_{0}^{t} A(\beta, \tau)\left\|M_{\Lambda}\left(\tau, D_{v}\right) f(\tau, \cdot)\right\|_{L^{2}}^{2} \mathrm{~d} \tau
$$

and from Gronwall's lemma we get

$$
\left\|M_{\Lambda}\left(t, D_{v}\right) f(t, \cdot)\right\|_{L^{2}}^{2} \leq\left\|f_{0}\right\|_{H^{-d}}^{2} \exp \left(\int_{0}^{t} A(\beta, \tau) \mathrm{d} \tau\right) .
$$

Letting $\Lambda \rightarrow \infty$ one sees

$$
\|f(t, \cdot)\|_{H^{\beta t-d}}^{2}=\left\|M_{\infty}\left(t, D_{v}\right) f(t, \cdot)\right\|_{L^{2}}^{2} \leq\left\|f_{0}\right\|_{H^{-d}}^{2} \exp \left(\int_{0}^{t} A(\beta, \tau) \mathrm{d} \tau\right) .
$$

that is, $f(t, \cdot) \in H^{\beta t-d}\left(\mathbb{R}^{d}\right)$. Now let $\beta \rightarrow \infty$ to see that $f(t, \cdot) \in H^{\infty}\left(\mathbb{R}^{d}\right)$ for any $t>0$.

Remark B.4. Setting $\beta=\frac{\gamma+d}{t}$, one sees that $\|f(t, \cdot)\|_{H^{\gamma}\left(\mathbb{R}^{d}\right)} \lesssim t^{-\frac{\gamma+d}{4 v}}$, so the $H^{\gamma}$ norms, in particular the $L^{2}$ norm, of $f(t, \cdot)$ blow up at most polynomially as $t \rightarrow 0$.

\section{Appendix C. The Kolmogorov-Landau inequality}

In this section we give a short proof of

Lemma C.1 (Kolmogorov-Landau inequality on the unit interval). Let $m \geq 2$ be an integer. There exists a constant $C_{m}>0$ such that for all $w \in W^{m, \infty}([0,1])$,

$$
\left\|w^{(k)}\right\|_{L^{\infty}([0,1])} \leq C_{m}\left(\frac{\|w\|_{L^{\infty}([0,1])}}{u^{k}}+u^{m-k}\left\|w^{(m)}\right\|_{L^{\infty}([0,1])}\right), \quad k=1, \ldots, m-1,
$$

for all $0<u \leq 1$.

For the convenience of the reader, we give a short proof. The following argument is in part borrowed from R. A. DeVore and G. G. Lorentz's book [17] (pp.37-39).

Proof. Since $w \in W^{m, \infty}([0,1])$, it has absolutely continuous derivatives of order up to $m-1$ and essentially bounded $m^{\text {th }}$ derivative.

Let $x \in\left[0, \frac{1}{2}\right]$ and $h \in\left[0, \frac{1}{2}\right]$. Then, by Taylor's theorem,

$$
w(x+h)=w(x)+\sum_{j=1}^{m-1} \frac{h^{j}}{j !} w^{(j)}(x)+R_{m}(x, h)
$$


with remainder $R_{m}(x, h)=\int_{0}^{h} \frac{(h-t)^{m-1}}{(m-1) !} w^{(m)}(x+t) \mathrm{d} t$, which can be bounded by

$$
\left|R_{m}(x, h)\right| \leq\left\|w^{(m)}\right\|_{L^{\infty}([0,1])} \int_{0}^{h} \frac{(h-t)^{m-1}}{(m-1) !} \mathrm{d} t=\frac{h^{m}}{m !}\left\|w^{(m)}\right\|_{L^{\infty}([0,1])} .
$$

Choosing $m-1$ real numbers $0<\lambda_{1}<\lambda_{2}<\cdots<\lambda_{m-1} \leq 1$ we obtain for $h \in\left[0, \frac{1}{2}\right]$ the system of equations

$$
\sum_{j=1}^{m-1} \lambda_{s}^{j} \frac{h^{j}}{j !} w^{(j)}(x)=w\left(x+\lambda_{s} h\right)-w(x)-R_{m}\left(x, \lambda_{s} h\right) \quad \text { for } s=1, \cdots, m-1 .
$$

Setting

$$
\begin{aligned}
V & =\left(\begin{array}{cccc}
\lambda_{1} & \lambda_{1}^{2} & \cdots & \lambda_{1}^{m-1} \\
\lambda_{2} & \lambda_{2}^{2} & \cdots & \lambda_{2}^{m-1} \\
\vdots & & \ddots & \vdots \\
\lambda_{m-1} & \lambda_{m-1}^{2} & \cdots & \lambda_{m-1}^{m-1}
\end{array}\right), \quad \mathbf{w}(x)=\left(\begin{array}{c}
h w^{\prime}(x) \\
\frac{h^{2}}{2} w^{\prime \prime}(x) \\
\vdots \\
\frac{h^{m-1}}{(m-1) !} w^{(m-1)}(x)
\end{array}\right), \\
\mathbf{b}(x) & =\left(\begin{array}{c}
w\left(x+\lambda_{1} h\right)-w(x)-R_{m}\left(x, \lambda_{1} h\right) \\
w\left(x+\lambda_{2} h\right)-w(x)-R_{m}\left(x, \lambda_{2} h\right) \\
\vdots \\
w\left(x+\lambda_{m-1} h\right)-w(x)-R_{m}\left(x, \lambda_{m-1} h\right)
\end{array}\right),
\end{aligned}
$$

we have $V \mathbf{w}(x)=\mathbf{b}(x)$. Since the Vandermonde determinant

$$
\operatorname{det} V=\prod_{i=1}^{m-1} \lambda_{i} \prod_{1 \leq j<l \leq m-1}\left(\lambda_{l}-\lambda_{j}\right) \neq 0
$$

$V$ is invertible and we obtain $\mathbf{w}(x)=V^{-1} \mathbf{b}(x)$ and therefore

$$
\left|\frac{h^{k}}{k !} w^{(k)}(x)\right| \leq\|\mathbf{w}(x)\| \leq\left\|V^{-1}\right\|\|\mathbf{b}(x)\| .
$$

where $\|\cdot\|$ is any norm on $\mathbb{R}^{m-1}$, respectively the induced operator norm on the space of $(m-1) \times$ $(m-1)$ real matrices. Choosing for concreteness the $\ell^{1}$ norm on $\mathbb{R}^{m-1}$, we have

$$
\|\mathbf{b}(x)\|=\sum_{s=1}^{m-1}\left|w\left(x+\lambda_{s} h\right)-w(x)-R_{m}\left(x, \lambda_{s} h\right)\right| \leq(m-1)\left(2\|w\|_{L^{\infty}([0,1])}+\frac{h^{m}}{m !}\left\|w^{(m)}\right\|_{L^{\infty}([0,1])}\right) .
$$

While for our application the size of $\left\|V^{-1}\right\|$ is of no importance, one can even explicitly calculate it: The inverse of the Vandermonde matrix $V$ is explicitly known (see for instance [18]),

$$
\left(V^{-1}\right)_{\alpha \beta}=(-1)^{\alpha-1} \frac{\sigma_{m-1-\alpha}^{\beta}}{\lambda_{\beta} \prod_{v \neq \beta}\left(\lambda_{v}-\lambda_{\beta}\right)}, \quad \alpha, \beta=1, \ldots, m-1,
$$

where $\sigma_{i}^{j}, i, j=1, \ldots, m-2$ is the $i^{\text {th }}$ elementary symmetric function in the $(m-2)$ variables $\lambda_{1}, \ldots, \lambda_{j-1}, \lambda_{j+1}, \ldots, \lambda_{m-1}$,

$$
\sigma_{i}^{j}=\sum_{\substack{1 \leq v_{1}<\cdots<v_{i} \leq m-1 \\ v_{1}, \ldots, v_{i} \neq j}} \lambda_{v_{1}} \cdots \lambda_{v_{i}}, \quad \sigma_{0}^{j}:=1
$$

By means of the identity (Lemma 1 in [18])

$$
\sum_{i=0}^{m-2} \sigma_{i}^{j}=\prod_{\substack{v=1 \\ v \neq j}}^{m-1}\left(1+\lambda_{v}\right)
$$


which holds since the $\lambda_{v}$ are all positive, we get

$$
\begin{aligned}
\left\|V^{-1}\right\| & =\max _{1 \leq \beta \leq m-1} \sum_{\alpha=1}^{m-1}\left|\left(V^{-1}\right)_{\alpha \beta}\right|=\max _{1 \leq \beta \leq m-1} \frac{1}{\lambda_{\beta} \prod_{\nu \neq \beta}\left|\lambda_{v}-\lambda_{\beta}\right|} \sum_{\alpha=1}^{m-1} \sigma_{m-1-\alpha}^{\beta} \\
& =\max _{1 \leq \beta \leq m-1} \frac{1}{\lambda_{\beta}} \prod_{\substack{\nu=1 \\
\nu \neq \beta}}^{m-1} \frac{1+\lambda_{v}}{\left|\lambda_{v}-\lambda_{\beta}\right|} .
\end{aligned}
$$

Going back to inequality (82), we have so far proved that

$$
\frac{h^{k}}{k !}\left|w^{(k)}(x)\right| \leq(m-1)\left\|V^{-1}\right\|\left(2\|w\|_{L^{\infty}([0,1])}+\frac{h^{m}}{m !}\left\|w^{(m)}\right\|_{L^{\infty}([0,1])}\right),
$$

which yields

$$
\begin{aligned}
\left|w^{(k)}(x)\right| & \leq(m-1)\left\|V^{-1}\right\|\left(\frac{2 k !}{h^{k}}\|w\|_{L^{\infty}([0,1])}+h^{m-k} \frac{k !}{m !}\left\|w^{(m)}\right\|_{L^{\infty}([0,1])}\right) \\
& \leq(m-1)\left\|V^{-1}\right\|\left(\frac{2 m !}{h^{k}}\|w\|_{L^{\infty}([0,1])}+h^{m-k}\left\|w^{(m)}\right\|_{L^{\infty}([0,1])}\right)
\end{aligned}
$$

For $x \in\left[\frac{1}{2}, 1\right]$ the same calculations with $h$ replaced by $-h$ prove inequality (83) also in this case, so

$$
\left\|w^{(k)}\right\|_{L^{\infty}([0,1])} \leq(m-1)\left\|V^{-1}\right\|\left(\frac{2 m !}{h^{k}}\|w\|_{L^{\infty}([0,1])}+h^{m-k}\left\|w^{(m)}\right\|_{L^{\infty}([0,1])}\right)
$$

for all $h \in\left[0, \frac{1}{2}\right]$. Taking an arbitrary $u \in[0,1]$, inequality (84) implies with $h=\frac{u}{2} \in\left[0, \frac{1}{2}\right]$,

$$
\left\|w^{(k)}\right\|_{L^{\infty}([0,1])} \leq 2^{m} m !(m-1)\left\|V^{-1}\right\|\left(\frac{1}{u^{k}}\|w\|_{L^{\infty}([0,1])}+u^{m-k}\left\|w^{(m)}\right\|_{L^{\infty}([0,1])}\right),
$$

which is the claimed inequality with

$$
C_{m}=2^{m} m !(m-1)\left\|V^{-1}\right\|=2^{m} m !(m-1) \max _{1 \leq \beta \leq m-1} \frac{1}{\lambda_{\beta}} \prod_{\substack{v=1 \\ v \neq \beta}}^{m-1} \frac{1+\lambda_{v}}{\left|\lambda_{v}-\lambda_{\beta}\right|} .
$$

Remark C.2. The constant $C_{m}$ in equality (85) is far from optimal, but can be made small by minimising in the choice of the points $0<\lambda_{1}<\cdots<\lambda_{m-1} \leq 1$, suggesting that the optimal constant might be obtained by methods from approximation theory.

Indeed, by a more refined argument making use of numerical differentiation formulas, the minimisers of the associated multiplicative Kolmogorov-Landau inequality, i.e., extremisers of

$$
M_{k}(\sigma):=\sup \left\{\left\|w^{(k)}\right\|_{L^{\infty}([0,1])}: w \in W^{m, \infty}([0,1]),\|w\|_{L^{\infty}([0,1])} \leq 1,\left\|w^{m}\right\|_{L^{\infty}([0,1])} \leq \sigma\right\}
$$

are explicitly known (at least for a wide range of parameters $m \in \mathbb{N}$ and $\sigma \geq 0$ ). The optimal Kolmogorov-Landau constants in these cases are given by the end-point values of certain Chebyshev type perfect splines. We refer to the papers by A. Pinkus [34] and S. KarLin [22], as well as the recent article by A. SHADRIN [35] and references therein.

\section{Appendix D. Proof of Lemma 1.1}

Proof. Let $f \in L_{2}^{1}\left(\mathbb{R}^{d}\right) \cap L \log L\left(\mathbb{R}^{d}\right)$ Then

$$
|H(f)|=\int_{\mathbb{R}^{d}} f \log _{+} f \mathrm{~d} v+\int_{\mathbb{R}^{d}} f \log _{-} f \mathrm{~d} v .
$$


The positive part is bounded by $\int f \log (1+f) \mathrm{d} v=\|f\|_{L \log L}$. The negative part can be controlled by

$$
\int_{\mathbb{R}^{d}} f \log _{-} f \mathrm{~d} v=\int_{\{f \leq 1\}} f \log \frac{1}{f} \mathrm{~d} v \leq C_{\delta} \int_{\{f \leq 1\}} f^{1-\delta} \mathrm{d} v \leq C_{\delta}\left(\int_{\mathbb{R}^{d}}\left(1+|v|^{2}\right)^{-\frac{1-\delta}{\delta}} \mathrm{d} v\right)^{\delta}\|f\|_{L_{2}^{1}}^{1-\delta}
$$

which is finite for $0<\delta<\frac{2}{d+2}$, having used that for any $\delta>0$ there exists a constant $C_{\delta}$ such that $\log t \leq C_{\delta} t^{\delta}$ for all $t \geq 1$.

Conversely, let $f \in L_{2}^{1}\left(\mathbb{R}^{d}\right)$ with finite entropy $H(f)$. Then

$$
\int_{\mathbb{R}^{d}} f \log (1+f) \mathrm{d} v=\int_{\{f \leq 1\}} f \log (1+f) \mathrm{d} v+\int_{\{f>1\}} f \log (1+f) \mathrm{d} v
$$

On where $f \leq 1$, we replace $f$ by 1 and where $f>1$, we bound $1+f$ by $2 f$ leading to

$$
\int_{\mathbb{R}^{d}} f \log (1+f) \mathrm{d} v \leq \log 2 \int_{\mathbb{R}^{d}} f \mathrm{~d} v+\int_{\mathbb{R}^{d}} f \log f \mathrm{~d} v+\int_{\mathbb{R}^{d}} f \log f \mathrm{~d} v
$$

As above, we conclude

$$
\int_{\mathbb{R}^{d}} f \log (1+f) \mathrm{d} v \leq \log 2\|f\|_{L^{1}\left(\mathbb{R}^{d}\right)}+H(f)+C_{\delta, d}\|f\|_{L_{2}^{1}\left(\mathbb{R}^{d}\right)^{1}}^{1-\delta} .
$$

with a finite constant $C_{\delta, d}$ for $0<\delta<\frac{2}{d+2}$.

Acknowledgements. We would like to thank Radjesvarane Alexandre for a discussion emphasising the question of smoothing properties of the Boltzmann equation. It is a pleasure to thank the REB program of CIRM for giving us the opportunity to start this research. J.-M. B. was partially supported by the project SQFT ANR-12-JS01-0008-01. D. H., T. R., and S. V. gratefully acknowledge financial support by the Deutsche Forschungsgemeinschaft (DFG) through CRC 1173. D. H. also thanks the Alfried Krupp von Bohlen und Halbach Foundation for financial support. Furthermore, we thank the University of Toulon and the Karlsruhe Institute of Technology for their hospitality.

\section{REFERENCES}

[1] R. Alexandre, Integral estimates for a linear singular operator linked with the Boltzmann operator. part I: Small singularities $0<v<1$, Indiana University Mathematics Journal 55 (2006), 1975-2022. http://dx.doi .org/10.1512/iumj .2006.55.2861.

[2] R. Alexandre, A review of Boltzmann equation with singular kernels, Kinetic and Related Models 2 (2009), 551646. http://dx.doi.org/10.3934/krm.2009.2.551.

[3] R. Alexandre, L. Desvillettes, C. Villani, and B. Wennierg, Entropy dissipation and long-range interactions, Archive for Rational Mechanics and Analysis 152 (2000), 327-355. http://dx. doi .org/10.1007/s002050000083.

[4] R. Alexandre and M. ElSAfadi, Littlewood-Paley theory and regularity issues in Boltzmann homogeneous equations. I. Non-cutoff case and Maxwellian molecules, Mathematical Models and Methods in Applied Sciences 15 (2005), 907-920. http://dx. doi.org/10.1142/S0218202505000613.

[5] R. Alexandre and L. He, Integral estimates for a linear singular operator linked with Boltzmann operators part ii: High singularities $1 \leq v<2$, Kinetic and Related Models 1 (2008), 491-513. http://dx. doi .org/10.3934/krm.2008.1.491.

[6] R. Alexandre, Y. Мorimoto, S. Ukai, C.-J. Xu, and T. Yang, Regularizing effect and local existence for the non-cutoff Boltzmann equation, Archive for Rational Mechanics and Analysis 198 (2010), 39-123. http://dx. doi .org/10.1007/s00205-010-0290-1.

[7] L. Arkeryd, On the Boltzmann equation, Archive for Rational Mechanics and Analysis 45 (1972), 1-16. http://dx. doi .org/10.1007/BF00253392.

[8] L. ARKerYd, Intermolecular forces of infinite range and the Boltzmann equation, Archive for Rational Mechanics and Analysis 77 (1981), 11-21. http://dx . doi .org/10.1007/BF00280403.

[9] A. V. Bobylev, Exact solutions of the nonlinear Boltzmann equation and the theory of relaxation of a Maxwellian gas, translated from Teoreticheskaya i Matematicheskaya Fizika 60 No. 2 (1984), 280-310, Theoretical and Mathematical Physics 60 (1984), 820-841. http://dx. doi .org/10 . 1007/BF01018983.

[10] L. Boltzmann, Weitere Studien über das Wärmegleichgewicht unter Gasmolekülen, Sitzungsberichte der Mathematisch-Naturwissenschaftlichen Classe der Kaiserlichen Akademie der Wissenschaften II. Abtheilung 66 (1872), 275-370. Available at http://dx . doi .org/10.1017/СВ09781139381420.023. 
[11] L. Desvillettes, About the regularizing properties of the non-cut-off Kac equation, Communications in Mathematical Physics 168 (1995), 417-440. http://dx. doi .org/10.1007/BF02101556.

[12] L. Desvillettes, Regularization properties of the 2-dimensional non radially symmetric non cutoff spatially homogeneous Boltzmann equation for Maxwellian molecules, Transport Theory and Statistical Physics 26 (1997), 341-357. http://dx. doi .org/10.1080/00411459708020291.

[13] L. Desvillettes, Boltzmann's kernel and the spatially homogeneous Boltzmann equation, Rivista di Matematica della Università di Parma (6) 4* (2001), 1-22. Available at http://www .rivmat. unipr.it/vols/2001-4s/indice.html.

[14] L. Desvillettes, About the use of Fourier transform for the Boltzmann equation, Rivista di Matematica della Università di Parma (7) $\mathbf{2}^{*}$ (2003), 1-99. Available at http://www.rivmat. unipr.it/vols/2003-2s/indice.html.

[15] L. Desvillettes, G. Furioli, and E. Terraneo, Propagation of gevrey regularity for solutions of the Boltzmann equation for Maxwellian molecules, Transactions of the American Mathematical Society 361 (2009), 1731-1747. http://dx. doi .org/10.1090/S0002-9947-08-04574-1.

[16] L. Desvillettes and B. Wennberg, Smoothness of the solution of the spatially homogeneous Boltzmann equation without cutoff, Communications in Partial Differential Equations 29 (2004), 133-155. http://dx.doi .org/10.1081/PDE-120028847.

[17] R. A. DeVore and G. G. Lorentz, Constructive Approximation, Grundlehren der mathematischen Wissenschaften 303, Springer-Verlag Berlin Heidelberg, 1993.

[18] W. Gautschi, On inverses of Vandermonde and confluent Vandermonde matrices, Numerische Mathematik 4 (1962), 117-123. http: //dx. doi .org/10.1007/BF01386302.

[19] L. Glangetas and M. NaJeme, Analytical regularizing effect for the radial and spatially homogeneous Boltzmann equation, Kinetic and Related Models 6 (2013), 407-427. http://dx . doi . org/10. 3934/krm. 2013 . 6. 407.

[20] T. Goudon, On Boltzmann equations and Fokker-Planck asymptotics: Influence of grazing collisions, Journal of Statistical Physics 89 (1997), 751-776. http://dx.doi.org/10.1007/BF02765543.

[21] M. Kac, Probability and Related Topics in Physical Sciences, 1 ed., Lectures in Applied Mathematics Vol. 1.A, Interscience Publishers, London, 1959.

[22] S. KaRLin, Interpolation properties of generalized perfect splines and the solutions of certain extremal problems. i, Transactions of the American Mathematical Society 206 (1975), 25-66. Available at http://www. jstor.org/stable/1997146.

[23] N. Lekrine and C.-J. Xu, Gevrey regularizing effect of the Cauchy problem for non-cutoff homogeneous Kac's equation, Kinetic and Related Models 2 (2009), 647-666. http://dx . doi . org/10. 3934/krm.2009.2 . 647.

[24] N. Lerner, Y. Morimoto, K. Pravda-Starov, and C.-J. Xu, Gelfand-Shilov smoothing properties of the radially symmetric spatially homogeneous Boltzmann equation without angular cutoff, Journal of Differential Equations 256 (2014), 797-831. http://dx. doi .org/10.1016/j.jde.2013.10.001.

[25] C. D. Levermore and M. Oliver, Analyticity of solutions for a generalized Euler equation, Journal of Differential Equations 133 (1997), 321-339. http://dx . doi . org/10.1006/jdeq. 1996. 3200.

[26] S.-Y. LiN, Gevrey regularity for the noncutoff nonlinear homogeneous boltzmann equation with strong singularity, Abstract and Applied Analysis (2014), Article ID 584169. http://dx . doi .org/10.1155/2014/584169.

[27] P.-L. Lions, Compactness in Boltzmann's equation via Fourier integral operators and applications. I, II, III, Journal of Mathematics of Kyoto University 34 (1994), 391-427, 429-461, 539-584.

[28] S. Mischler and B. Wennberg, On the spatially homogeneous Boltzmann equation, Annales de l'Institut Henri Poincare (C) Non Linear Analysis 16 (1999), 467-501. http://dx. doi . org/10 . 1016/s0294-1449(99) 80025-0.

[29] Y. Мовімото and S. UкаI, Gevrey smoothing effect of solutions for spatially homogeneous nonlinear Boltzmann equation without angular cutoff, Journal of Pseudo-Differential Operators and Applications 1 (2010), 139-159. http://dx. doi.org/10.1007/s11868-010-0008-z.

[30] Y. Morimoto, S. Ukai, C.-J. Xu, and T. YANG, Regularity of solutions to the spatially homogeneous Boltzmann equation without angular cutoff, Discrete and Continuous Dynamical Systems 24 (2009), 187-212. http://dx.doi.org/10.3934/dcds.2009.24.187.

[31] Y. Мовімото and C.-J. Xu, Ultra-analytic effect of Cauchy problem for a class of kinetic equations, Journal of Differential Equations 247 (2009), 596-617. http://dx. doi . org/10.1016/j . jde.2009.01.028.

[32] C. Моuнот and C. VILlani, Regularity theory for the spatially homogeneous boltzmann equation with cut-off, Archive for Rational Mechanics and Analysis 173 (2004), 169-212. http://dx. doi .org/10.1007/s00205-004-0316-7.

[33] Y.-P. PAO, Boltzmann collision operator with inverse-power intermolecular potentials, I, II, Communications on Pure and Applied Mathematics 27 (1974), 407-428, 559-581. http://dx.doi.org/10.1002/cpa.3160270402,10.1002/cpa.3160270406.

[34] A. Pinkus, Some extremal properties of perfect splines and the pointwise landau problem on the finite interval, Journal of Approximation Theory 23 (1978), 37-64.

[35] A. Shadrin, The Landau-Kolmogorov inequality revisited, Discrete and Continuous Dynamical Systems 34 (2014), 1183-1210. http://dx.doi.org/10.3934/dcds.2014.34.1183. 
[36] G. Toscani and C. Villani, Probability metrics and uniqueness of the solution to the Boltzmann equation for a Maxwell gas, Journal of Statistical Physics 94 (1999), 619-637. http: //dx . doi . org/10 . 1023/A : A1004508706950.

[37] C. Truesdell, On the pressures and the flux of energy in a gas according to Maxwell's kinetic theory, II, Journal of Rational Mechanics and Analysis 5 (1956), 55-128. Available at http: //wWW . iumj . indiana. edu/IUMJ/dfull text . php?year=1956\&volume=5\&artid=55002 .

[38] S. UKaI, Local solutions in Gevrey classes to the nonlinear Boltzmann equation without cutoff, Japan Journal of Applied Mathematics 1 (1984), 141-156. http://dx. doi .org/10.1007/BF03167864.

[39] C. Villani, On a new class of weak solutions to the spatially homogeneous Boltzmann and Landau equations, Archive for Rational Mechanics and Analysis 143 (1998), 273-307. http: //dx. doi .org/10.1007/s002050050106.

[40] C. Villani, A review of mathematical topics in collisional kinetic theory, in Handbook of Mathematical Fluid Dynamics (S. Friedlander and D. Serre, eds.), Vol. 1, Elsevier Science B.V., Amsterdam, 2002, pp. 71-305. http://dx.doi.org/10.1016/S1874-5792(02)80004-0.

[41] Z. Yin and T.-F. Zhang, Gevrey regularity for solutions of the non-cutoff Boltzmann equation: The spatially inhomogeneous case, Nonlinear Analysis: Real World Applications 15 (2014), 246-261. http://dx. doi .org/10.1016/j.nonrwa.2013.08.003.

[42] T.-F. Zhang and Z. YIN, Gevrey regularity of spatially homogeneous Boltzmann equation without cutoff, Journal of Differential Equations 253 (2012), 1172-1190. http: //dx . doi . org/10.1016/j . jde . 2012 . 04 .023.

\section{Jean-Marie Barbaroux}

Aix-Marseille Université, CNRS, CPT, UMR 7332, 13288 Marseille, France

et Université de Toulon, CNRS, CPT, UMR 7332, 83957 La Garde, France

E-mail: barbarou@univ-tln.fr

\section{Dirk Hundertmark}

Karlsruhe Institute of Technology, Englerstrasse 2, 76131 Karlsruhe, Germany

E-mail: dirk.hundertmark@kit.edu

\section{Tobias Ried}

Karlsruhe Institute of Technology, Englerstrasse 2, 76131 Karlsruhe, Germany

E-mail: tobias.ried@kit.edu

\section{Semjon Vugalter}

Karlsruhe Institute of Technology, Englerstrasse 2, 76131 Karlsruhe, Germany

E-mail: semjon.wugalter@kit.edu 